\title{
Fees, Reputation and Information Production in the Credit Rating Industry*
}

\author{
Jacopo Bizzotto ${ }^{\dagger}$ Adrien Vigier ${ }^{\ddagger}$
}

August 2019

\begin{abstract}
We compare a credit rating agency's incentives to acquire costly information when it is only paid for giving favorable ratings to the corresponding incentives when the agency is paid upfront, i.e. irrespective of the ratings assigned. We show that, in the presence of moral hazard, contingent fees provide stronger dynamic incentives to acquire information than upfront fees and may induce higher social welfare. When the fee structure is chosen by the agency, contingent fees arise as an equilibrium outcome, in line with the way the market for credit rating actually works.
\end{abstract}

JEL classification: D82, D83, G24.

Keywords: Credit Rating Agencies, Information Acquisition, Reputation.

\footnotetext{
*We thank Sambuddha Ghosh, Bård Harstad, Stefan Hirth, Steinar Holden, Albert Ma, Henry Mak, Kristoffer Midttømme, Tore Nilssen, Andrew Newman, Paolo Piacquadio, Francesco Sangiorgi, and various seminar audiences for helpful discussions.

†Oslo Metropolitan University. Email: jacopo.bizzotto@oslomet.no.

${ }_{\ddagger}^{\ddagger}$ BI Norwegian Business School. Email: a.h.vigier@gmail.com.
} 


\section{Introduction}

Credit rating agencies' (CRAs) principal source of revenue comes from issuers of rated securities, in the form of fees paid only if the issuer chooses to publish the rating obtained. ${ }^{1}$ To receive any fees, rating agencies are thus effectively forced to give favorable ratings. To tackle the resulting adverse selection problem, several commentators have proposed that issuers instead be required to pay CRAs upfront, that is, independently of whether or not an issuer received a favorable rating. ${ }^{2}$ However, the difficulty to monitor CRAs' research activities adds a moral hazard dimension to the problem of regulating the credit rating industry. As noted by a US Senate report, during the build-up of the financial crisis "neither Moody's nor S\&P hired sufficient staff or devoted sufficient resources to ensure that the initial rating process ... produced accurate credit ratings." 3 The primary goal of our paper is to argue that -in the presence of moral hazard- contingent fees provide stronger dynamic incentives to acquire information than upfront fees and to show that contingent fees can in fact result in more information acquisition and higher social welfare than upfront fees.

We study a CRA rating an infinite sequence of short-lived firms. Each firm seeks to finance a project with uncertain return. Costly information acquisition enables the agency to determine those returns. However, whether the agency shirks or acquires information is unobserved by firms and investors. Each period, the agency's choice is between (a) acquiring information to assign a favorable rating if and only if this period's project is high return, and (b) shirking and assigning a favorable rating automatically. In the spirit of Kreps and Wilson (1982) and Milgrom and Roberts (1982) the agency is one of two private types, "committed" or "strategic". The committed agency makes choice (a) in all periods. The strategic agency chooses between (a) and (b) with a view to maximize its expected intertemporal profit. The reputation of the agency refers to firms and investors' belief that the agency is committed. Each period, the reputation of the agency is updated based on the accuracy of the last period's rating.

We assume that firms pay fees proportional to the expected financial gain which a rating induces, and examine two settings. The contingent-fee setting is such that fees are only paid if and when a firm obtains a favorable rating. These fees are proportional to the financial gain resulting from a favorable rating. The upfront-fee setting is such that fees are paid before

\footnotetext{
${ }^{1}$ See, e.g., US SEC (2012): 12.

${ }^{2}$ Andrew Cuomo, who served as Attorney General of New York during the financial crisis, was among the first public figures to support this change of regulation.

${ }^{3}$ US Senate (2011): 304.
} 
ratings are assigned. These fees are thus proportional to the expected financial gain induced by the rating process. In either setting, the agency's choice of information acquisition is derived by weighing the immediate gains from shirking and assigning a favorable rating against future gains derived from a high reputation (obtained in turn through correct ratings). ${ }^{4}$ Compared to fees paid upfront, contingent fees raise the immediate gains from assigning a favorable rating. Moreover, this effect strengthens with the reputation of the agency (since increasing the agency's reputation pushes the fees upward). Hence, when the agency's reputation is initially high, upfront fees induce more information acquisition than contingent fees. ${ }^{5}$

On the other hand, when the agency's reputation is initially low then reputational incentives can induce the strategic agency to acquire more information under contingent fees than under upfront fees. The logic is the following. The more reputable the agency, the lower the chances that a firm will obtain a favorable rating from the agency. So fees paid upfront are in part pressed downward by an increase in the agency's reputation. This, in turn, implies that, from the viewpoint of the agency, incentives to build up reputation are weaker in the upfront-fee setting than in the contingent-fee setting. We show in our main theorem that these reputational incentives can be sufficient to assure more information acquisition and higher social welfare when fees are contingent than when they are upfront. This result suggests that replacing contingent fees with upfront fees could be socially damaging in markets where all CRAs have become suspect, as is perhaps the case today. The same would be true of markets with many new CRAs having to build up their reputations. ${ }^{6}$

In the second part of the paper we extend our model so as to explore the strategic agency's choice of fee structure. We allow the agency to make each period a take-it-or-leave-it offer to the firm it proposes to rate. We assume -in line with current practice in the industry- that the fee offered by the agency is unobserved by investors. First, contingent fees arise as an equilibrium outcome irrespective of the agency's reputation. This prediction matches the way the market for credit rating actually works. Second, in any equilibrium, the agency's takeit-or-leave-it offers induce the same amount of information acquisition as the contingent-fee structure examined in the first part our paper, irrespective of whether or not contingentfees are socially optimal. Our findings thus suggest that regulatory intervention might be necessary.

\footnotetext{
${ }^{4}$ In the model we assume that the cost of information is sufficiently small that acquiring information is socially optimal. So more information acquisition implies higher social welfare.

${ }^{5}$ This is the adverse selection effect we mentioned in the first paragraph of this introduction.

${ }^{6}$ We thank an anonymous referee for these observations.
} 
Our analysis and results have two main policy implications. We confirm first of all that if a regulator monitors information acquisition and sanctions shirking (thus eliminating moral hazard) then upfront fees are (a) socially optimal and (b) superior to contingent fees. The second half of our paper suggests an alternative policy: the regulator could leave information acquisition unchecked, but provide a way for CRAs to publicly commit to the fees they charge. This would solve the main friction currently causing CRAs to choose inefficient fees, namely the unobservability of CRAs' fee structure. As long as investors have sufficient knowledge of CRAs' strategic incentives, observable fees make it optimal for CRAs to choose an efficient fee structure. Lastly, note that while the focus of our paper is on the credit rating industry, our analysis applies more generally to any market comprising seller-paid ratings; markets with eco-labels for instance are prominent examples.

The paper is structured as follows. The related literature is discussed below. The model is presented in Section 2. An overview of the main result is given in Section 3, in a simplified two-period setting. The core analysis is in Section 4. Section 5 extends our baseline model by endogenizing the agency's fee structure. We discuss in Section 6 the limitations of our model. Section 7 concludes.

Related Literature. This paper contributes to the literature on credit rating by analyzing the link between different compensation schemes and a rating agency's incentives to acquire costly information. Specifically, we identify the conditions under which fees paid upfront by the issuer of a security generate less shirking than fees paid only in case of a favorable rating. Our paper builds on the work of Mathis, McAndrews and Rochet (2009) showing that when fees are contingent then reputational concerns are typically insufficient to discipline rating agencies. The effects of reputational concerns in the credit rating industry are further analyzed in five recent papers: Fulghieri, Strobl and Xia (2014), Frenkel (2015), Kashyap and Kovrijnykh (2016), Bouvard and Levy (2018) and Kovbasyuk (2018). Fulghieri et al. (2014) analyze the effect of introducing unsolicited credit ratings on CRAs' behavior in a model with contingent fees. The authors show that the adoption of unsolicited ratings raises CRAs' profits, and can lead to more informative ratings, thereby improving social welfare. Both Frenkel (2015) and Bouvard and Levy (2018) examine a rating agency paid upfront by the rated firms. In Frenkel (2015), the CRA has an incentive to maintain a reputation for credibility among investors but also an incentive to develop a second reputation for leniency among issuers. The author shows that in markets with few issuers, these incentives may lead the CRA to inflate ratings as a strategic tool to form a "double reputation". In the setting of Bouvard and Levy (2018), 
the agency chooses how much information to acquire. The firms' projects are heterogeneous in quality and so a reputation for providing accurate ratings attracts high-quality firms but repels low-quality firms. The agency thus aims for a balanced reputation. The fee structure of CRAs is endogenized in both Kashyap and Kovrijnykh (2016) and Kovbasyuk (2018). In Kashyap and Kovrijnykh (2016) the focus is on who should pay for the ratings. The authors find that rating errors tend to be larger in the issuer-pays models than in the investor-pays model. Kovbasyuk (2018) analyzes the merits of forcing CRAs to publish the fees charged to rated firms and shows that whereas private payments lead to coarse ratings, public payments on the other hand induce precise ratings at the top of the rating scale.

Our paper is related at a broader level to the vast literature on rating agencies recently surveyed in Jeon and Lovo (2013) and Sangiorgi and Spatt (2017), and particularly to two strand of papers, those studying information acquisition in the credit rating industry and those exploring rating inflation. Both Kashyap and Kovrijnykh (2016) and Bouvard and Levy (2018) belong to the first strand of research, which also includes Opp, Opp and Harris (2013) and Bongaerts (2014), though the focus of the latter papers bears little relation to what we do. $^{7}$ The strand of research exploring rating inflation includes Skreta and Veldkamp (2009) on the connection between asset complexity and rating inflation, Sangiorgi and Spatt (2016) examining the implications of opacity about contacts between issuers and rating agencies, and Goldstein and Huang (2017) on the link between rating inflation and firms' investments. Bolton, Freixas and Shapiro (2012) note for instance that upfront fees eliminate the incentives for CRAs to inflate ratings, but do not eliminate shopping.

Finally, we are connected more generally to the literature exploring the regulation of markets for financial advice surveyed in Inderst and Ottaviani (2012), and to the work of Chade and Kovrijnykh (2016) exploring optimal contracts for delegated information acquisition. However, since we aim to capture frictions that are specific to the market for credit ratings, the settings of those papers differ in many ways from ours.

\section{A Model of Credit Rating with Contingent Fees}

We examine a CRA rating a sequence of short-lived firms indexed by $t \in\{1,2, \ldots\}$. Each firm seeks to persuade investors to finance a project with uncertain (net) return $q_{t} \in\{-1,1\}$. The

\footnotetext{
${ }^{7}$ Opp et al. (2013) examine the impact of rating-contingent regulations. Bongaerts (2014) sheds light on the relative merits of the investor-pays vs issuer-pays models of the rating industry by investigating which alternative generates more shirking.
} 
sequence $\left\{q_{t}\right\}$ is independent and identically distributed according to $\mathbb{P}\left(q_{t}=1\right)=\frac{1}{2} \cdot{ }^{8}$ Firms which obtain a favorable rating pay to the agency a fee proportional to investors' perceived increase in their project's expected return. Incurring a cost enables the agency to learn a project's return, but information acquisition is unobservable. The agency is either committed to acquire information in all periods, or behaves strategically so as to maximize its expected intertemporal profit. The details of the model are laid out below. We shall at times refer to this model as the contingent-fee setting (the upfront-fee setting will be presented in Section 4).

Ratings and Information Acquisition. Each period the agency assigns a rating $r_{t} \in$ $\{-1,1\}$ (potentially) informing firms and investors about $q_{t}$. Incurring a cost $c \in\left(0, \frac{1}{2}\right)$ at the beginning of period $t$ enables the agency to observe $q_{t}$ (noisy signals of project quality are considered in Online Appendix A). Assuming $c<\frac{1}{2}$ ensures that acquiring information is socially optimal. The choice of the agency is between (a) acquiring information to assign $r_{t}=q_{t}$ and (b) automatically assigning $r_{t}=1$ without acquiring information in the first place. ${ }^{9}$ Information acquisition is unobservable and therefore non-contractible.

Reputation. The agency is of one of two types: committed or strategic. The agency's type is private information, and determined by nature once and for all at the onset of the game. Each period the committed agency acquires information and assigns $r_{t}=q_{t}$. The strategic agency on the other hand chooses between acquiring information and shirking with a view to maximize profits over the infinite horizon. Let $\rho_{t}$ denote firms and investors' beginning-ofperiod- $t$ belief that the agency is committed. We assume $\rho_{1} \in(0,1)$ and refer to $\rho_{t}$ as the agency's reputation in period $t$.

Firms and Investors. At the beginning of each period firms and investors form beliefs regarding the probability that the strategic agency will acquire information in the current period; let $\hat{e}_{t}$ denote the probability attached to the strategic agency acquiring information in period $t$.

If the agency announces $r_{t}=-1$ then firm $t$ 's project is instantly dropped. On the other hand, by Bayes' rule, the rating $r_{t}=1$ raises firms and investors' perceived expected return

\footnotetext{
${ }^{8}$ We abuse notation slightly and use $q_{t}$ to denote both the random variable and its realization.

${ }^{9}$ That the agency (when it acquires information) truthfully reports what it observes is without loss of generality, as shirking always dominates acquiring information followed by misreporting $q_{t}$. The case in which the agency possibly deflates ratings when shirking is considered in Online Appendix B.
} 
from 0 to

$$
\mathbb{E}\left[q_{t} \mid r_{t}=1, \rho_{t}, \hat{e}_{t}\right]=\frac{1-\left(1-\rho_{t}\right)\left(1-\hat{e}_{t}\right)}{1+\left(1-\rho_{t}\right)\left(1-\hat{e}_{t}\right)} .
$$

Investors are assumed to be on the long side of the market, so that if $r_{t}=1$, the project of firm $t$ is sold to them at the price $\mathbb{E}\left[q_{t} \mid r_{t}=1, \rho_{t}, \hat{e}_{t}\right] \geq 0$.

Fees. The agency obtains a fraction $\beta$ of all proceeds resulting from selling projects to investors. For now $\beta$ is exogenous and can be interpreted as the bargaining power of the agency (Section 5 endogenizes the fee structure). The agency's fee $\phi_{t}\left(r_{t}\right)$, which depends on the rating assigned, is thus given by ${ }^{10}$

$$
\phi_{t}\left(r_{t}\right)= \begin{cases}\beta \mathbb{E}\left[q_{t} \mid r_{t}=1, \rho_{t}, \hat{e}_{t}\right] & \text { if } r_{t}=1 \\ 0 & \text { if } r_{t}=-1\end{cases}
$$

If $\beta<2 c$ then, in the upfront-fee setting presented in Section 4 , the strategic agency always shirks. To make the analysis interesting we thus assume $\beta>2 c$.

Timing. The timing within a period is as follows (see Figure 1). The agency first decides whether or not to acquire information. Based on information gathered (if any), the agency publicly announces $r_{t}$, and receives the fee $\phi_{t}\left(r_{t}\right)$. All players observe $q_{t}$ and the game moves on to the next period. ${ }^{11}$

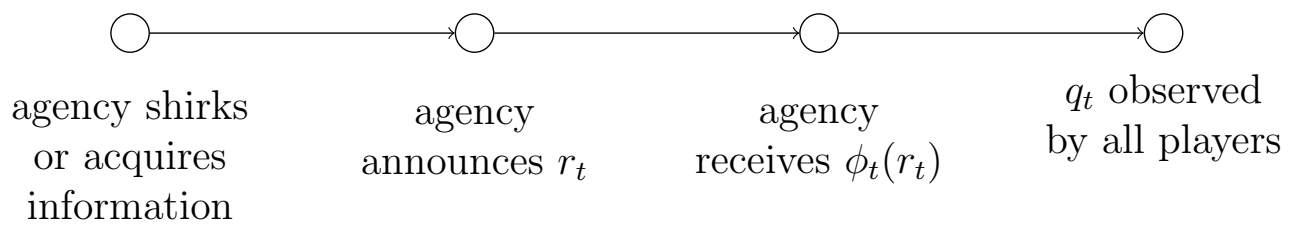

FigURE 1: TIMELINE

Strategies and Payoffs. The period- $t$ profit of the agency is $\pi_{t}:=\phi_{t}\left(r_{t}\right)-c \mathbf{1}_{\{\text {info. acq. in period } t\}}$, where $\mathbf{1}_{X}$ denotes the indicator function of $X$. The payoff of firm $t$ is $\mathbf{1}_{\left\{r_{t}=1\right\}} \mathbb{E}\left[q_{t} \mid r_{t}=\right.$ $\left.1, \rho_{t}, \hat{e}_{t},\right]-\phi_{t}\left(r_{t}\right)$. Finally, investors' payoff in period $t$ is given by $\mathbf{1}_{\left\{r_{t}=1\right\}}\left(q_{t}-\mathbb{E}\left[q_{t} \mid r_{t}=1, \rho_{t}, \hat{e}_{t}\right]\right)$.

\footnotetext{
${ }^{10}$ All our results carry through more generally as long as the fee is some increasing function of all proceeds resulting from selling projects to investors. See Online Appendix C.

${ }^{11}$ Whether or not $q_{t}$ is observed when $r_{t}=-1$ is irrelevant, since $r_{t}=-1$ implies $q_{t}=-1$. Assuming that $q_{t}$ is always observed at the end of a period enables us to economize on notation.
} 
All payoffs are discounted according to the discount factor $\delta \in(0,1), \delta \neq \frac{2 \beta+4 c}{3 \beta+2 c}$. ${ }^{12}$ We refer to $\sum_{t=1}^{\infty} \delta^{t-1} \pi_{t}$ as the agency's intertemporal profit. Social welfare, $W$, is defined as the discounted sum of all players' payoffs:

$$
W:=\sum_{t=1}^{\infty} \delta^{t-1}\left(q_{t} \mathbf{1}_{\left\{r_{t}=1\right\}}-c \mathbf{1}_{\{\text {information acquired in period } t\}}\right)
$$

As $c<\frac{1}{2}$, acquiring information each period maximizes expected social welfare.

Equilibrium. A strategy of the agency specifies a probability of acquiring information as a function of the history. ${ }^{13}$ We focus on Perfect Bayesian Equilibria in which the strategic agency uses a stationary Markov strategy with reputation as the state variable. Let

$$
\rho_{t}^{+}:=\frac{\rho_{t}}{\rho_{t}+\left(1-\rho_{t}\right) \hat{e}_{t}}
$$

The following definition is adapted from Mathis et al. (2009):

Definition 1. An equilibrium is a mapping $e:[0,1] \rightarrow[0,1]$ specifying the probability $e\left(\rho_{t}\right)$ that the strategic agency acquires information given reputation $\rho_{t}$, such that, for all $\rho_{1}$ :

(i) the strategy $e(\cdot)$ maximizes the agency's expected intertemporal profit given $\rho_{t+1}=\Psi\left(\rho_{t}, r_{t}, q_{t}\right)$, where

$$
\Psi\left(\rho_{t}, r_{t}, q_{t}\right):= \begin{cases}\rho_{t} & \text { if } q_{t}=1 \\ \rho_{t}^{+} & \text {if } q_{t}=-1=r_{t} \text { and } \rho_{t}>0 \\ 0 & \text { if } q_{t}=-1=-r_{t}, \text { or } \rho_{t}=0\end{cases}
$$

(ii) firms and investors' beliefs satisfy $\hat{e}_{t}=e\left(\rho_{t}\right)$.

In equilibrium investors correctly infer the probability with which the strategic agency chooses to acquire information, and the agency's choice of information acquisition is optimal given the evolution of beliefs captured by $\Psi$. The beliefs are updated using Bayes' rule whenever possible. In particular, if $q_{t}=1$ then $r_{t}=1$ whether the agency shirks or acquires

\footnotetext{
${ }^{12}$ We rule out $\delta=\frac{2 \beta+4 c}{3 \beta+2 c}$ for expositional simplicity. In this case, multiple equilibria exist which only differ off equilibrium path.

${ }^{13}$ The structure of the model enables us to focus on the single-agent decision problem facing the agency. Specifically, we simplified the exposition by leaving a number of "actions" outside of the model: we assumed that (a) conditional on $r_{t}=1$, firm $t$ sells the project to investors at the price $\mathbb{E}\left[q_{t} \mid r_{t}=1, \rho_{t}, \hat{e}_{t}\right]$, and (b) the division of surplus between the firms and the agency is determined by the exogenous parameter $\beta$.
} 
information, hence reputation does not change. If $q_{t}=-1$, two cases arise: $r_{t}=1$ reveals that the agency has shirked (and thus, that the agency is strategic), and $r_{t}=-1$ reveals that the agency has acquired information. In the latter case reputation (weakly) increases since the committed agency acquires information with probability 1. Zero-probability events are dealt with by assuring that $\rho_{t}=0$ is an absorbing state of the Markov process and by ascribing any misreporting to the strategic agency (that is, whether or not the initial probability that the agency is strategic is positive).

\section{Overview of the Main Result}

In this section we briefly analyze a two-period version of the model in order to develop intuition for the paper's main result.

We solve this game by backward induction. With two periods only, the strategic agency shirks at $t=2$. Then $\hat{e}_{2}=0$ and, by (1) and (2), the agency's period-2 profit is

$$
\pi_{2}^{c}\left(\rho_{2}\right)=\phi_{2}(1)=\beta \mathbb{E}\left[q_{2} \mid r_{2}=1, \rho_{2}, 0\right]=\frac{\beta \rho_{2}}{2-\rho_{2}}
$$

Consider now the agency's problem at $t=1$. If it shirks (and automatically assigns $r_{1}=1$ ) the agency's expected intertemporal profit is ${ }^{14}$

$$
\phi_{1}(1)+\delta\left(\frac{1}{2} \pi_{2}^{c}\left(\rho_{1}\right)+\frac{1}{2} \pi_{2}^{c}(0)\right) .
$$

If instead the agency acquires information, then its expected intertemporal profit is ${ }^{15}$

$$
\frac{1}{2} \phi_{1}(1)-c+\delta\left(\frac{1}{2} \pi_{2}^{c}\left(\rho_{1}\right)+\frac{1}{2} \pi_{2}^{c}\left(\rho_{1}^{+}\right)\right)
$$

where, recall, $\rho_{1}^{+}=\frac{\rho_{1}}{\rho_{1}+\left(1-\rho_{1}\right) \hat{e}_{1}}$ denotes the Bayes-updated belief that the agency is committed after observing that $q_{1}=-1=r_{1}$. Shirking is thus optimal if and only if

$$
\frac{1}{2} \phi_{1}(1)+c \geq \frac{\delta}{2}\left(\pi_{2}^{c}\left(\rho_{1}^{+}\right)-\pi_{2}^{c}(0)\right) .
$$

\footnotetext{
${ }^{14}$ To understand (3) note that with probability $\frac{1}{2}, q_{1}=1$ in which case nothing is learned about the type of the agency between periods 1 and $2\left(\rho_{2}=\rho_{1}\right)$, while with probability $\frac{1}{2}, q_{1}=-1$ in which case firms and investors observe that the agency shirked, and thus learn that the agency is strategic $\left(\rho_{2}=0\right)$.

${ }^{15}$ In this case the agency receives the fee $\phi_{1}(1)$ with probability $\frac{1}{2}$ only, since if $q_{t}=-1$ the agency announces $r_{t}=-1$ and receives $\phi_{1}(-1)=0$.
} 
The left-hand side represents the short-run incentive to shirk: $\frac{1}{2} \phi_{1}(1)$ captures the gain from securing a positive fee irrespective of period-1 project's return, and $c$ the saving from not paying the cost of information. The right-hand side represents the long-run incentive to acquire information, and captures the gain from obtaining a larger fee at $t=2$ due to a higher reputation.

In particular, an equilibrium in which the strategic agency shirks with probability 1 at $t=1$ exists if and only if (3) holds for $\hat{e}_{1}=0$. Noting that $\hat{e}_{1}=0$ implies $\rho_{1}^{+}=1$, straightforward algebra shows that this condition becomes

$$
\delta \leq \frac{\rho_{1}}{2-\rho_{1}}+\frac{2 c}{\beta}
$$

We next carry out similar calculations, but in a setting in which fees are paid upfront rather than depending on the rating assigned. We first note that, prior to knowing the rating assigned, firm $t$ 's "expected value of the rating $r_{t}$ " is $\mathbb{P}\left(r_{t}=1 \mid \rho_{t}, \hat{e}_{t}\right) \mathbb{E}\left[q_{t} \mid r_{t}=1, \rho_{t}, \hat{e}_{t}\right]$ (this is firm $t$ 's expected revenue from investors). So with fees paid upfront the fees received by the agency are

$$
\phi_{t}(1)=\phi_{t}(-1)=\beta \mathbb{P}\left(r_{t}=1 \mid \rho_{t}, \hat{e}_{t}\right) \mathbb{E}\left[q_{t} \mid r_{t}=1, \rho_{t}, \hat{e}_{t}\right]
$$

instead of (2). Then the agency (which still chooses to shirk at $t=2$ ) obtains the period-2 profit

$$
\pi_{2}^{u}\left(\rho_{2}\right)=\phi_{2}(1)=\beta \mathbb{P}\left(r_{2}=1 \mid \rho_{2}, 0\right) \mathbb{E}\left[q_{2} \mid r_{2}=1, \rho_{2}, 0\right]=\frac{\beta \rho_{2}}{2} .
$$

Consider now the agency's problem at $t=1$ in the upfront-fee setting. If it shirks the agency's expected intertemporal profit is

$$
\phi_{1}(1)+\delta\left(\frac{1}{2} \pi_{2}^{u}\left(\rho_{1}\right)+\frac{1}{2} \pi_{2}^{u}(0)\right)
$$

If instead the agency acquires information then its expected intertemporal profit is

$$
\phi_{1}(1)-c+\delta\left(\frac{1}{2} \pi_{2}^{u}\left(\rho_{1}\right)+\frac{1}{2} \pi_{2}^{u}\left(\rho_{1}^{+}\right)\right)
$$

Hence, here, shirking is optimal if and only if

$$
c>\frac{\delta}{2}\left(\pi_{2}^{u}\left(\rho_{1}^{+}\right)-\pi_{2}^{u}(0)\right)
$$

and an equilibrium in which the strategic agency shirks with probability 1 exists if and only 
if (5) holds for $\hat{e}_{1}=0$, giving, after a few steps of algebra,

$$
\delta \leq \frac{4 c}{\beta}
$$

Now notice that if $\rho_{1}$ is sufficiently small $\left(\rho_{1}<\frac{4 c}{\beta+2 c}\right) \beta, c$ and $\delta$ can be chosen such that $(\star \star)$ holds while $(\star)$ is violated. This shows that under certain conditions the strategic agency acquires information (with some positive probability) when fees are contingent but shirks when fees are paid upfront. ${ }^{16}$

To gain intuition for this finding, consider the marginal benefit from building a reputation in the contingent-fee setting $\left(\frac{d \pi_{2}^{c}}{d \rho_{2}}\right)$ and the corresponding benefit under upfront fees $\left(\frac{d \pi_{2}^{u}}{d \rho_{2}}\right)$. For all $\rho_{2}>0$ :

$$
\frac{d \pi_{2}^{c}}{d \rho_{2}}=\frac{2 \beta}{\left(2-\rho_{2}\right)^{2}}>\frac{\beta}{2}=\frac{d \pi_{2}^{u}}{d \rho_{2}}
$$

Consequently, the long-run incentive to acquire information is stronger under contingent than under upfront fees:

$$
\frac{\delta}{2}\left(\pi_{2}^{u}\left(\rho_{1}^{+}\right)-\pi_{2}^{u}(0)\right)>\frac{\delta}{2}\left(\pi_{2}^{c}\left(\rho_{1}^{+}\right)-\pi_{2}^{c}(0)\right), \quad \forall \hat{e}_{1} \in[0,1]
$$

The reason is that with upfront fees the agency is paid at $t=2$ proportionately to firm 2's belief that it will obtain a favorable rating (that is, proportionately to $\mathbb{P}\left(r_{2}=1 \mid \rho_{2}, 0\right)$ ). Yet, firm 2 expects a reputable agency to act truthfully, and thus to deliver negative ratings with high probability:

$$
\mathbb{P}\left(r_{2}=1 \mid \rho_{2}, 0\right)=\frac{1}{2} \cdot \rho_{2}+1 \cdot\left(1-\rho_{2}\right)
$$

is decreasing in $\rho_{2}$. So, in an upfront-fee setting, building a reputation is only moderately rewarding for the agency. By contrast, firm 2's belief that it will obtain a favorable rating plays no role under contingent fees, as the firm only pays the agency conditional on $r_{2}=1$.

As we will see in the next section, the previous findings do not rest on the two-period nature of the simple model examined here. We further show in the online appendices that these findings are robust to various modifications of the baseline model: we show in Online Appendix A that they still hold if the agency observes noisy signals of project quality; Online Appendix B checks the robustness of our analysis by allowing the strategic agency to announce $r_{t}=-1$ whether or not in period $t$ the agency chooses to acquire information; in Online

\footnotetext{
${ }^{16}$ This remark implicitly assumes that an equilibrium exists in the contingent-fee setting for the parameters we are considering. The existence of an equilibrium is easy to prove.
} 
Appendix $\mathrm{C}$ we show that the main results carry through if instead of being paid a fixed fraction $\beta$ of all proceeds from selling the projects to investors, the agency is paid an arbitrary increasing function of these proceeds; Online Appendix D allows the prior probability of $q_{t}=1$ to take any value in $(0,1)$; finally, Online Appendix E shows that our main results continue to hold in a setting in which $q_{t}$ is never observed by firms and investors.

\section{Main Analysis}

We characterize in Subsection 4.1 the unique equilibrium of the contingent-fee setting presented in Section 2. The upfront-fee setting is presented and examined in Subsection 4.2. Our first main result (Theorem 1) is stated and discussed in Subsection 4.3.

\subsection{Contingent Fees}

By Bellman's Principle of Optimality, an equilibrium with value function $V_{c}(\cdot)$ satisfies the equation

$V_{c}(\rho)=\max _{e \in[0,1]}\left\{(1-e)\left[\varphi_{c}(\rho)+\delta\left(\frac{1}{2} V_{c}(\rho)+\frac{1}{2} V_{c}(0)\right)\right]+e\left[\frac{\varphi_{c}(\rho)}{2}-c+\delta\left(\frac{1}{2} V_{c}(\rho)+\frac{1}{2} V_{c}\left(\rho^{+}\right)\right)\right]\right\}$,

where $\varphi_{c}\left(\rho_{t}\right):=\beta \mathbb{E}\left[q_{t} \mid r_{t}=1, \rho_{t}, e\left(\rho_{t}\right)\right]$ denotes the fee paid to the agency in case $r_{t}=1$ as a function of the agency's reputation $\rho_{t}$. As the maximand is linear in the control variable, the previous equation simplifies to

$$
V_{c}(\rho)=\max \left\{\varphi_{c}(\rho)+\delta\left(\frac{1}{2} V_{c}(\rho)+\frac{1}{2} V_{c}(0)\right), \frac{\varphi_{c}(\rho)}{2}-c+\delta\left(\frac{1}{2} V_{c}(\rho)+\frac{1}{2} V_{c}\left(\rho^{+}\right)\right)\right\}
$$

We show in the appendix that $V_{c}(0)=0$. Hence, if $\frac{\varphi_{c}(\rho)}{2}+c=\frac{\delta}{2} V_{c}\left(\rho^{+}\right)$the strategic agency is indifferent between shirking and acquiring information; if $\frac{\varphi_{c}(\rho)}{2}+c>\frac{\delta}{2} V_{c}\left(\rho^{+}\right)$shirking is uniquely optimal; and if $\frac{\varphi_{c}(\rho)}{2}+c<\frac{\delta}{2} V_{c}\left(\rho^{+}\right)$then acquiring information is uniquely optimal. In the terminology of Section $3, \frac{\varphi_{c}(\rho)}{2}+c$ represents the short-run incentive to shirk, while $\frac{\delta}{2} V_{c}\left(\rho^{+}\right)$represents the long run incentive to acquire information.

The following proposition establishes that an equilibrium exists, is unique, and exhibits a familiar pattern (Benabou and Laroque (1992), Mathis et al. (2009), Board and Meyerter Vehn (2013)): the strategic agency builds up reputation when it is low, and milks its reputation when it is high. 


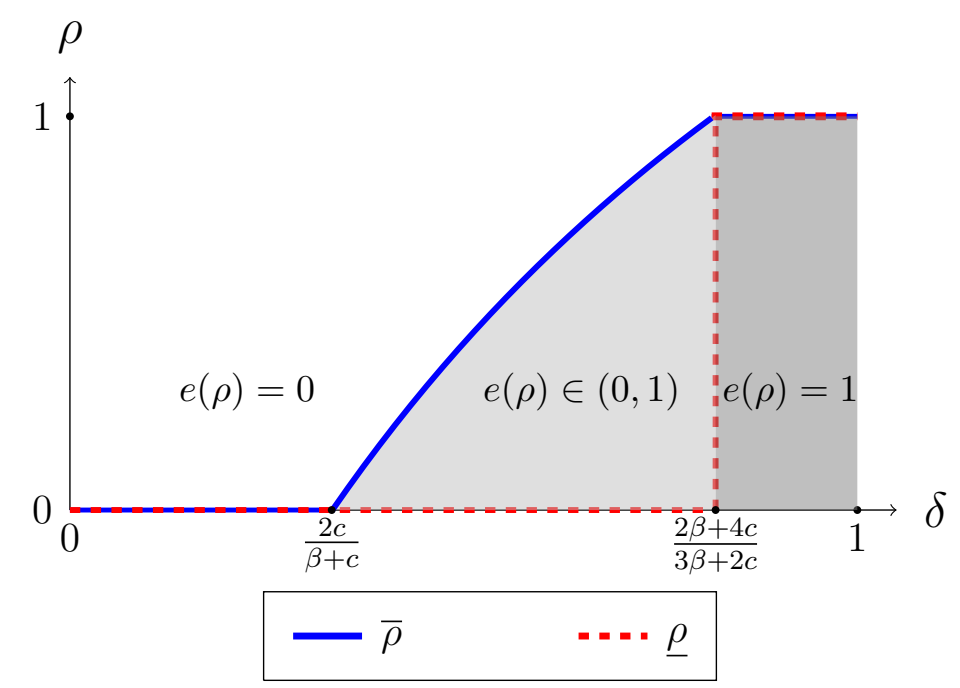

Figure 2: EQUiLIBRIUM With CONTINGENT FEeS

Proposition 1. An equilibrium of the contingent-fee setting exists and is unique. In equilibrium, $e(0)=0$; for $\rho>0$ the equilibrium is characterized by cutoffs $\underline{\rho}$ and $\bar{\rho}, \underline{\rho} \leq \bar{\rho}$, such that

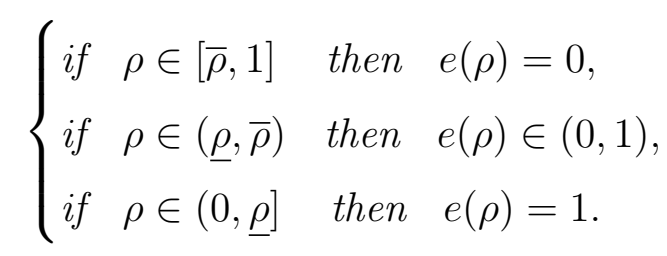

Moreover, in equilibrium, the fee $\phi_{t}(1)$ paid to the agency in the case of a favorable rating is a non-decreasing function of the agency's reputation $\rho_{t}$.

The proof of the proposition is in Appendix A. A description of the strategic agency's equilibrium behavior for different values of $\delta$ is given in Figure 2 (the details of all calculations are in Appendix A). For $\delta>\frac{2 \beta+4 c}{3 \beta+2 c}$ we obtain $\underline{\rho}=1$, that is, the strategic agency acquires information with probability 1 as long as its reputation is not zero. For $\delta<\frac{2 c}{\beta+c}$ we obtain $\bar{\rho}=0$, that is, the strategic agency shirks with probability 1 irrespective of its reputation. For $\delta \in\left(\frac{2 c}{\beta+c}, \frac{2 \beta+4 c}{3 \beta+2 c}\right)$ we obtain $\underline{\rho}=0<\bar{\rho}<1$, that is, the strategic agency shirks above a certain level of reputation, and randomizes between shirking and acquiring information below this reputation. 


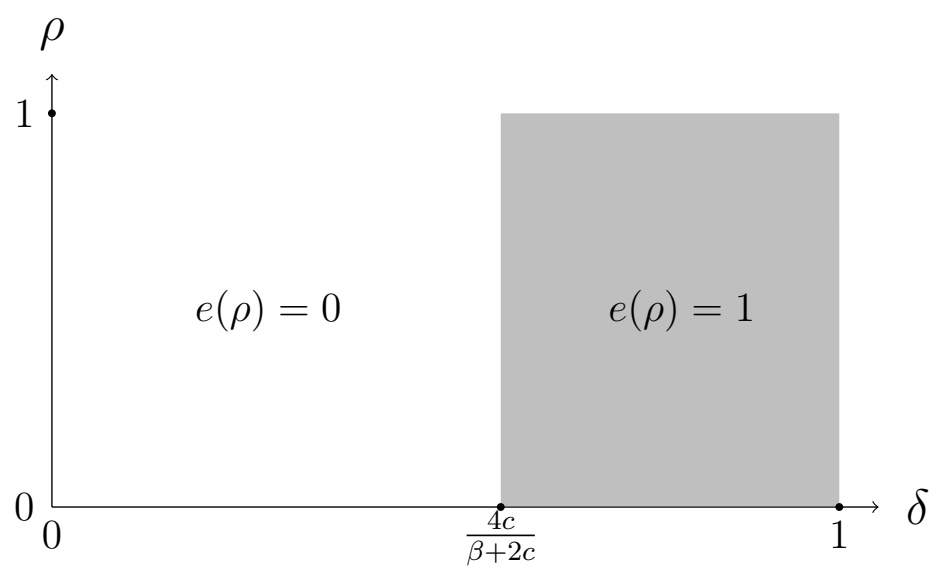

Figure 3: EQUILIBRIUM WITH UPFRONT FEES

\subsection{Upfront Fees}

In this subsection we present and analyze the upfront-fee setting. This setting replicates all features of the contingent-fee setting (see Section 2) except for the fact that since a firm's expected revenue from investors at the time of paying the fee is now $\mathbb{P}\left(r_{t}=1 \mid \rho_{t}, \hat{e}_{t}\right) \mathbb{E}\left[q_{t} \mid r_{t}=\right.$ $\left.1, \rho_{t}, \hat{e}_{t}\right]$, then (2) is accordingly replaced by (4).

We proceed to characterize the equilibrium behavior of the strategic agency in this setting. In equilibrium the agency is paid $\varphi_{u}\left(\rho_{t}\right):=\beta \mathbb{P}\left(r_{t}=1 \mid \rho_{t}, e\left(\rho_{t}\right)\right) \mathbb{E}\left[q_{t} \mid r_{t}=1, \rho_{t}, e\left(\rho_{t}\right)\right]$ in period $t$, and an equilibrium with value function $V_{u}(\cdot)$ satisfies the Bellman equation ${ }^{17}$

$$
V_{u}(\rho)=\max \left\{\varphi_{u}(\rho)+\delta\left(\frac{1}{2} V_{u}(\rho)+\frac{1}{2} V_{u}(0)\right), \varphi_{u}(\rho)-c+\delta\left(\frac{1}{2} V_{u}(\rho)+\frac{1}{2} V_{u}\left(\rho^{+}\right)\right)\right\} .
$$

We show in the appendix that $V_{u}(0)=0$. Hence, if $c=\frac{\delta}{2} V_{u}\left(\rho^{+}\right)$the strategic agency is indifferent between shirking and acquiring information; if $c>\frac{\delta}{2} V_{u}\left(\rho^{+}\right)$shirking is uniquely optimal; and if $c<\frac{\delta}{2} V_{u}\left(\rho^{+}\right)$acquiring information is then uniquely optimal. In other words, in this setting $c$ represents the short-run incentive to shirk, while $\frac{\delta}{2} V_{u}\left(\rho^{+}\right)$represents the long-run incentive to acquire information.

The following proposition establishes that an equilibrium exists and is unique.

${ }^{17}$ Where, as in Section 4.1, the equation in the text is obtained from

$$
V_{u}(\rho)=\max _{e \in[0,1]}\left\{(1-e)\left[\varphi_{u}(\rho)+\delta\left(\frac{1}{2} V_{u}(\rho)+\frac{1}{2} V_{u}(0)\right)\right]+e\left[\varphi_{u}(\rho)-c+\delta\left(\frac{1}{2} V_{u}(\rho)+\frac{1}{2} V_{u}\left(\rho^{+}\right)\right)\right]\right\}
$$

noting that the maximand is linear in the control variable. 
Proposition 2. If $\delta<\frac{4 c}{\beta+2 c}$ then $e(\rho)=0$ for all $\rho \in[0,1]$ is the unique equilibrium with upfront fees. If $\delta>\frac{4 c}{\beta+2 c}$ the unique equilibrium is

$$
e(\rho)=\left\{\begin{array}{l}
1 \text { if } \rho>0 \\
0 \text { if } \rho=0
\end{array}\right.
$$

Figure 3 illustrates the proposition. Its proof is in Appendix B. The familiar pattern of Proposition 1 is here replaced by a different one: either the strategic agency shirks irrespective of its reputation, or the strategic agency acquires information with probability 1 at all positive values of reputation. The difference between these patterns can be traced back to the relation between reputation and the short-run incentive to shirk in the two settings. With contingent fees, the short-run incentive to shirk is an increasing function of reputation. By contrast, with upfront fees the short-run incentive to shirk is independent of reputation.

\subsection{Main Result}

We are now ready to state our first main result: as acquiring information is socially optimal and $\frac{4 c}{\beta+2 c} \in\left(\frac{2 c}{\beta+c}, \frac{2 \beta+4 c}{3 \beta+2 c}\right)$, the combination of Propositions 1 and 2 yields the following theorem.

Theorem 1. There exists $\tilde{\rho}>0$ such that, if $\delta \in\left(\frac{2 c}{\beta+c}, \frac{4 c}{\beta+2 c}\right)$ then, for $\rho_{1} \in(0, \tilde{\rho})$, contingent fees improve expected social welfare relative to upfront fees. If instead $\delta \in\left(\frac{4 c}{\beta+2 c}, \frac{2 \beta+4 c}{3 \beta+2 c}\right)$, then upfront fees improve expected social welfare relative to contingent fees. In all other cases, expected social welfare is the same whether fees are upfront or contingent.

The first part of the theorem is our first main result: contingent fees can improve expected social welfare relative to upfront fees. The basic mechanism is as indicated in Section 3 for the two-period case, namely, contingent fees result in more information acquisition than upfront fees by inducing stronger long-run incentives to acquire information. To illustrate here this point in a simple way, it is useful to compare the long-run incentives to acquire information (that is, $\frac{\delta}{2} V_{i}(\cdot)$, for $i=c, u$ ) for the parametric region $\delta<\frac{2 c}{\beta+c}$ in which, under either fee setting, $e(\cdot)=0$ in equilibrium. Then the respective Bellman equations yield:

$$
V_{i}\left(\rho_{t}\right)=\frac{\varphi_{i}\left(\rho_{t}\right)}{1-\frac{\delta}{2}}, \quad \text { for } i=c, u
$$


We thus obtain first

$$
V_{u}\left(\rho_{t}\right)=\frac{\mathbb{P}\left(r_{t}=1 \mid \rho_{t}, 0\right) \varphi_{c}\left(\rho_{t}\right)}{1-\frac{\delta}{2}}=\mathbb{P}\left(r_{t}=1 \mid \rho_{t}, 0\right) V_{c}\left(\rho_{t}\right),
$$

followed by

$$
\frac{\delta}{2} V_{c}\left(\rho_{t}\right) \geq \frac{\delta}{2} V_{u}\left(\rho_{t}\right), \quad \forall \rho_{t}
$$

The agency's costly and unobservable information acquisition is the key friction underlying Theorem 1. To bring home this important point, recall that with upfront fees the short-run incentive to shirk is c only, hence, in the upfront-fee setting, for $c=0$ the strategic agency acquires information with probability 1 each period. ${ }^{18}$ Yet, by rewarding the agency for giving out favorable ratings, contingent fees incentivize rating inflation, irrespective of the cost of information. $^{19}$ In the absence of moral hazard upfront fees are thus socially optimal and provide unambiguously better incentives than contingent fees.

\section{$5 \quad$ Endogenous Fees}

We assumed up to this point that the fee structure of the rating agency was exogenously given. In this section we endogenize the fee structure and show that endogenous fees arise as an equilibrium outcome irrespective of the agency's reputation. While other fee structures may be possible, any of them induces the same amount of information acquisition as contingent fees (whether or not contingent fees are socially efficient). The key friction underlying this inefficiency is the non-observability by investors of the contracts offered by the agency.

We show first that (socially optimal) fee structures exist that induce the strategic agency to acquire information with probability 1 each period. To see this, consider the following family of fee structures: if $\rho_{t}=0$ then $\phi_{t}(1)=\phi_{t}(-1)=0$, while if $\rho_{t}=\rho_{1}$ then $\phi_{t}(1)=a$ and $\phi_{t}(-1)=b$. The necessary and sufficient conditions for such a fee structure to induce $e_{t}=1$ each period are: ${ }^{20}$

$$
\frac{b-a}{2}+\frac{a \delta}{2-\delta} \geq c
$$

and

$$
a+b \leq 1
$$

\footnotetext{
${ }^{18}$ Note that the threshold in Proposition $2, \frac{4 c}{\beta+2 c}$, is equal to 0 for $c=0$.

${ }^{19}$ Note that $\lim _{\delta \rightarrow 0} \underline{\rho}=0$ for all values of $c$ (see the proof of Proposition 1 ).

${ }^{20}$ See Online Appendix G.
} 
The first highlighted inequality captures the strategic agency's incentive compatibility constraint; the second captures the firms' participation constraint. As $c<\frac{1}{2}$, a solution always exists: just choose $a=0$ to obtain $2 c \leq b \leq 1 .^{21}$ Moreover, as $\frac{\delta}{2-\delta}$ is increasing in $\delta$, raising $\delta$ relaxes the first constraint without affecting the second. The set of solutions therefore expands as $\delta$ increases. Note that upfront fees (corresponding to $a=b$ ) are socially optimal if $\delta>\frac{4 c}{1+2 c}$; contingent fees exclusively rewarding the agency at $r_{t}=1$ are socially optimal if $\delta>\frac{2+4 c}{3+2 c}$.

We next inquire: should we expect CRAs to choose a socially optimal fee structure (inducing $e_{t}=1$ each period), or might regulatory intervention be needed? To address this question, we extend our model by letting the agency make a take-it-or-leave-it offer $\left(\phi_{t}(1), \phi_{t}(-1)\right)$ to the firm in period $t$, specifying payments $\phi_{t}\left(r_{t}\right)$ as a function of $r_{t}{ }^{22}$ In line with the fact that rating agencies do not publish the fees that they charge their clients (see Kovbasyuk (2018) for a discussion of the pros and cons of transparent fees), we assume moreover that only firm $t$ can observe the agency's offer; ${ }^{23}$ we discuss later the importance of this feature.

The timing within period $t$ is here as follows (see Figure 4). First, the agency makes the offer $\left(\phi_{t}(1), \phi_{t}(-1)\right)$. If the firm rejects, the game moves on to the next period (we let $r_{t}=\emptyset$ denote the situation in which the agency's offer is rejected); if it accepts, the agency then decides whether or not to acquire information. Based on information gathered (if any), the agency publicly announces $r_{t}$, and receives $\phi_{t}\left(r_{t}\right)$. All players observe $q_{t}$ and the game moves on to the next period.

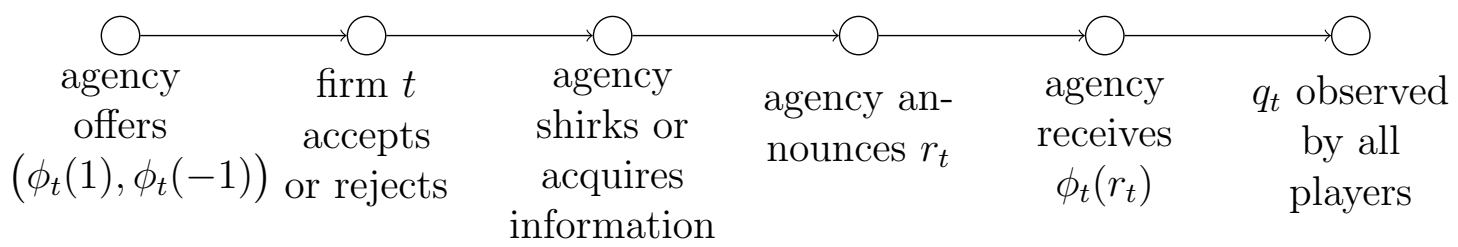

\section{Figure 4: Timeline With Endogenous FEeS}

\footnotetext{
${ }^{21}$ Intuitively, the rating $r_{t}=-1$ maximizes the likelihood that the agency acquired information. So rewarding the agency exclusively at $r_{t}=-1$ optimally incentivizes information acquisition.

${ }^{22}$ As noted by the European Securities and Markets Authority (ESMA) "ESMA is concerned that CRAs with significant market power could exploit their market power and margins, which gives them large space for fee flexibility. Costs are not currently the key pricing factor for these CRAs, which ultimately might result in price discrimination. [...] The fees charged to clients appear an estimation of the value for the individual client rather than being linked to the cost of production".

${ }^{23}$ The largest credit rating agencies provide broad guidelines concerning their rating fees (see, e.g. Standard \& Poor's Guidelines For Fees.), however fees actually paid by firms are not disclosed.
} 
A stationary strategy of the agency is a triple $((\phi(1, \cdot), \phi(-1, \cdot)), e(\cdot))$, where $(\phi(1, \cdot), \phi(-1, \cdot))$ : $[0,1] \rightarrow \mathbb{R}_{+}^{2}$ and $e:[0,1] \rightarrow[0,1]$ specifying, respectively, the agency's offer and the probability of acquiring information as functions of the agency's reputation $\rho_{t}$. A firms' stationary strategy is a mapping $d: \mathbb{R}^{2} \times[0,1] \rightarrow\{$ accept, reject $\}$ specifying which offers to accept as a function of the agency's reputation $\rho_{t}$.

We focus on equilibria such that, each period, the committed agency makes an offer which the firm accepts, but make no other assumptions concerning offers made by the committed type $^{24}$ The next definition adapts the equilibrium concept used previously; we refer to the model described here as the endogenous-fee setting.

Definition 2. An equilibrium with endogenous fees is a tuple $((\phi(1, \cdot), \phi(-1, \cdot)), e(\cdot), d(\cdot))$ such that, for all $\rho_{1}$ :

(i) in period $t$, the strategic agency makes the offer $\left(\phi\left(1, \rho_{t}\right), \phi\left(-1, \rho_{t}\right)\right)$;

(ii) the strategic agency acquires information with probability $e\left(\rho_{t}\right)$, and investors' beliefs satisfy

$$
\hat{e}_{t}=e\left(\rho_{t}\right)
$$

(iii) each firm's beliefs satisfy Bayes' rule whenever possible and $d\left(\left(\phi_{t}(1), \phi_{t}(-1)\right), \rho_{t}\right)=$ accept if and only if, upon observing $\left(\phi_{t}(1), \phi_{t}(-1)\right)$, firm $t$ expects a non-negative payoff from accepting the offer;

(iv) the strategy $((\phi(1, \cdot), \phi(-1, \cdot)), e(\cdot))$ maximizes the agency's expected intertemporal profit given the firms' decision rule and $\rho_{t+1}=\Psi\left(\rho_{t}, r_{t}, q_{t}\right)$, where

$$
\Psi\left(\rho_{t}, r_{t}, q_{t}\right):= \begin{cases}\rho_{t} & \text { if } q_{t}=1=r_{t} ; \\ \rho_{t}^{+} & \text {if } q_{t}=-1=r_{t} \text { and } \rho_{t}>0 ; \\ 0 & \text { if } q_{t}=-1=-r_{t}, \text { or } \rho_{t}=0, \text { or } r_{t}=\emptyset .\end{cases}
$$

(v) each period t the committed agency makes an offer that is accepted by the firm in period $t$.

\footnotetext{
${ }^{24}$ Exactly what offers the committed agency makes is irrelevant for this section's main result; the committed agency could make any offer $\left(\phi_{t}(1), \phi_{t}(-1)\right)$ satisfying

$$
\frac{1}{2} \mathbb{E}\left[q_{t} \mid r_{t}=1, \rho_{t}, e\left(\rho_{t}\right)\right] \geq \frac{\phi_{t}(1)+\phi_{t}(-1)}{2} .
$$
}


Note that with endogenous fees, firm $t$ 's beliefs at the time of deciding whether to accept or reject the offer $\left(\phi_{t}(1), \phi_{t}(-1)\right)$ could differ from investors' beliefs in period $t$. In equilibrium investors attach probability $\rho_{t}$ to the agency being committed and probability $e\left(\rho_{t}\right)$ to the strategic agency acquiring information. By contrast, the offer $\left(\phi_{t}(1), \phi_{t}(-1)\right)$ might affect firm t's beliefs concerning both the type of the agency, and the probability with which the strategic agency chooses to acquire information. ${ }^{25}$ The central remark is that, conditional on $r_{t}=1$, firm $t$ 's project is sold to investors at the price $\mathbb{E}\left[q_{t} \mid r_{t}=1, \rho_{t}, e\left(\rho_{t}\right)\right]$ irrespective of the agency's underlying offer to the firm. Hence, in equilibrium the offer $\left(\mathbb{E}\left[q_{t} \mid r_{t}=1, \rho_{t}, e\left(\rho_{t}\right)\right], 0\right)$ (i.e. the contingent fee of Section 2 with $\beta=1$ ) is acceptable irrespective of firm t's beliefs concerning the agency's type and of firm $t$ 's beliefs about the probability with which the strategic agency chooses to acquire information.

In fact, we show in the proof of the next theorem that the offer $\left(\mathbb{E}\left[q_{t} \mid r_{t}=1, \rho_{t}, e\left(\rho_{t}\right)\right], 0\right)$ is optimal for the agency in any equilibrium and that, in consequence, equilibrium offers induce the same amount of information acquisition as the contingent-fee structure of Section 2.

Theorem 2. Let $e^{*}(\cdot)$ denote the equilibrium with contingent fees and $\beta=1$ described in Proposition 1. In every equilibrium with endogenous fees, $e(\cdot)=e^{*}(\cdot)$. Moreover, an equilibrium with endogenous fees exists in which, for all $\rho_{t}$, the strategic agency makes the offer $\left(\mathbb{E}\left[q_{t} \mid r_{t}=1, \rho_{t}, e^{*}\left(\rho_{t}\right)\right], 0\right)$.

As information acquisition is here as in the contingent-fee setting of Section 2, social welfare is as in the equilibrium described in Proposition 1. Combining Proposition 1 and Theorem 2 thus establishes that the condition $\delta>\frac{2+4 c}{3+2 c}$ is both necessary and sufficient for the fee structure chosen by the strategic agency to be socially optimal. Why might the agency choose a sub-optimal fee structure? The key friction of this section's model is the non-observability (by investors) of the agency's offer $\left(\phi_{t}(1),\left(\phi_{t}(-1)\right)\right.$ to the firm in period $t$. If offers could be observed, then any socially optimal fee structure could be enforced by an equilibrium with endogenous fees. However, unobservable offers enable the strategic agency to (a) propose to the firm in period $t$ a contingent fee structure rewarding it at $r_{t}=1$, (b) shirk, and (c) obtain the maximal fee consistent with firm $t$ 's participation constraint. In this case, a contingent fee structure rewarding the agency at $r_{t}=1$ thus emerges even though it is socially inefficient.

\footnotetext{
${ }^{25}$ For instance if $\left(\phi_{t}(1), \phi_{t}(-1)\right)$ differs from the offer of the committed type then $\left(\phi_{t}(1), \phi_{t}(-1)\right)$ reveals that the agency is strategic.
} 


\section{Discussion}

In this section we discuss the policy implications of our results for the credit rating industry and the role of our main modeling assumptions.

Policy Implications. We remarked in Section 4 that moral hazard is the key friction making upfront fees potentially inefficient. A regulator can make information acquisition observable by directly monitoring CRAs' research activities. The resulting setting is analyzed in Online Appendix F. We show that in the absence of moral hazard the strategic agency always assigns correct ratings if fees are paid upfront, but not when fees are contingent. Upfront fees thus perhaps deserve the attention they received in the public debate: they are socially optimal provided the information acquisition process is supervised.

However, the analysis in Section 5 suggests alternative policy options. The regulator could leave information acquisition unsupervised, while making sure that the agency adopts a socially optimal fee structure. The regulator could directly impose a socially efficient fee structure, or else offer CRAs a way to publicly commit to whichever fee structure they prefer. Commitment could for example be achieved by supervising the transactions between agencies and rated firms.

All these policy options have advantages and shortcomings. Monitoring CRAs' research activities might be expensive, but offers the advantage that upfront fees ensure efficiency even if investors and the regulator are not fully aware of the agency's incentives. ${ }^{26}$ By contrast, the policy options that focus on the fee structure alone might be cheaper to implement, but require regulator and investors to have a correct notion of the CRA's incentives. ${ }^{27}$

Time Discounting. A discussion of time discounting is in order. To keep the model simple, we defined a period as the time elapsed between two ratings as well as the time it takes for the quality of a project to become public knowledge. This is a stark simplification, as in reality the two intervals have very different lengths. In markets for credit ratings the time between two ratings is extremely short, in the order of minutes. ${ }^{28}$ Public information about rated

\footnotetext{
${ }^{26}$ This policy ensures efficiency even if investors are uncertain about the value of $c$ and $\delta$. Furthermore, the regulator does not need to know the value of these parameters to implement upfront fees.

${ }^{27}$ As shown in Section 5, whether a fee structure is efficient or not depends on $c$ and $\delta$. On the one hand, regulating the fee structure while ignoring the value of these parameters might result in socially inefficient fee structures. On the other hand, letting the agency commit publicly to a fee structure, might result in the agency choosing a socially inefficient fee if investors are uncertain about $c$ and $\delta$.

${ }^{28}$ See evidence from White (2010), Table 1 . We thank an anonymous referee for raising this point.
} 
securities, on the contrary, takes far longer to reach the market. However, our results remain valid regardless of one's interpretation of the length of a period. If one interprets a period as a short time interval then our results are still valid as we show in Online Appendix E that they hold in a model in which firms and investors do not learn a project's quality before the next rating is assigned. Else one could interpret a period as a long time interval (say, a year) and interpret the revenues for a single rating as a proxy for the revenues from all the ratings assigned during that time interval.

If one interprets a period as a short time interval, then $\delta$ must be close to 1 . One might in this case worry about the relevance of the results discussed in Section 4 since, for $\delta$ sufficiently close to 1, Propositions 1 and 2 together show that the strategic agency acquires information with probability 1 regardless of the fee structure. Yet, this result hinges on the purely expositional choice to restrict attention to $c<\frac{\beta}{2}$ : we could extend the analysis to the case of $c \in\left(\frac{\beta}{2}, \frac{1}{2}\right)$, in which case upfront fees result in shirking for any $\delta<1$, while for $\delta$ sufficiently large (and $\rho$ not too large), contingent fees improve expected social welfare relative to upfront fees.

The choice to model a period both as the time elapsed between two ratings as well as the time it takes for the quality of a project to become public knowledge implies that in our model investors and firm learn about the agency's type by comparing ratings and project qualities. In reality, ratings assigned are likely to affect a CRA's reputation even when no information about the rated securities is learned. While our simplification implies that our model does not capture the full dynamics of a CRA's reputation, we show in Online Appendix E that our main results continue to hold even if $q_{t}$ is never revealed to firms and investors.

Rating-Based Regulation. In our model ratings have a purely informative role. In reality, credit ratings have a regulatory role, namely certain types of institutional investors can only buy assets with investment-level ratings, thus making a rating valuable regardless of its informational content. While adding rating-based regulation to our model is beyond the scope of this paper, we contend that our main result would still hold to the extent that the ratings have some informative content. Our analysis has no bite in markets where ratings are purchased purely in order to access a larger set of investors. 


\section{Conclusion}

This paper examined information acquisition by a credit rating agency with reputational concerns. Each period, the agency chooses whether or not to acquire costly information. The agency is either committed to acquire information, or behaves strategically with the objective of maximizing intertemporal profits. We first compare two compensation schemes. In one setting the agency receives a fee only if it assigns a favorable rating. This setting corresponds to the way rating agencies are currently compensated. In the other setting, the agency receives a fee irrespective of the rating assigned. Whereas fees paid upfront are socially optimal when information acquisition is publicly observable (or costless), moral hazard can lead contingent fees to induce higher social welfare than upfront fees. With endogenous fees, the contracts offered by the agency to the rated firms induce the agency to acquire information as if the fees were contingent. Our results contribute to the ongoing debate regarding the optimal way to compensate credit rating agencies. Specifically, our findings suggest that replacing contingent fees with upfront fees could be socially damaging in markets where all CRAs have become suspect, and in markets with many new CRAs having to build up their reputations.

The model could be extended in several directions. For example, the literature on competition among rating agencies shows how specific features of the market for credit ratings determine whether competition is feasible and desirable: along these lines, it would be interesting to evaluate the effect of the entry of new rating agencies under different compensation schemes. ${ }^{29}$ Alternatively, the analysis could be extended to study how different compensation schemes fare in markets in which each firm hires a rating agency multiple times. ${ }^{30}$

\footnotetext{
${ }^{29}$ Previous work on the effect of market structure on the quality of ratings includes Strausz (2005), FaureGrimaud, Peyrache and Quesada (2009), Bolton, Freixas and Shapiro (2012), Doherty, Kartasheva and Phillips (2012), Bouvard and Levy (2018), Hirth (2014), and Bizzotto (2014).

${ }^{30}$ As in Frenkel (2015), for example.
} 


\section{Appendix A}

Define $\Phi(\cdot, \cdot):[0,1] \times[0,1] \rightarrow \mathbb{R}$ by

$$
\Phi(\rho, e):=\frac{1-(1-\rho)(1-e)}{1+(1-\rho)(1-e)}
$$

Notice that $\mathbb{E}\left[q_{t} \mid r_{t}=1, \rho_{t}, \hat{e}_{t}\right]=\Phi\left(\rho_{t}, \hat{e}_{t}\right)$, and that $\Phi(\cdot, \cdot)$ is continuous, weakly increasing in both variables, $\Phi(\rho, e)>0$ unless $\rho=e=0$, and $\Phi(1, e)=\Phi(\rho, 1)=1$ for all $e$ and $\rho$ in $[0,1]$.

Given a function $e:[0,1] \rightarrow[0,1]$, define

$$
\rho^{+}:= \begin{cases}0 & \text { if } \rho=0 \\ \frac{\rho}{\rho+(1-\rho) e(\rho)} & \text { if } \rho>0 .\end{cases}
$$

Lemma 1. In any equilibrium, $e(0)=0$ and $V(0)=0$, where $V(\cdot)$ denotes the equilibrium value function. For all $\rho>0$,

$$
\begin{cases}\text { if } \quad \frac{\delta}{2} V\left(\rho^{+}\right)>\frac{\beta}{2} \Phi(\rho, e(\rho))+c \text { then } \quad e(\rho)=1 \\ \text { if } \quad \frac{\delta}{2} V\left(\rho^{+}\right)<\frac{\beta}{2} \Phi(\rho, e(\rho))+c \text { then } \quad e(\rho)=0\end{cases}
$$

Proof: By virtue of Bellman's Principle of Optimality, ${ }^{31}$

$$
V(\rho)=\max \left\{\beta \Phi(\rho, e(\rho))+\delta\left(\frac{1}{2} V(\rho)+\frac{1}{2} V(0)\right), \frac{\beta}{2} \Phi(\rho, e(\rho))-c+\delta\left(\frac{1}{2} V(\rho)+\frac{1}{2} V\left(\rho^{+}\right)\right)\right\},
$$

for all $\rho \in[0,1]$, and the agency's choice implied by $e(\rho)$ maximizes the right-hand side of the expression above. That is:

\footnotetext{
${ }^{31}$ Consider a state space $X$, a control space $U$, a law of motion $a$ such that $x_{t+1} \sim a\left(x_{t}, u_{t}\right)$, and a bounded reward function $r: X \times U \rightarrow \mathbb{R}$. Let $\pi: X \rightarrow U$ denote an arbitrary (stationary) policy, and define

$$
V(x):=\sup _{\pi} \mathbb{E}\left[\sum_{t=0}^{\infty} \delta^{t} r\left(x_{t}, u_{t}\right) \mid x_{0}=x\right] .
$$

Bellman's Principle of Optimality states that$$
V(x)=\sup _{u_{0}}\left[r\left(x_{0}, u_{0}\right)+\delta \mathbb{E}\left[V\left(x_{1}\right) \mid x_{0}=x, u_{0}\right]\right]
$$

and that $\pi$ is an optimal policy if and only if $\pi(x)$ maximizes the right-hand side of the expression above, for all $x \in X$.
} 


$$
\left\{\begin{array}{l}
e(\rho)=1 \quad \text { if } \quad \frac{\beta}{2} \Phi(\rho, e(\rho))-c+\delta\left(\frac{1}{2} V(\rho)+\frac{1}{2} V\left(\rho^{+}\right)\right)>\beta \Phi(\rho, e(\rho))+\delta\left(\frac{1}{2} V(\rho)+\frac{1}{2} V(0)\right) \\
e(\rho)=0 \quad \text { if } \quad \frac{\beta}{2} \Phi(\rho, e(\rho))-c+\delta\left(\frac{1}{2} V(\rho)+\frac{1}{2} V\left(\rho^{+}\right)\right)<\beta \Phi(\rho, e(\rho))+\delta\left(\frac{1}{2} V(\rho)+\frac{1}{2} V(0)\right) .
\end{array}\right.
$$

We are only left to show that $e(0)=0$ and $V(0)=0$. That $e(0)=0$ follows from the above, noting that $\rho^{+}=0$ if $\rho=0$. Then $V(0)=\beta \Phi(0,0)+\delta V(0)=\delta V(0)$. Hence $V(0)=0$.

Lemma 2. In any equilibrium,

$$
\left\{\begin{array}{l}
\text { if } e(\rho)=1 \text { then } V(\rho)=\frac{\frac{\beta}{2} \Phi(\rho, 1)-c}{1-\delta}=\max \left\{\frac{\frac{\beta}{2} \Phi(\rho, 1)-c}{1-\delta}, \frac{\beta \Phi(\rho, 1)}{1-\frac{\delta}{2}}\right\} \\
\text { if } e(\rho)<1 \text { then } V(\rho)=\frac{\beta \Phi(\rho, e(\rho))}{1-\frac{\delta}{2}}
\end{array}\right.
$$

Furthermore, $V(1)=\max \left\{\frac{\frac{\beta}{2} \Phi(1, e(1))-c}{1-\delta}, \frac{\beta \Phi(1, e(1))}{1-\frac{\delta}{2}}\right\} \geq V(\rho)$, for all $\rho \in[0,1]$.

Proof: The lemma follows from Bellman's Principle of Optimality together with the observations that (a) $e(\rho)=1$ implies $\rho^{+}=\rho$, (b) $\rho=1$ implies $\rho^{+}=1$, (c) $\Phi(1, e)=1$ for all $e \in[0,1]$, and $(\mathrm{d}) \Phi(\cdot, \cdot)$ is weakly increasing in both variables.

Proposition 3. If $\delta \geq \bar{\delta}:=\frac{2 \beta+4 c}{3 \beta+2 c}$ then

$$
e(\rho)=\left\{\begin{array}{l}
1 \text { if } \rho>0 \\
0 \text { if } \rho=0
\end{array}\right.
$$

is an equilibrium. If $\delta<\bar{\delta}$, in any equilibrium: $e(\rho)<1$ for all $\rho \in[0,1]$.

Proof: By Lemma 1, $e(0)=0$ for all $\delta$. Next, consider $\rho>0$. If in equilibrium $e(\rho)=1$ then by Lemma 1 and the observation that $\rho^{+}=\rho$ :

$$
\frac{\delta}{2} V(\rho) \geq \frac{\beta}{2} \Phi(\rho, 1)+c
$$

Applying Lemma 2 now yields

$$
\frac{\delta}{2}\left(\frac{\frac{\beta}{2} \Phi(\rho, 1)-c}{1-\delta}\right) \geq \frac{\beta}{2} \Phi(\rho, 1)+c
$$


or, equivalently, $\delta \geq \bar{\delta}$ once we note that $\Phi(\rho, 1)=1$. The condition $\delta \geq \bar{\delta}$ is thus necessary for $e(\rho)=1$. Sufficiency follows from the one-shot deviation principle.

Lemma 3. The following are equivalent:

$$
\begin{gathered}
\delta \geq \bar{\delta}, \\
\frac{\beta}{2} \Phi(\rho, 1)+c \leq \frac{\delta}{2}\left(\frac{\frac{\beta}{2} \Phi(\rho, 1)-c}{1-\delta}\right), \\
\frac{\beta}{2} \Phi(\rho, 1)+c \leq \frac{\delta}{2}\left(\frac{\beta \Phi(\rho, 1)}{1-\frac{\delta}{2}}\right), \\
\min \left\{\frac{\frac{\beta}{2} \Phi(\rho, 1)-c}{1-\delta}, \frac{\beta \Phi(\rho, 1)}{1-\frac{\delta}{2}}\right\}=\frac{\beta \Phi(\rho, 1)}{1-\frac{\delta}{2}} .
\end{gathered}
$$

Moreover, the equivalence between (10)-(12) continues to hold with strict inequalities instead of weak inequalities.

Proof: Equivalence is easily checked using $\Phi(\rho, 1)=1$.

Proposition 4. If $\delta>\bar{\delta}$ then (9) is the unique equilibrium.

Proof: By Lemma $1, e(0)=0$ in any equilibrium. So we are only left to show that, in any equilibrium, $e(\rho)=1$ for all $\rho>0$.

Suppose that an equilibrium exists such that $e(\hat{\rho})<1$ for some $\hat{\rho}>0$. Applying first Lemma 1 then Lemma 2:

$$
\frac{\beta}{2} \Phi(\hat{\rho}, e(\hat{\rho}))+c \geq \frac{\delta}{2} V\left(\hat{\rho}^{+}\right) \geq \frac{\delta}{2}\left(\frac{\beta \Phi\left(\hat{\rho}^{+}, e\left(\hat{\rho}^{+}\right)\right)}{1-\frac{\delta}{2}}\right) .
$$

We thus obtain, using the equivalence between (10) and (12) (with strict inequalities), the following sequence of inequalities:

$$
\frac{\delta}{2}\left(\frac{\beta \Phi(\hat{\rho}, 1)}{1-\frac{\delta}{2}}\right)>\frac{\beta}{2} \Phi(\hat{\rho}, 1)+c \geq \frac{\beta}{2} \Phi(\hat{\rho}, e(\hat{\rho}))+c \geq \frac{\delta}{2}\left(\frac{\beta \Phi\left(\hat{\rho}^{+}, e\left(\hat{\rho}^{+}\right)\right)}{1-\frac{\delta}{2}}\right),
$$

from which we infer that $e\left(\hat{\rho}^{+}\right)<1$. We can thus repeat the steps above with $\hat{\rho}^{+}$instead of $\hat{\rho}$, and so on. This process determines a sequence $\left\{\rho_{n}\right\}$ such that, for all $n$ : 
(i) $e\left(\rho_{n}\right)<1$,

(ii) $\rho_{n+1}=\frac{\rho_{n}}{\rho_{n}+\left(1-\rho_{n}\right) e\left(\rho_{n}\right)}>\rho_{n}$,

(iii) $\frac{\beta}{2} \Phi\left(\rho_{n}, e\left(\rho_{n}\right)\right)+c \geq \frac{\delta}{2}\left(\frac{\beta \Phi\left(\rho_{n+1}, e\left(\rho_{n+1}\right)\right)}{1-\frac{\delta}{2}}\right)$.

By (i)-(ii), either $e\left(\rho_{n}\right) \rightarrow 1$ or $\rho_{n} \rightarrow 1$. Hence, taking limits in (iii) yields (using continuity of $\Phi(\cdot, \cdot)$ and the fact that $\Phi(1, e)=\Phi(\rho, 1)=1$ for all $e$ and $\rho$ in $[0,1])$ :

$$
\frac{\beta}{2} \Phi(1,1)+c \geq \frac{\delta}{2}\left(\frac{\beta \Phi(1,1)}{1-\frac{\delta}{2}}\right) .
$$

The equivalence between (10) and (12) (with strict inequalities) establishes a contradiction between (14) and $\delta>\bar{\delta}$.

Proposition 5. If $\delta \leq \underline{\delta}:=\frac{2 c}{\beta+c}$ then $e(\rho)=0$ for all $\rho \in[0,1]$ is the unique equilibrium.

Proof: Note first that $\delta \leq \underline{\delta}$ if and only if

$$
c \geq \frac{\delta}{2}\left(\frac{\beta \Phi(1, e(1))}{1-\frac{\delta}{2}}\right) .
$$

Next, the assumption $\beta>2 c>0$ implies $\underline{\delta}<\bar{\delta}$; combining Lemmas 2 and 3 thus shows that, in any equilibrium,

$$
V(1)=\frac{\beta \Phi(1, e(1))}{1-\frac{\delta}{2}}
$$

whenever $\delta<\underline{\delta}$. Combining (15), (16) and Lemma 2 now yields $c>\frac{\delta}{2} V(\rho)$, for all $\rho \in[0,1)$. Hence, by Lemma $1, e(\rho)=0$, for all $\rho \in[0,1]$.

That $e(\rho)=0$ for all $\rho \in[0,1]$ is an equilibrium is immediate from (15), (16), and the one-shot deviation principle.

Proposition 6. Let $\delta \in(\underline{\delta}, \bar{\delta})$. There exists a unique equilibrium. In equilibrium,

$$
\begin{cases}e(\rho)=0 & \text { if } \rho \in\{0\} \cup[\tilde{\rho}, 1], \\ e(\rho) \in(0, \tilde{e}] & \text { if } \rho \in(0, \tilde{\rho}),\end{cases}
$$


where $\tilde{\rho} \in(0,1)$ and $\tilde{e} \in(0,1)$ are defined implicitly by

$$
\frac{\beta}{2} \Phi(\tilde{\rho}, 0)+c=\frac{\delta}{2}\left(\frac{\beta \Phi(1,0)}{1-\frac{\delta}{2}}\right)
$$

and

$$
\frac{\beta}{2} \Phi(0, \tilde{e})+c=\frac{\delta}{2}\left(\frac{\beta \Phi(0,1)}{1-\frac{\delta}{2}}\right)
$$

respectively.

Proof: Applying Lemma 3,

$$
\delta<\bar{\delta} \Leftrightarrow \frac{\beta}{2} \Phi(\rho, 1)+c>\frac{\delta}{2}\left(\frac{\beta \Phi(\rho, 1)}{1-\frac{\delta}{2}}\right) .
$$

Moreover, by (15),

$$
\delta>\underline{\delta} \Leftrightarrow c<\frac{\delta}{2}\left(\frac{\beta \Phi(\rho, 1)}{1-\frac{\delta}{2}}\right) .
$$

Thus $\tilde{\rho} \in(0,1)$ and $\tilde{e} \in(0,1)$.

We now prove the rest of the proposition. We will first proceed by induction to show that there can exist at most one equilibrium. We will then argue that the inductive procedure yields an equilibrium. As a preliminary step, observe that by Lemma 2 and the equivalence between (10) and (13), an equilibrium must satisfy:

$$
V(\rho)=\frac{\beta \Phi(\rho, e(\rho))}{1-\frac{\delta}{2}}
$$

for all $\rho \in[0,1]$.

The inductive procedure starts as follows. Combining (18) and (20), any equilibrium must be such that, for all $\rho>\tilde{\rho}$ :

$$
\frac{\beta}{2} \Phi(\rho, 0)+c>\frac{\delta}{2} V\left(\rho^{+}\right) .
$$

Thus, by Lemma 1, if an equilibrium exists it must satisfy $e(\rho)=0$ for all $\rho>\tilde{\rho}$. A similar argument shows that in fact the same must be true for $\rho=\tilde{\rho}$.

By contrast, consider $\rho \in(0, \tilde{\rho})$. The combination of (18), (20), and Lemma 1 shows that $e(\rho)=0$ is impossible in equilibrium. Similarly, the combination of (19), (20) and Lemma 1 shows that $e(\rho)>\tilde{e}$ is impossible in equilibrium. Thus, any equilibrium must satisfy (17). By 
virtue of Lemma 1 this in turn implies that the indifference condition

$$
\frac{\delta}{2} V\left(\rho^{+}\right)=\frac{\beta}{2} \Phi(\rho, e(\rho))+c
$$

must hold for all $\rho \in(0, \tilde{\rho})$.

Next define $\rho_{1}<\tilde{\rho}$ such that

$$
\tilde{\rho}=\frac{\rho_{1}}{\rho_{1}+\left(1-\rho_{1}\right) \tilde{e}} .
$$

By construction of $\rho_{1}$ and property (17), in any equilibrium: $\rho^{+} \geq \tilde{\rho}$ for all $\rho \in\left[\rho_{1}, \tilde{\rho}\right)$. (21), (17) and (20) now pin down a unique candidate equilibrium $e(\rho)$ for each $\rho \in\left[\rho_{1}, \tilde{\rho}\right)$. Moreover, this candidate equilibrium is continuous in $\rho$ and such that $\Phi(\rho, e(\rho))$ is increasing in $\rho$. Repeating the step above with $\rho_{1}$ instead of $\tilde{\rho}$ yields $\rho_{2}<\rho_{1}$ and a unique candidate equilibrium $e(\rho)$ for each $\rho \in\left[\rho_{2}, \rho_{1}\right)$, and so on. This defines a sequence $\left\{\rho_{n}\right\}$ where, for all $n, \tilde{\rho}_{n}=\frac{\rho_{n+1}}{\rho_{n+1}+\left(1-\rho_{n+1}\right) \tilde{e}}$. As $\tilde{e}<1, \rho_{n} \rightarrow 0$. This inductive procedure therefore pins down a unique candidate equilibrium. That this candidate equilibrium is in fact an equilibrium is a consequence of the one-shot deviation principle.

Proof of Proposition 1: Follows from Propositions 3-6. 


\section{Appendix B}

Define $\Upsilon(\cdot, \cdot):[0,1] \times[0,1] \rightarrow \mathbb{R}$ by

$$
\Upsilon(\rho, e):=\frac{1}{2}(1-(1-\rho)(1-e)) .
$$

Notice that $\mathbb{P}\left(r_{t}=1 \mid \rho_{t}, \hat{e}_{t}\right) \mathbb{E}\left[q_{t} \mid r_{t}=1, \rho_{t}, \hat{e}_{t}\right]=\Upsilon\left(\rho_{t}, \hat{e}_{t}\right)$, and that $\Upsilon(\cdot, \cdot)$ is continuous, weakly increasing in both variables, $\Upsilon(\rho, e)>0$ unless $\rho=e=0$, and $\Upsilon(1, e)=\Upsilon(\rho, 1)=\frac{1}{2}$ for all $e$ and $\rho$ in $[0,1]$. We also borrow the definition of $\rho^{+}$from Appendix A.

Lemma 4. In any equilibrium, $e(0)=0$ and $V(0)=0$, where $V(\cdot)$ denotes the equilibrium value function. For all $\rho>0$,

$$
\left\{\begin{array}{l}
\text { if } \quad \frac{\delta}{2} V\left(\rho^{+}\right)>c \text { then } \quad e(\rho)=1 \\
\text { if } \quad \frac{\delta}{2} V\left(\rho^{+}\right)<c \text { then } \quad e(\rho)=0
\end{array}\right.
$$

Proof: By virtue of Bellman's Principle of Optimality,

$$
V(\rho)=\max \left\{\beta \Upsilon(\rho, e(\rho))+\delta\left(\frac{1}{2} V(\rho)+\frac{1}{2} V(0)\right), \beta \Upsilon(\rho, e(\rho))-c+\delta\left(\frac{1}{2} V(\rho)+\frac{1}{2} V\left(\rho^{+}\right)\right)\right\},
$$

for all $\rho \in[0,1]$, and the choice implied by $e(\rho)$ maximizes the right-hand side of $(22)$. That is:

$$
\left\{\begin{array}{l}
e(\rho)=1 \quad \text { if } \quad \beta \Upsilon(\rho, e(\rho))-c+\delta\left(\frac{1}{2} V(\rho)+\frac{1}{2} V\left(\rho^{+}\right)\right)>\beta \Upsilon(\rho, e(\rho))+\delta\left(\frac{1}{2} V(\rho)+\frac{1}{2} V(0)\right), \\
e(\rho)=0 \quad \text { if } \quad \beta \Upsilon(\rho, e(\rho))-c+\delta\left(\frac{1}{2} V(\rho)+\frac{1}{2} V\left(\rho^{+}\right)\right)<\beta \Upsilon(\rho, e(\rho))+\delta\left(\frac{1}{2} V(\rho)+\frac{1}{2} V(0)\right) .
\end{array}\right.
$$

We are only left to show that $e(0)=0$ and $V(0)=0$. That $e(0)=0$ follows from the above, noting that $\rho^{+}=0$ if $\rho=0$. Substituting back into (22) then yields $V(0)=\beta \Phi(0,0)+\delta V(0)=$ $\delta V(0)$. Hence $V(0)=0$.

Lemma 5. In any equilibrium,

$$
\left\{\begin{array}{l}
\text { if } e(\rho)=1 \text { then } V(\rho)=\frac{\beta \Upsilon(\rho, 1)-c}{1-\delta}=\max \left\{\frac{\beta \Upsilon(\rho, 1)-c}{1-\delta}, \frac{\beta \Upsilon(\rho, 1)}{1-\frac{\delta}{2}}\right\}, \\
\text { if } e(\rho)<1 \text { then } V(\rho)=\frac{\beta \Upsilon(\rho, e(\rho))}{1-\frac{\delta}{2}}
\end{array}\right.
$$


Furthermore, $V(1)=\max \left\{\frac{\beta \Upsilon(1, e(1))-c}{1-\delta}, \frac{\beta \Upsilon(1, e(1))}{1-\frac{\delta}{2}}\right\} \geq V(\rho)$, for all $\rho \in[0,1]$.

Proof: The lemma follows from Bellman's Principle of Optimality together with the observations that $(\mathrm{a}) e(\rho)=1$ implies $\rho^{+}=\rho$, (b) $\rho=1$ implies $\rho^{+}=1$, (c) $\Upsilon(1, e)=\frac{1}{2}$ for all $e \in[0,1]$, and $(\mathrm{d}) \Upsilon(\cdot, \cdot)$ is weakly increasing in both variables.

Proposition 7. If $\delta>\frac{4 c}{\beta+2 c}$, then

$$
e(\rho)=\left\{\begin{array}{l}
1 \text { if } \rho>0 \\
0 \text { if } \rho=0
\end{array}\right.
$$

is an equilibrium. If $\delta<\frac{4 c}{\beta+2 c}$, in any equilibrium: $e(\rho)<1$ for all $\rho \in[0,1]$.

Proof: By Lemma 4, $e(0)=0$ for all $\delta$. Next, consider $\rho>0$. If in equilibrium $e(\rho)=1$ then Lemma 4 and the observation that $\rho^{+}=\rho$ yield

$$
\frac{\delta}{2} V(\rho) \geq c
$$

Applying Lemma 5 now gives

$$
\frac{\delta}{2}\left(\frac{\beta \Upsilon(\rho, 1)-c}{1-\delta}\right) \geq c,
$$

or, equivalently, $\delta \geq \frac{4 c}{\beta+2 c}$ once we note that $\Upsilon(\rho, 1)=\frac{1}{2}$. The condition $\delta \geq \frac{4 c}{\beta+2 c}$ is thus necessary for $e(\rho)=1$. Sufficiency follows from the one-shot deviation principle.

Lemma 6. The following are equivalent:

$$
\begin{gathered}
\delta \geq \frac{4 c}{\beta+2 c}, \\
c \leq \frac{\delta}{2}\left(\frac{\beta \Upsilon(\rho, 1)-c}{1-\delta}\right), \\
c \leq \frac{\delta}{2}\left(\frac{\beta \Upsilon(\rho, 1)}{1-\frac{\delta}{2}}\right), \\
\min \left\{\frac{\beta \Upsilon(\rho, 1)-c}{1-\delta}, \frac{\beta \Upsilon(\rho, 1)}{1-\frac{\delta}{2}}\right\}=\frac{\beta \Upsilon(\rho, 1)}{1-\frac{\delta}{2}} .
\end{gathered}
$$


Moreover, the equivalence between (24)-(26) continues to hold with strict inequalities instead of weak inequalities.

Proof: Equivalence is easily checked using $\Upsilon(\rho, 1)=\frac{1}{2}$.

Proposition 8. If $\delta>\frac{4 c}{\beta+2 c}$, then (23) is the unique equilibrium.

Proof: By Lemma $4, e(0)=0$ in any equilibrium. So we are only left to show that, in any equilibrium, $e(\rho)=1$ for all $\rho>0$.

Suppose an equilibrium exists such that $e(\hat{\rho})<1$ for some $\hat{\rho}>0$. Applying first Lemma 4 then Lemma 5:

$$
c \geq \frac{\delta}{2} V\left(\hat{\rho}^{+}\right) \geq \frac{\delta}{2}\left(\frac{\beta \Upsilon\left(\hat{\rho}^{+}, e\left(\hat{\rho}^{+}\right)\right)}{1-\frac{\delta}{2}}\right) .
$$

We thus obtain, using equivalence of (24) and (26) (with strict inequalities), the following sequence of inequalities:

$$
\frac{\delta}{2}\left(\frac{\beta \Upsilon(\hat{\rho}, 1)}{1-\frac{\delta}{2}}\right)>c \geq \frac{\delta}{2}\left(\frac{\beta \Upsilon\left(\hat{\rho}^{+}, e\left(\hat{\rho}^{+}\right)\right)}{1-\frac{\delta}{2}}\right)
$$

from which we infer that $e\left(\hat{\rho}^{+}\right)<1$. We can thus repeat the steps above with $\hat{\rho}^{+}$instead of $\hat{\rho}$, and so on. This process determines a sequence $\left\{\rho_{n}\right\}$ such that, for all $n$ :

(i) $e\left(\rho_{n}\right)<1$,

(ii) $\rho_{n+1}=\frac{\rho_{n}}{\rho_{n}+\left(1-\rho_{n}\right) e\left(\rho_{n}\right)}>\rho_{n}$,

(iii) $c \geq \frac{\delta}{2}\left(\frac{\beta \Upsilon\left(\rho_{n+1}, e\left(\rho_{n+1}\right)\right)}{1-\frac{\delta}{2}}\right)$.

By (i)-(ii), either $e\left(\rho_{n}\right) \rightarrow 1$ or $\rho_{n} \rightarrow 1$. Hence, taking limits in (iii) yields (using continuity of $\Upsilon(\cdot, \cdot)$ and the fact that $\Upsilon(1, e)=\Upsilon(\rho, 1)=\frac{1}{2}$ for all $e$ and $\rho$ in $\left.[0,1]\right)$ :

$$
c \geq \frac{\delta}{2}\left(\frac{\beta \Upsilon(1,1)}{1-\frac{\delta}{2}}\right) .
$$

The equivalence between (24) and (26) (with strict inequalities) establishes a contradiction with (28). 
Proposition 9. If $\delta<\frac{4 c}{\beta+2 c}$ then $e(\rho)=0$ for all $\rho \in[0,1]$ is the unique equilibrium.

Proof: By Lemma 6, we have $\delta<\frac{4 c}{\beta+2 c}$ if and only if

$$
c>\frac{\delta}{2}\left(\frac{\beta \Upsilon(1, e(1))}{1-\frac{\delta}{2}}\right) .
$$

Next, combining Lemmas 5 and 6 yields

$$
V(1)=\frac{\beta \Upsilon(1, e(1))}{1-\frac{\delta}{2}} .
$$

Combining (29) and (30) gives $c>\frac{\delta}{2} V(1)$; hence, by Lemma $5, c>\frac{\delta}{2} V(\rho)$ for all $\rho \in[0,1]$. Lemma 4 thus yields $e(\rho)=0$, for all $\rho \in[0,1]$.

That $e(\rho)=0$, for all $\rho \in[0,1]$ is an equilibrium is immediate from (29), (30), and the one-shot deviation principle.

Proof of Proposition 2: Follows from Propositions 7-9.

Proof of Theorem 1: Proposition 1 characterizes the unique equilibrium with contingent fees, and Proposition 2 characterizes the unique equilibrium with upfront fees. The cutoff $\bar{\delta}=\frac{2 \beta+4 c}{3 \beta+2 c}$ is taken from Proposition 3. The cutoff $\underline{\delta}=\frac{2 c}{\beta+c}$ is taken from Proposition 5 . With upfront fees, either the strategic agency shirks irrespective of $\rho_{t}$ or the strategic agency acquires information with probability 1 irrespective of $\rho_{t}$. Hence, all that remains to show is that the expected period- $t$ welfare is an increasing function of the probability with which the strategic agency chooses to acquire information.

The expected period- $t$ welfare is

$$
\begin{aligned}
\mathbb{P}\left(r_{t}=1\right) \mathbb{E}\left[q_{t} \mid r_{t}=1\right]-c\left(\rho_{t}+\left(1-\rho_{t}\right) e\left(\rho_{t}\right)\right)= & \left(\frac{1}{2} \cdot 1+\frac{1}{2}\left(1-\rho_{t}\right)\left(1-e\left(\rho_{t}\right)\right)\right) \frac{1-\left(1-\rho_{t}\right)\left(1-e\left(\rho_{t}\right)\right)}{1+\left(1-\rho_{t}\right)\left(1-e\left(\rho_{t}\right)\right)} \\
& -c\left(\rho_{t}+\left(1-\rho_{t}\right) e\left(\rho_{t}\right)\right) \\
= & \left(\frac{1}{2}-c\right)\left(\rho_{t}+\left(1-\rho_{t}\right) e\left(\rho_{t}\right)\right)
\end{aligned}
$$

the result follows, since $c<\frac{1}{2}$. 


\section{Appendix $\mathrm{C}$}

Proof of Theorem 2: We start with the first part of the theorem, namely, we show that $e(\cdot)=e^{*}(\cdot)$ in any equilibrium. Consider an arbitrary equilibrium $((\phi(1, \cdot), \phi(-1, \cdot)), e(\cdot), d(\cdot))$, denoted by $E$. We separate the analysis into three cases.

Case 1: in $E$, for all $\rho_{t}$, the offer of the committed type differs from $\left(\phi\left(1, \rho_{t}\right), \phi\left(-1, \rho_{t}\right)\right)$. In $E$, the offer $\left(\mathbb{E}\left[q_{t} \mid r_{t}=1, \rho_{t}, e\left(\rho_{t}\right)\right], 0\right)$ is acceptable since no matter firm $t$ 's beliefs the firm can recoup with investors the fee paid to the agency. Therefore,

$$
\left(1-\frac{e\left(\rho_{t}\right)}{2}\right) \phi\left(1, \rho_{t}\right)+\frac{e\left(\rho_{t}\right)}{2} \phi\left(-1, \rho_{t}\right) \geq\left(1-\frac{e\left(\rho_{t}\right)}{2}\right) \mathbb{E}\left[q_{t} \mid r_{t}=1, \rho_{t}, e\left(\rho_{t}\right)\right]
$$

otherwise offering $\left(\mathbb{E}\left[q_{t} \mid r_{t}=1, \rho_{t}, e\left(\rho_{t}\right)\right], 0\right)$ and acquiring information with probability $e\left(\rho_{t}\right)$ would be a strictly profitable deviation for the agency. On the other hand, in $E$, the offer $\left(\phi\left(1, \rho_{t}\right), \phi\left(-1, \rho_{t}\right)\right)$ is acceptable as well. Therefore,

$$
\left(1-\frac{e\left(\rho_{t}\right)}{2}\right) \mathbb{E}\left[q_{t} \mid r_{t}=1, \rho_{t}, e\left(\rho_{t}\right)\right] \geq\left(1-\frac{e\left(\rho_{t}\right)}{2}\right) \phi\left(1, \rho_{t}\right)+\frac{e\left(\rho_{t}\right)}{2} \phi\left(-1, \rho_{t}\right),
$$

since $\left(1-\frac{e\left(\rho_{t}\right)}{2}\right) \mathbb{E}\left[q_{t} \mid r_{t}=1, \rho_{t}, e\left(\rho_{t}\right)\right]$ is firm $t$ 's expected revenue from investors following the offer $\left(\phi\left(1, \rho_{t}\right), \phi\left(-1, \rho_{t}\right)\right)$ (the offer of the committed type differs from $\left(\phi\left(1, \rho_{t}\right), \phi\left(-1, \rho_{t}\right)\right)$, so firm $t$ assigns probability 1 to the strategic type upon observing $\left.\left(\phi\left(1, \rho_{t}\right), \phi\left(-1, \rho_{t}\right)\right)\right)$. Combining the previous inequalities,

$$
\left(1-\frac{e\left(\rho_{t}\right)}{2}\right) \mathbb{E}\left[q_{t} \mid r_{t}=1, \rho_{t}, e\left(\rho_{t}\right)\right]=\left(1-\frac{e\left(\rho_{t}\right)}{2}\right) \phi\left(1, \rho_{t}\right)+\frac{e\left(\rho_{t}\right)}{2} \phi\left(-1, \rho_{t}\right) .
$$

We now claim that $E^{\prime} \equiv\left(\left(\mathbb{E}\left[q_{t} \mid r_{t}=1, \rho_{t}, e\left(\rho_{t}\right)\right], 0\right), e(\cdot), d(\cdot)\right)$ comprises an equilibrium, with off-path beliefs as in equilibrium $E$ wherever possible, or such that firm $t$ assigns probability 1 to the agency being strategic and acquiring information with probability $e\left(\rho_{t}\right)$ in case $\left(\left(\mathbb{E}\left[q_{t} \mid r_{t}=1, \rho_{t}, e\left(\rho_{t}\right)\right], 0\right) \neq\left(\phi\left(1, \rho_{t}\right), \phi\left(-1, \rho_{t}\right)\right)\right.$ and the agency makes the offer $\left(\phi\left(1, \rho_{t}\right), \phi\left(-1, \rho_{t}\right)\right)$. Note first that, by $(31), E$ and $E^{\prime}$ induce identical expected payoffs on their respective equilibrium paths. Thus $E$ and $E^{\prime}$ also induce identical continuation payoffs. That $E^{\prime}$ comprises an equilibrium now follows from the one-shot deviation principle. However, if $E^{\prime}$ is an equilibrium then $e(\cdot)=e^{*}(\cdot)$ by virtue of Proposition 1. 
Case 2: in $E$, for all $\rho_{t}$, both types of the agency offer $\left(\phi\left(1, \rho_{t}\right), \phi\left(-1, \rho_{t}\right)\right)$. In $E$, the offer $\left(\mathbb{E}\left[q_{t} \mid r_{t}=1, \rho_{t}, e\left(\rho_{t}\right)\right], 0\right)$ is acceptable. So $\phi\left(-1, \rho_{t}\right)=0$ trivially implies $\phi\left(1, \rho_{t}\right)=\mathbb{E}\left[q_{t} \mid r_{t}=\right.$ $\left.1, \rho_{t}, e\left(\rho_{t}\right)\right]$. In what follows, we consider the case $\phi\left(-1, \rho_{t}\right)>0$. First,

$$
\left(1-\frac{e\left(\rho_{t}\right)}{2}\right) \phi\left(1, \rho_{t}\right)+\frac{e\left(\rho_{t}\right)}{2} \phi\left(-1, \rho_{t}\right) \geq\left(1-\frac{e\left(\rho_{t}\right)}{2}\right) \mathbb{E}\left[q_{t} \mid r_{t}=1, \rho_{t}, e\left(\rho_{t}\right)\right],
$$

otherwise offering $\left(\mathbb{E}\left[q_{t} \mid r_{t}=1, \rho_{t}, e\left(\rho_{t}\right)\right], 0\right)$ and acquiring information with probability $e\left(\rho_{t}\right)$ would be a strictly profitable deviation for the agency. On the other hand, in $E$, the offer $\left(\phi\left(1, \rho_{t}\right), \phi\left(-1, \rho_{t}\right)\right)$ is acceptable as well. Therefore,

$$
\begin{aligned}
& {\left[\frac{\rho_{t}}{2}+\left(1-\rho_{t}\right)\left(1-\frac{e\left(\rho_{t}\right)}{2}\right)\right] \mathbb{E}\left[q_{t} \mid r_{t}=1, \rho_{t}, e\left(\rho_{t}\right)\right]} \\
& \quad \geq \rho_{t}\left(\frac{\phi\left(1, \rho_{t}\right)+\phi\left(-1, \rho_{t}\right)}{2}\right)+\left(1-\rho_{t}\right)\left[\left(1-\frac{e\left(\rho_{t}\right)}{2}\right) \phi\left(1, \rho_{t}\right)+\frac{e\left(\rho_{t}\right)}{2} \phi\left(-1, \rho_{t}\right)\right],
\end{aligned}
$$

or, equivalently,

$$
\begin{aligned}
\frac{\rho_{t}}{2}\left[\mathbb { E } \left[q_{t} \mid r_{t}\right.\right. & \left.\left.=1, \rho_{t}, e\left(\rho_{t}\right)\right]-\phi\left(1, \rho_{t}\right)-\phi\left(-1, \rho_{t}\right)\right] \\
& \geq\left(1-\rho_{t}\right)\left[\left(1-\frac{e\left(\rho_{t}\right)}{2}\right) \phi\left(1, \rho_{t}\right)+\frac{e\left(\rho_{t}\right)}{2} \phi\left(-1, \rho_{t}\right)-\left(1-\frac{e\left(\rho_{t}\right)}{2}\right) \mathbb{E}\left[q_{t} \mid r_{t}=1, \rho_{t}, e\left(\rho_{t}\right)\right]\right] .
\end{aligned}
$$

By (32), the right-hand side in the previous inequality is non-negative, giving $\mathbb{E}\left[q_{t} \mid r_{t}=\right.$ $\left.1, \rho_{t}, e\left(\rho_{t}\right)\right] \geq \phi\left(1, \rho_{t}\right)+\phi\left(-1, \rho_{t}\right)$. Thus,

$$
\left(1-\frac{e\left(\rho_{t}\right)}{2}\right) \mathbb{E}\left[q_{t} \mid r_{t}=1, \rho_{t}, e\left(\rho_{t}\right)\right] \geq\left(1-\frac{e\left(\rho_{t}\right)}{2}\right) \phi\left(1, \rho_{t}\right)+\left(1-\frac{e\left(\rho_{t}\right)}{2}\right) \phi\left(-1, \rho_{t}\right) .
$$

The latter inequality yields $e\left(\rho_{t}\right)=1$ since, given $\phi\left(-1, \rho_{t}\right)>0, e\left(\rho_{t}\right)<1$ would imply

$$
\left(1-\frac{e\left(\rho_{t}\right)}{2}\right) \mathbb{E}\left[q_{t} \mid r_{t}=1, \rho_{t}, e\left(\rho_{t}\right)\right]>\left(1-\frac{e\left(\rho_{t}\right)}{2}\right) \phi\left(1, \rho_{t}\right)+\frac{e\left(\rho_{t}\right)}{2} \phi\left(-1, \rho_{t}\right)
$$

and contradict (32). We therefore obtain

$$
\left(1-\frac{e\left(\rho_{t}\right)}{2}\right) \mathbb{E}\left[q_{t} \mid r_{t}=1, \rho_{t}, e\left(\rho_{t}\right)\right] \geq\left(1-\frac{e\left(\rho_{t}\right)}{2}\right) \phi\left(1, \rho_{t}\right)+\frac{e\left(\rho_{t}\right)}{2} \phi\left(-1, \rho_{t}\right),
$$


which, combined with (32), yields

$$
\left(1-\frac{e\left(\rho_{t}\right)}{2}\right) \phi\left(1, \rho_{t}\right)+\frac{e\left(\rho_{t}\right)}{2} \phi\left(-1, \rho_{t}\right)=\left(1-\frac{e\left(\rho_{t}\right)}{2}\right) \mathbb{E}\left[q_{t} \mid r_{t}=1, \rho_{t}, e\left(\rho_{t}\right)\right]
$$

We now claim that $E^{\prime} \equiv\left(\left(\mathbb{E}\left[q_{t} \mid r_{t}=1, \rho_{t}, e\left(\rho_{t}\right)\right], 0\right), e(\cdot), d(\cdot)\right)$ comprises an equilibrium, with off-path beliefs as in equilibrium $E$. First, by (33), $E$ and $E^{\prime}$ induce identical expected payoffs on their respective equilibrium paths. Thus $E$ and $E^{\prime}$ also induce identical continuation payoffs. That $E^{\prime}$ comprises an equilibrium now follows from the one-shot deviation principle, combined with the observation that in $E^{\prime}$ the offer $\left(\phi\left(1, \rho_{t}\right), \phi\left(-1, \rho_{t}\right)\right)$ of the committed type is still acceptable (since we showed that $e\left(\rho_{t}\right)=1$ ). However, if $E^{\prime}$ is an equilibrium then $e(\cdot)=e^{*}(\cdot)$ by virtue of Proposition 1 .

Case 3: general case. The case in which for some values of $\rho_{t}$ both types of the agency offer $\left(\phi\left(1, \rho_{t}\right), \phi\left(-1, \rho_{t}\right)\right)$, while the offer of the committed type differs from $\left(\phi\left(1, \rho_{t}\right), \phi\left(-1, \rho_{t}\right)\right)$ for other values of $\rho_{t}$ is a straightforward extension of Cases 1 and 2 above.

We next show the second part of the theorem, that is, we show that an equilibrium with endogenous fees exists in which the strategic agency makes offers $\left(\mathbb{E}\left[q_{t} \mid r_{t}=1, \rho_{t}, e^{*}\left(\rho_{t}\right)\right], 0\right)$. Let $V_{c}(\cdot)$ denote the value function of the agency in the unique equilibrium of Proposition 1 with $\beta=1$. To fix ideas, assume that each period the committed type makes the offer $\left(\mathbb{E}\left[q_{t} \mid r_{t}=1, \rho_{t}, e^{*}\left(\rho_{t}\right)\right], 0\right)$ (other cases are similar). Moreover, suppose that investors' beliefs satisfy $\hat{e}_{t}=e^{*}\left(\rho_{t}\right)$ (we are not claiming at this stage that these beliefs are consistent). For each offer $\omega \neq\left(\mathbb{E}\left[q_{t} \mid r_{t}=1, \rho_{t}, e^{*}\left(\rho_{t}\right)\right], 0\right)$ accepted in period $t$ let $\tilde{e}\left(\omega, \rho_{t}\right)$ denote an optimal information acquisition strategy of the agency given the continuation payoffs $V_{c}(\cdot)$ (accepting $\omega$ need not be optimal for firm $t)$. Let $d\left(\left(\mathbb{E}\left[q_{t} \mid r_{t}=1, \rho_{t}, e^{*}\left(\rho_{t}\right)\right], 0\right), \rho_{t}\right)=$ accept and, for all $\omega \neq\left(\mathbb{E}\left[q_{t} \mid r_{t}=1, \rho_{t}, e^{*}\left(\rho_{t}\right)\right], 0\right), d\left(\omega, \rho_{t}\right)=$ accept if and only if firm $t$ 's expected payoff from accepting $\omega$ is non-negative given beliefs that the agency is strategic and acquires information with probability $\tilde{e}\left(\omega, \rho_{t}\right)$.

We claim that $\left(\left(\mathbb{E}\left[q_{t} \mid r_{t}=1, \rho_{t}, e^{*}\left(\rho_{t}\right)\right], 0\right), e^{*}\left(\rho_{t}\right), d\left(\rho_{t}\right)\right)$ constitutes an equilibrium, with off-path beliefs such that upon observing an offer $\omega \neq\left(\mathbb{E}\left[q_{t} \mid r_{t}=1, \rho_{t}, e^{*}\left(\rho_{t}\right)\right], 0\right)$ firm $t$ assigns probability 1 to the agency being strategic and acquiring information with probability $\tilde{e}\left(\omega, \rho_{t}\right)$. By virtue of the one-shot deviation principle all that we need to show is that no one-shot deviation gives the agency strictly greater expected payoff than following the strat- 
egy $\left(\left(\mathbb{E}\left[q_{t} \mid r_{t}=1, \rho_{t}, e^{*}\left(\rho_{t}\right)\right], 0\right), e^{*}\left(\rho_{t}\right)\right)$. One-shot deviations to an offer which firm $t$ rejects are clearly unprofitable. So we examine a one-shot deviation to an offer $\omega=\left(\phi_{\omega}(1), \phi_{\omega}(-1)\right)$ which firm $t$ accepts. The most profitable such deviation entails acquiring information with probability $\tilde{e}\left(\omega, \rho_{t}\right)$. The corresponding expected payoff is

$$
\tilde{e}\left(\omega, \rho_{t}\right)\left[\frac{1}{2}\left(\phi_{\omega}(1)+\phi_{\omega}(-1)\right)-c+\frac{\delta}{2}\left(V_{c}\left(\rho_{t}\right)+V_{c}\left(\rho_{t}^{+}\right)\right)\right]+\left(1-\tilde{e}\left(\omega, \rho_{t}\right)\right)\left[\phi_{\omega}(1)+\frac{\delta}{2} V_{c}\left(\rho_{t}\right)\right] .
$$

On the other hand, the offer $\omega$ being acceptable (under the belief that the agency is strategic and acquires information with probability $\left.\tilde{e}\left(\omega, \rho_{t}\right)\right)$,

$$
\left(1-\frac{\tilde{e}\left(\omega, \rho_{t}\right)}{2}\right) \mathbb{E}\left[q_{t} \mid r_{t}=1, \rho_{t}, e^{*}\left(\rho_{t}\right)\right] \geq\left(1-\frac{\tilde{e}\left(\omega, \rho_{t}\right)}{2}\right) \phi_{\omega}(1)+\frac{\tilde{e}\left(\omega, \rho_{t}\right)}{2} \phi_{\omega}(-1) .
$$

The latter inequality implies that the strategic agency can obtain at least the expected payoff (34) by making the offer $\left(\mathbb{E}\left[q_{t} \mid r_{t}=1, \rho_{t}, e^{*}\left(\rho_{t}\right)\right], 0\right)$ and acquiring information with probability $\tilde{e}\left(\omega, \rho_{t}\right)$. As with offer $\left(\mathbb{E}\left[q_{t} \mid r_{t}=1, \rho_{t}, e^{*}\left(\rho_{t}\right)\right], 0\right)$ the information acquisition probability $e^{*}\left(\rho_{t}\right)$ is optimal, combining the previous remarks shows that no one-shot deviation to an offer $\omega$ which firm $t$ accepts is strictly profitable for the agency. 


\section{References}

Benabou, R. and Laroque, G. (1992) Using privileged information to manipulate markets: Insiders, gurus, and credibility, The Quarterly Journal of Economics, 107, 921-958.

Bizzotto, J. (2014) Certifier Competition and Product Quality, Mimeo.

Board, S. and Meyer-ter Vehn, M. (2013) Reputation for quality, Econometrica, 81, 23812462.

Bolton, P., Freixas, X. and Shapiro, J. (2012) The credit ratings game, The Journal of Finance, LXVII, 85-111.

Bongaerts, D. (2014) Alternatives for issuer-paid credit rating agencies.

Bouvard, M. and Levy, R. (2018) Two-sided reputation in certification markets, Management Science.

Chade, H. and Kovrijnykh, N. (2016) Delegated information acquisition with moral hazard, Journal of Economic Theory, 162, 55-92.

Doherty, N. a., Kartasheva, A. V. and Phillips, R. D. (2012) Information effect of entry into credit ratings market: The case of insurers' ratings, Journal of Financial Economics, 106, 308-330.

Faure-Grimaud, A., Peyrache, E. and Quesada, L. (2009) The ownership of ratings, The RAND Journal of Economics, 40, 234-257.

Frenkel, S. (2015) Repeated interaction and rating inflation: A model of double reputation, American Economic Journal: Microeconomics, 7, 250-280.

Fulghieri, P., Strobl, G. and Xia, H. (2014) The economics of solicited and unsolicited credit ratings, The Review of Financial Studies, 27, 484-518.

Goldstein, I. and Huang, C. (2017) Credit rating inflation and firms' investments, Mimeo.

Hirth, S. (2014) Credit rating dynamics and competition, Journal of Banking \& Finance, 49, $100-112$. 
Inderst, R. and Ottaviani, M. (2012) Financial Advice, Journal of Economic Literature, 50, 494-512.

Jeon, D.-S. and Lovo, S. (2013) Credit rating industry: A helicopter tour of stylized facts and recent theories, International Journal of Industrial Organization, 31, 643-651.

Kashyap, A. K. and Kovrijnykh, N. (2016) Who Should Pay for Credit Ratings and How?, Review of Financial Studies, 29, 420-456.

Kovbasyuk, S. (2018) Seller-Paid Ratings, Mimeo.

Kreps, D. M. and Wilson, R. (1982) Reputation and Imperfect Information, Journal of Economic Theory, 279, 253-279.

Mathis, J., McAndrews, J. and Rochet, J.-C. (2009) Rating the raters: Are reputation concerns powerful enough to discipline rating agencies?, Journal of Monetary Economics, 56, 657-674.

Milgrom, P. and Roberts, J. (1982) Predation, reputation, and entry deterrence, Journal of Economic Theory, 312, 280-312.

Opp, C. C., Opp, M. M. and Harris, M. (2013) Rating agencies in the face of regulation, Journal of Financial Economics, 108, 46-61.

Sangiorgi, F. and Spatt, C. (2016) Opacity, credit rating shopping, and bias, Management Science, 63, 4016-4036.

Sangiorgi, F. and Spatt, C. (2017) The economics of credit rating agencies, Foundations and Trends® in Finance, 12, 1-116.

Skreta, V. and Veldkamp, L. (2009) Ratings shopping and asset complexity: A theory of ratings inflation, Journal of Monetary Economics, 56, 678-695.

Strausz, R. (2005) Honest certification and the threat of capture, International Journal of Industrial Organization, 23, 45-62.

United States Securities and Exchange Commission (2012) Report to Congress on Assigned Credit Ratings. 
United States Senate (2011) Wall Street and the Financial Crisis: Anatomy of a Financial Collapse.

White, L. (2010) Markets: The credit rating agencies, Journal of Economic Perspectives, 24, $211-226$. 


\section{Online Appendix A}

In this appendix we check the robustness of our analysis by relaxing the assumption on the perfect observability of project returns in case the agency chooses to become informed. We show that, as in the baseline model:

- for some parameter values contingent fees improve expected social welfare relative to upfront fees;

- upfront fees improve expected social welfare relative to contingent fees as long as the cost of information acquisition, $c$, is sufficiently small.

The Model. The agency lives for two periods, $t=1,2 .{ }^{32}$ For concreteness, we distinguish now between a project's quality, $q_{t} \in\{-1,1\}$, and a project's return, $Q_{t}$. By acquiring information in period $t$, the agency observes a noisy signal $s_{t} \in\{-1,1\}$ of the project quality $q_{t}$, such that $\mathbb{P}\left(s_{t}=q_{t} \mid q_{t}\right)=1-\epsilon$, with $\epsilon \in\left(0, \frac{1}{2}\right)$. A project's return is related to its quality by $Q_{t}=\frac{q_{t}}{1-2 \epsilon} \cdot{ }^{33}$ As in the baseline model, the sequence $\left\{q_{t}\right\}$ is independent and identically distributed according to $\mathbb{P}\left(q_{t}=1\right)=\frac{1}{2}$. The baseline model therefore corresponds to $\epsilon=0$.

Applying Bayes' rule gives

$$
\mathbb{E}\left[q_{t} \mid r_{t}=1, \rho_{t}, \hat{e}_{t}\right]=\Phi\left(\rho_{t}, \hat{e}_{t}\right):=\frac{1-\left(1-\rho_{t}\right)\left(1-\hat{e}_{t}\right)}{1+\left(1-\rho_{t}\right)\left(1-\hat{e}_{t}\right)}
$$

and

$$
\mathbb{P}\left(r_{t}=1 \mid \rho_{t}, \hat{e}_{t}\right) \mathbb{E}\left[q_{t} \mid r_{t}=1, \rho_{t}, \hat{e}_{t}\right]=\Upsilon\left(\rho_{t}, \hat{e}_{t}\right):=\frac{1}{2}\left[1-\left(1-\rho_{t}\right)\left(1-\hat{e}_{t}\right)\right]
$$

Next, given $e:[0,1] \rightarrow[0,1]$, define

$$
\begin{aligned}
& \rho^{+}:=\left\{\begin{array}{ll}
0 & \text { if } \rho=0 \\
\frac{\rho}{\rho+(1-\rho) e(\rho)} & \text { if } \rho>0
\end{array} ;\right. \\
& \rho^{b}:= \begin{cases}0 & \text { if } \rho=0 \\
\frac{\rho \epsilon}{\epsilon+(1-\epsilon)(1-\rho)(1-e(\rho))} & \text { if } \rho>0\end{cases}
\end{aligned}
$$

The next definition is immediately adapted from the equilibrium concept of Section 2.

\footnotetext{
${ }^{32}$ We set here $\beta=1$ to reduce notation.

${ }^{33}$ We scale up project returns by a factor equal to $\frac{1}{1-2 \epsilon}$ to keep the expected return conditional on observing $s_{t}$ precisely equal to $s_{t}$.
} 
Definition 3. An equilibrium with contingent fees comprises functions $e_{t}:[0,1] \rightarrow[0,1]$ specifying the probabilities $e_{t}\left(\rho_{t}\right)$ that the strategic agency acquires information in period $t$ given reputation $\rho_{t}$, for $t=1,2$, such that each period:

(i) the choice(s) implied by $e_{t}\left(\rho_{t}\right)$ maximize the agency's expected intertemporal profit given

$$
\pi_{t}=\Phi\left(\rho_{t}, \hat{e}_{t}\right) \mathbf{1}_{\left\{r_{t}=1\right\}}-c \mathbf{1}_{\{\text {information acquired in period } t\}}
$$

and

$$
\rho_{2}= \begin{cases}\rho_{1}^{g} & \text { if } q_{1}=1=r_{1} ; \\ \rho_{1}^{+} & \text {if } q_{1}=-1=r_{1} ; \\ \rho_{1}^{b} & \text { if } q_{1}=-1=-r_{1} .\end{cases}
$$

(ii) firms and investors' beliefs satisfy $\hat{e}_{t}=e_{t}\left(\rho_{t}\right)$.

The definition of an equilibrium with upfront fees is obtained by replacing (35) with

$$
\pi_{t}=\Upsilon\left(\rho_{t}, \hat{e}_{t}\right)-c \mathbf{1}_{\{\text {information acquired in period } t\}}
$$

The Equilibria. We next characterize the equilibria with, respectively, contingent fees and upfront fees. With 2 periods the strategic agency always shirks in period 2. So the focus is on information acquisition at $t=1$. Proofs of the propositions are relegated to the end of this appendix.

Proposition 10. With contingent fees, in equilibrium $e_{1}\left(\rho_{1}\right)>0$ if and only if $\delta>2 c$ and $\rho_{1}<\rho_{c}(\delta)$, where $\rho_{c}(\delta)$ is defined implicitly by

$$
\delta=\left(c+\frac{\rho_{c}}{2\left(2-\rho_{c}\right)}\right)\left(\frac{2-(1+\epsilon) \rho_{c}}{2-(1+2 \epsilon(1-\epsilon)) \rho_{c}}\right)\left(\frac{2-(2-\epsilon) \rho_{c}}{1-\rho_{c}}\right) .
$$

Moreover $\lim _{c \rightarrow 0} e_{1}\left(\rho_{1}\right)<1$ for any $\rho_{1}$ and $\delta$.

Proposition 11. With upfront fees, in equilibrium $e_{1}\left(\rho_{1}\right)>0$ if and only if $\delta>4 c$ and $\rho_{1}<\rho_{u}(\delta)$, where $\rho_{u}(\delta)$ is defined implicitly by

$$
\delta=4 c\left(\frac{1}{1-2 \epsilon(1-\epsilon) \rho_{u}}+\frac{\epsilon(1-\epsilon) \rho_{u}^{2}}{\left(1-\rho_{u}\right)\left(1-2 \epsilon(1-\epsilon) \rho_{u}\right)}\right) .
$$


Moreover $\lim _{c \rightarrow 0} e_{1}\left(\rho_{1}\right)=1$ for any $\rho_{1}$ and $\delta$.

We illustrate the propositions in Figure 5 . At $t=1$ the strategic agency acquires information, with some probability, for all $\left(\delta, \rho_{1}\right)$ lying to the right of the curve $\rho_{c}$ when fees are contingent and to the right of the curve $\rho_{u}$ when fees are upfront.

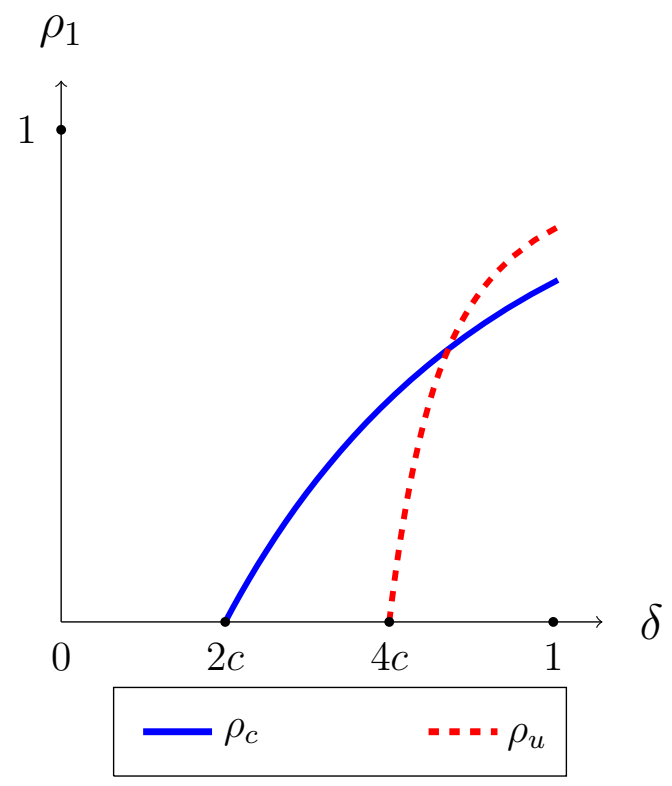

Figure 5: Noisy Signals of PROJECT RETURNS

Welfare Comparison. As in equilibrium the strategic agency shirks at $t=2$ irrespective of the fee structure, comparing expected social welfare in equilibrium under different fee structures reduces to comparing the probability that a strategic agency acquires information at $t=1$. When fees are contingent, for $\delta \in(2 c, 4 c)$ the strategic agency acquires information at $t=1$ with positive probability provided its reputation is not too high (Proposition 10). By contrast, for $\delta \in(2 c, 4 c)$ the strategic agency shirks with probability 1 when fees are upfront (Proposition 11). For $\delta \in(2 c, 4 c)$ and sufficiently low reputation expected social welfare is therefore higher under contingent fees than under upfront fees. Moreover for any $\delta$ and $\rho_{1}$, $\lim _{c \rightarrow 0} e_{1}\left(\rho_{1}\right)=1$ only in the case of upfront fees. Thus upfront fees increase expected social welfare if the cost of information acquisition is sufficiently low. 
Proof of Proposition 10: First, note that the function $\Phi$ is continuous, weakly increasing in each of its arguments, and $\Phi(1, \cdot)=\Phi(\cdot, 1)=1$. Moreover, notice that in any equilibrium the strategic agency must shirk at $t=2$ with probability 1 giving $\pi_{2}=\Phi\left(\rho_{2}, 0\right)=\frac{\rho_{2}}{2-\rho_{2}}$. Define $V(\rho):=\frac{\rho}{2-\rho}$. At $t=1$, the agency's expected intertemporal profit from shirking and announcing $r_{t}=1$ is equal to $\Phi\left(\rho_{1}, \hat{e}_{1}\right)+\frac{\delta}{2}\left[V\left(\rho_{1}^{g}\right)+V\left(\rho_{1}^{b}\right)\right]$. The intertemporal profit from acquiring information is equal to $\frac{1}{2} \Phi\left(\rho_{1}, \hat{e}_{1}\right)-c+\frac{\delta}{2}\left[V\left(\rho_{1}^{+}\right)+(1-\epsilon) V\left(\rho_{1}^{g}\right)+\epsilon V\left(\rho_{1}^{b}\right)\right]$.

Fix $\rho_{1} \in(0,1)$. An equilibrium in which $e_{1}\left(\rho_{1}\right)=1$ exists if and only if

$$
\frac{1}{2} \Phi\left(\rho_{1}, 1\right)+\frac{\delta}{2}\left[\epsilon V\left(\rho_{1}\right)+(1-\epsilon) V\left(\rho_{1}\right)\right] \leq \frac{\delta}{2} V\left(\rho_{1}\right)-c .
$$

This condition is always violated, thus in equilibrium $e_{1}\left(\rho_{1}\right)<1$. An equilibrium in which $e_{1}\left(\rho_{1}\right)=0$ in turn exists if and only if

$$
\frac{1}{2} \Phi\left(\rho_{1}, 0\right)+\frac{\delta}{2}\left[\epsilon V\left(\frac{\rho_{1}(1-\epsilon)}{1-\epsilon \rho_{1}}\right)+(1-\epsilon) V\left(\frac{\rho_{1} \epsilon}{1-(1-\epsilon) \rho_{1}}\right)\right] \geq \frac{\delta}{2} V(1)-c .
$$

Substituting for $\Phi(\cdot)$ and $V(\cdot)$ and simplifying yields

$$
\delta \leq \tilde{\delta}\left(\rho_{1}\right):=\left(c+\frac{\rho_{1}}{2\left(2-\rho_{1}\right)}\right)\left(\frac{2-(1+\epsilon) \rho_{1}}{2-(1+2 \epsilon(1-\epsilon)) \rho_{1}}\right)\left(\frac{2-(2-\epsilon) \rho_{1}}{1-\rho_{1}}\right) .
$$

Notice that $\tilde{\delta}\left(\rho_{1}\right)$ is continuous and increasing in $\rho_{1}$ for $\rho_{1} \in(0,1)$ as it is the product of 3 terms, each of which is continuous and increasing in $\rho_{1}$ for $\rho_{1} \in(0,1)$. Hence $\delta<\tilde{\delta}\left(\rho_{1}\right)$ is equivalent to $\rho_{1}>\rho_{c}(\delta)$; moreover as $\tilde{\delta}(0)=2 c$, then $e_{1}\left(\rho_{1}\right)>0$ only if $\delta>2 c$. Furthermore, an equilibrium in which $e_{1}\left(\rho_{1}\right) \in(0,1)$ exists if and only if

$$
\begin{aligned}
\frac{\delta}{2}\left[V\left(\frac{\rho_{1}}{\rho_{1}+\left(1-\rho_{1}\right) e\left(\rho_{1}\right)}\right)\right. & -\epsilon V\left(\frac{\rho_{1}(1-\epsilon)}{1-\epsilon+\epsilon\left(1-\rho_{1}\right)\left(1-e\left(\rho_{1}\right)\right)}\right) \\
& \left.-(1-\epsilon) V\left(\frac{\rho_{1} \epsilon}{\epsilon+(1-\epsilon)\left(1-\rho_{1}\right)\left(1-e\left(\rho_{1}\right)\right)}\right)\right]=c+\frac{1}{2} \Phi\left(\rho_{1}, e\left(\rho_{1}\right)\right) .
\end{aligned}
$$

The left-hand side of (37) is strictly decreasing in $e\left(\rho_{1}\right)$, while the right-hand side is strictly increasing in $e\left(\rho_{1}\right)$, thus there is at most one $e\left(\rho_{1}\right)$ that satisfies the equality. Moreover, for $e\left(\rho_{1}\right)=0$ this equality reduces to $\delta=\tilde{\delta}\left(\rho_{1}\right)$. As the left-hand side of (37) is increasing in $\delta$ and the right-hand side does not depend on $\delta$, then $\delta>\tilde{\delta}\left(\rho_{1}\right)$, which is equivalent to $\rho_{1}<\rho_{c}(\delta)$, is necessary for an equilibrium in which $e_{1}\left(\rho_{1}\right) \in(0,1)$. That this condition is also sufficient follows from standard arguments. 
Pick a pair $\delta$ and $\rho_{1}$. For sufficiently small $c, \delta>\tilde{\delta}\left(\rho_{1}\right)$, thus in equilibrium $e_{1}\left(\rho_{1}\right)$ satisfies (37). Note also that for $c=0$ the unique $e\left(\rho_{1}\right)$ that satisfies (37) belongs to the interval $(0,1)$. As both $\Phi(\cdot)$ and $V(\cdot)$ are continuous in each of their arguments, then in equilibrium $\lim _{c \rightarrow 0} e_{1}\left(\rho_{1}\right)<1$.

Proof of Proposition 11: First notice that $\Upsilon$ is continuous, weakly increasing in each of its arguments, and $\Upsilon(1, \cdot)=\Upsilon(\cdot, 1)=\frac{1}{2}$. Moreover, note that in any equilibrium the strategic agency must shirk at $t=2$ with probability 1 , giving $\pi_{2}=\Upsilon\left(\rho_{2}, 0\right)=\frac{\rho_{2}}{2}$. Define $V(\rho):=\frac{\rho}{2}$. At $t=1$, the agency's expected intertemporal profit from shirking and announcing $r_{t}=1$ is equal to $\Upsilon\left(\rho_{1}, \hat{e}_{1}\right)+\frac{\delta}{2}\left[V\left(\rho_{1}^{g}\right)+V\left(\rho_{1}^{b}\right)\right]$. The intertemporal profit from acquiring information is equal to $\Upsilon\left(\rho_{1}, \hat{e}_{1}\right)-c+\frac{\delta}{2}\left[V\left(\rho_{1}^{+}\right)+(1-\epsilon) V\left(\rho_{1}^{g}\right)+\epsilon V\left(\rho_{1}^{b}\right)\right]$. Fix $\rho_{1} \in(0,1)$. An equilibrium in which $e_{1}\left(\rho_{1}\right)=1$ exists if and only if

$$
\frac{\delta}{2}\left[\epsilon V\left(\rho_{1}\right)+(1-\epsilon) V\left(\rho_{1}\right)\right] \leq \frac{\delta}{2} V\left(\rho_{1}\right)-c
$$

This condition is always violated. An equilibrium in which $e_{1}\left(\rho_{1}\right)=0$ exists if and only if

$$
\frac{\delta}{2}\left[\epsilon V\left(\frac{\rho_{1}(1-\epsilon)}{1-\epsilon \rho_{1}}\right)+(1-\epsilon) V\left(\frac{\rho_{1} \epsilon}{1-(1-\epsilon) \rho_{1}}\right)\right] \geq \frac{\delta}{2} V(1)-c
$$

Substituting for $V(\cdot)$ and simplifying yields

$$
\delta \leq \bar{\delta}\left(\rho_{1}\right):=4 c\left(\frac{1}{1-2 \epsilon(1-\epsilon) \rho_{1}}+\frac{\epsilon(1-\epsilon) \rho_{1}^{2}}{\left(1-\rho_{1}\right)\left(1-2 \epsilon(1-\epsilon) \rho_{1}\right)}\right)
$$

As $\bar{\delta}\left(\rho_{1}\right)$ is continuous and increasing in $\rho_{1}$, then $\delta<\bar{\delta}\left(\rho_{1}\right)$ is equivalent to $\rho_{1}>\rho_{u}(\delta)$; moreover, as $\bar{\delta}(0)=4 c$, then $e_{1}\left(\rho_{1}\right)>0$ only if $\delta>4 c$. Furthermore, an equilibrium in which $e_{1}\left(\rho_{1}\right) \in(0,1)$ exists if and only if

$$
\begin{aligned}
\frac{\delta}{2}\left[V\left(\frac{\rho_{1}}{\rho_{1}+\left(1-\rho_{1}\right) e\left(\rho_{1}\right)}\right)\right. & -\epsilon V\left(\frac{\rho_{1}(1-\epsilon)}{1-\epsilon+\epsilon\left(1-\rho_{1}\right)\left(1-e\left(\rho_{1}\right)\right)}\right) \\
& \left.-(1-\epsilon) V\left(\frac{\rho_{1} \epsilon}{\epsilon+(1-\epsilon)\left(1-\rho_{1}\right)\left(1-e\left(\rho_{1}\right)\right)}\right)\right]=c
\end{aligned}
$$

The left-hand side of (38) is strictly decreasing in $e\left(\rho_{1}\right)$, and the right-hand side is strictly 
increasing in $e\left(\rho_{1}\right)$, thus there is at most one $e\left(\rho_{1}\right)$ that satisfies the equality. Moreover, for $e\left(\rho_{1}\right)=0$ this equality reduces to $\delta=\bar{\delta}\left(\rho_{1}\right)$. As the left-hand side is increasing in $\delta$ and the left-hand side does not depend on $\delta, \delta>\bar{\delta}\left(\rho_{1}\right)$ is necessary for an equilibrium in which $e_{1}\left(\rho_{1}\right) \in(0,1)$. That this condition is also sufficient follows from standard arguments.

Next, fix $\delta$ and $\rho_{1}$. For sufficiently small $c, \delta>\bar{\delta}\left(\rho_{1}\right)$, thus in equilibrium $e_{1}\left(\rho_{1}\right)$ satisfies (38). Note also that, for $c=0, e\left(\rho_{1}\right)=1$ satisfies (38). Continuity of $\Phi$ thus ensures that in equilibrium $\lim _{c \rightarrow 0} e_{1}\left(\rho_{1}\right)=1$. 


\section{Online Appendix B}

In this appendix we check the robustness of our analysis by allowing the agency to announce $r_{t}=-1$ whether or not in period $t$ the agency chooses to acquire information. We show that, as in the baseline model:

- for some parameter values contingent fees improve expected social welfare relative to upfront fees;

- upfront fees improve expected social welfare relative to contingent fees as long as the cost of information acquisition, $c$, is sufficiently small.

The Model. The agency lives for two periods, $t=1,2 .{ }^{34}$ We let $\hat{z}_{t}$ denote the beginning-ofperiod- $t$ belief that the strategic agency will announce $r_{t}=-1$ in case it shirks. ${ }^{35}$ Applying Bayes' rule,

$$
\mathbb{E}\left[q_{t} \mid r_{t}=1, \rho_{t}, \hat{e}_{t}, \hat{z}_{t}\right]=\Phi\left(\rho_{t}, \hat{e}_{t}, \hat{z}_{t}\right):=\frac{1-\left(1-\rho_{t}\right)\left(1-\hat{e}_{t}\right)}{1+\left(1-\rho_{t}\right)\left(1-\hat{e}_{t}\right)\left(1-2 \hat{z}_{t}\right)}
$$

while

$$
\mathbb{E}\left[q_{t} \mid r_{t}=-1, \rho_{t}, \hat{e}_{t}, \hat{z}_{t}\right]=\frac{(-1)\left(1-\left(1-\rho_{t}\right)\left(1-\hat{e}_{t}\right)\right)}{\rho_{t}+\left(1-\rho_{t}\right) \hat{e}_{t}+2 \hat{z}_{t}\left(1-\rho_{t}\right)\left(1-\hat{e}_{t}\right)} .
$$

Note that $\mathbb{E}\left[q_{t} \mid r_{t}=1, \rho_{t}, \hat{e}_{t}, \hat{z}_{t}\right] \geq 0 \geq \mathbb{E}\left[q_{t} \mid r_{t}=-1, \rho_{t}, \hat{e}_{t}, \hat{z}_{t}\right]$ for all $\rho_{t}, \hat{e}_{t}$ and $\hat{z}_{t}$. So with contingent fees the period- $t$ profit of the agency is

$$
\pi_{t}=\Phi\left(\rho_{t}, \hat{e}_{t}, \hat{z}_{t}\right) \mathbf{1}_{\left\{r_{t}=1\right\}}-c \mathbf{1}_{\{\text {information acquired in period } t\}}
$$

Applying Bayes' rule again,

$$
\mathbb{P}\left(r_{t}=1 \mid \rho_{t}, \hat{e}_{t}, \hat{z}_{t}\right) \mathbb{E}\left[q_{t} \mid r_{t}=1, \rho_{t}, \hat{e}_{t}, \hat{z}_{t}\right]=\Upsilon\left(\rho_{t}, \hat{e}_{t}\right):=\frac{1}{2}\left[1-\left(1-\rho_{t}\right)\left(1-\hat{e}_{t}\right)\right]
$$

With upfront fees, the period- $t$ profit of the agency is

$$
\pi_{t}=\Upsilon\left(\rho_{t}, \hat{e}_{t}\right)-c \mathbf{1}_{\{\text {information acquired in period } t\}} .
$$

\footnotetext{
${ }^{34}$ We set here $\beta=1$ to reduce notation.

${ }^{35}$ As noted in the baseline model, upon acquiring information the strategic agency would report truthfully even if it had the option to misreport. Thus we assume here, as in the baseline model, that upon acquiring information the agency reports truthfully.
} 
Given functions $e_{t}(\cdot)$ and $z_{t}(\cdot)$ from $[0,1]$ to $[0,1]$, define

$$
\rho_{t}^{+}:= \begin{cases}0 & \text { if } \rho_{t}=0 \\ \frac{\rho_{t}}{\rho_{t}+\left(1-\rho_{t}\right)\left[e_{t}\left(\rho_{t}\right)+\left(1-e_{t}\left(\rho_{t}\right)\right)\left(1-z_{t}\left(\rho_{t}\right)\right)\right]} & \text { if } \rho_{t}>0\end{cases}
$$

and

$$
\rho_{t}^{++}:= \begin{cases}0 & \text { if } \rho_{t}=0 \\ \frac{\rho_{t}}{\rho_{t}+\left(1-\rho_{t}\right)\left[e\left(\rho_{t}\right)+\left(1-e\left(\rho_{t}\right)\right) z_{t}\left(\rho_{t}\right)\right]} & \text { if } \rho_{t}>0 .\end{cases}
$$

The next definition is immediately adapted from the equilibrium concept of Section 2.

Definition 4. An equilibrium with contingent fees comprises functions $e_{t}:[0,1] \rightarrow[0,1]$ and $z_{t}:[0,1] \rightarrow[0,1]$, for $t=1,2$, specifying the probabilities $e_{t}\left(\rho_{t}\right)$ that the strategic agency acquires information and $z_{t}\left(\rho_{t}\right)$ of announcing $r_{t}=-1$ conditional on shirking, given reputation $\rho_{t}$, such that each period:

(i) the choices implied by $e_{t}\left(\rho_{t}\right)$ and $z_{t}\left(\rho_{t}\right)$ maximize the agency's expected intertemporal profit given (39) and

$$
\rho_{2}= \begin{cases}\rho_{1}^{+} & \text {if } q_{1}=r_{1}=1 \\ \rho_{1}^{++} & \text {if } q_{1}=r_{1}=-1 \\ 0 & \text { if } q_{1}=-r_{1} .\end{cases}
$$

(ii) firms and investors' beliefs satisfy $\hat{e}_{t}=e_{t}\left(\rho_{t}\right)$ and $\hat{z}_{t}=z_{t}\left(\rho_{t}\right)$.

The definition of an equilibrium with upfront fees is obtained replacing (39) with (40).

The Equilibria. We next characterize the equilibria with, respectively, contingent fees and upfront fees. With 2 periods the strategic agency always shirks in period 2. So the focus is on information acquisition at $t=1$. Proofs of the propositions are relegated to the end of this appendix.

Proposition 12. Let fees be contingent. In equilibrium $e_{1}\left(\rho_{1}\right)<1$ for all $\rho_{1} \in(0,1)$. Moreover, $e_{1}\left(\rho_{1}\right) \in(0,1)$ for $\delta>\frac{2 c-1+\sqrt{1+12 c+4 c^{2}}}{2}$ and $\rho_{1} \in\left(\frac{2 c(2+\delta)}{1+2 \delta+\delta^{2}}, \frac{4 c-2 \delta}{2 c-1-\delta}\right)$. 
Proposition 13. Let fees be upfront. In equilibrium, if $\delta \leq 4 c$ then $e_{1}\left(\rho_{1}\right)=0$ for all $\rho_{1} \in(0,1)$. If instead $\delta>4 c$ then

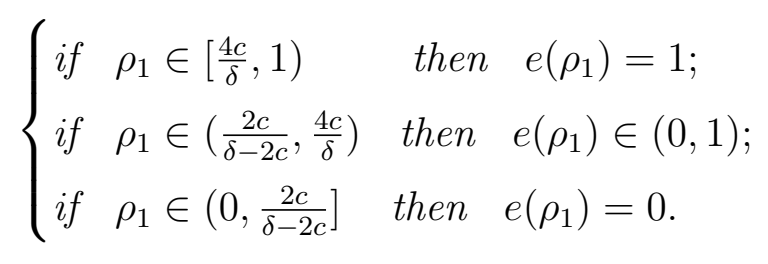

We illustrate in Figure 6 the regions in $\left(\delta, \rho_{1}\right)$-space identified in the two propositions. When fees are contingent, at $t=1$ the strategic agency acquires information, with some probability, for all $\left(\delta, \rho_{1}\right)$ lying to the right of the solid curves. When fees are upfront, the strategic agency acquires information, with some probability, for all $\left(\delta, \rho_{1}\right)$ in between the dashed lines, and acquires information with probability 1 for all $\left(\delta, \rho_{1}\right)$ to the right of both dashed lines.

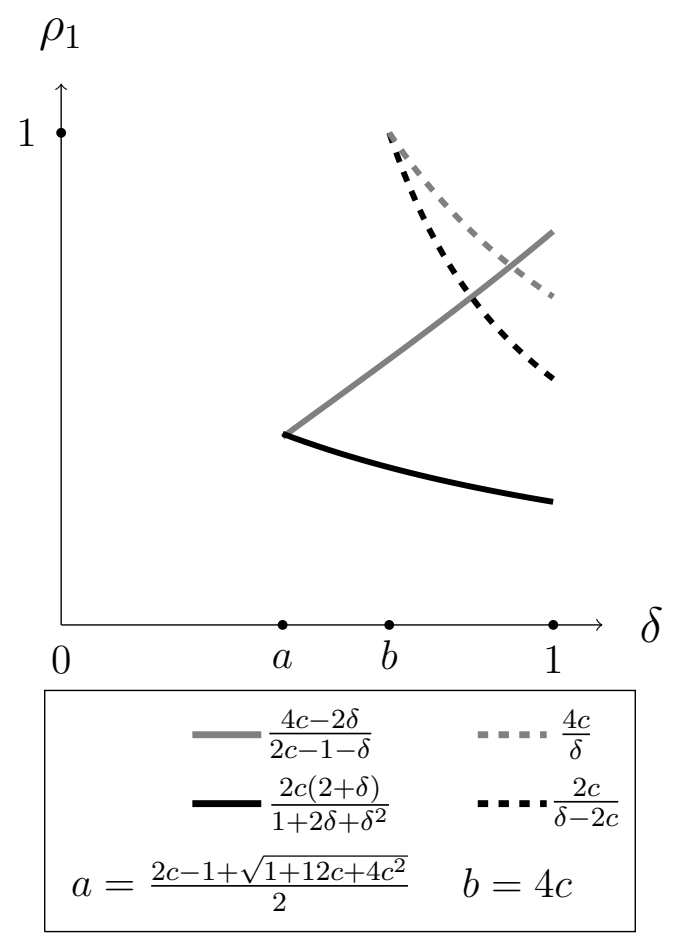

Figure 6: Deflated Ratings

Welfare Comparison. Since the strategic agency shirks at $t=2$ irrespective of the fee structure, and, for the symmetrical payoffs we consider, welfare does not depend on the 
agency's choice to inflate or deflate a rating in case it shirks, social welfare is therefore uniquely determined by the probability that the agency acquires information at $t=1$. Note that in the parameter region defined by $\delta \in\left(\frac{2 c-1+\sqrt{1+12 c+4 c^{2}}}{2}, 4 c\right)$ and $\rho_{1} \in\left(\frac{2 c(2+\delta)}{1+2 \delta+\delta^{2}}, \frac{4 c-2 \delta}{2 c-1-\delta}\right)$ the agency shirks at $t=1$ with upfront fees (Proposition 13) but acquires information with positive probability with contingent fees (Proposition 12), thus contingent fees improve expected social welfare. Note also that for $c$ sufficiently close to 0 any $\rho_{1}$ and $\delta$ satisfy $\delta>4 c$ and $\rho_{1}>\frac{4 c}{\delta}$, hence ensuring $e_{1}\left(\rho_{1}\right)=1$ with upfront fees (Proposition 13). As $e_{1}\left(\rho_{1}\right)<1$ everywhere with contingent fees (Proposition 12), the previous remarks establish that, for any $\rho_{1}$ and $\delta$, upfront fees improve expected social welfare relative to contingent fees as long as $c$ is sufficiently small.

Proof of Proposition 12: The function $\Phi$ is continuous, weakly increasing in each of its arguments, and $\Phi(1, \cdot, \cdot)=\Phi(\cdot, 1, \cdot)=1$. Notice that in any equilibrium the strategic agency must shirk and announce $r_{2}=1$ at $t=2$ with probability 1 giving $\pi_{2}=\Phi\left(\rho_{2}, 0,0\right)=\frac{\rho_{2}}{2-\rho_{2}}$. Define $V(\rho):=\frac{\rho}{2-\rho}$. Let $\rho_{1}^{+}(e, z)$ (respectively $\rho_{1}^{++}(e, z)$ ) denote the value of $\rho_{1}^{+}\left(\right.$respectively $\rho_{1}^{++}$) for $e_{1}\left(\rho_{1}\right)=e$ and $z_{1}\left(\rho_{1}\right)=z$. At $t=1$, the agency's expected intertemporal profit from shirking and announcing $r_{t}=1$ is equal to $\Phi\left(\rho_{1}, \hat{e}_{1}, \hat{z}_{1}\right)+\frac{\delta}{2} V\left(\rho_{1}^{+}\left(\hat{e}_{1}, \hat{z}_{1}\right)\right)$, the intertemporal profit from shirking and announcing $r_{t}=-1$ is equal to $\frac{\delta}{2} V\left(\rho_{1}^{++}\left(\hat{e}_{1}, \hat{z}_{1}\right)\right)$, and the intertemporal profit from acquiring information is equal to $\frac{1}{2} \Phi\left(\rho_{1}, \hat{e}_{1}, \hat{z}_{1}\right)-c+\frac{\delta}{2}\left[V\left(\rho_{1}^{+}\left(\hat{e}_{1}, \hat{z}_{1}\right)\right)+V\left(\rho_{1}^{++}\left(\hat{e}_{1}, \hat{z}_{1}\right)\right)\right]$. The rest of the proof contains 3 steps. Step 1 establishes than in equilibrium $e_{1}\left(\rho_{1}\right)<1$. Step 2 computes the probability with which the strategic agency must announce $r_{1}=-1$ if in equilibrium $e_{1}\left(\rho_{1}\right)=0$. Step 3 characterizes a parameter region in which $e_{1}\left(\rho_{1}\right)>0$.

Step 1: An equilibrium with $e_{1}\left(\rho_{1}\right)=1$ requires

$$
\frac{\delta V\left(\rho_{1}\right)}{2}-c \geq \frac{\Phi\left(\rho_{1}, 1, z_{1}\right)}{2},
$$

for some $z_{1}$. Note that, for any $z_{1}$ and any $\rho_{1}: \Phi\left(\rho_{1}, 1, z_{1}\right)=1>V\left(\rho_{1}\right)$. Thus (41) holds only if $\frac{\delta-1}{2}>c$. This condition is violated as $c>0>\frac{\delta-1}{2}$. Hence in equilibrium $e\left(\rho_{1}\right)<1$.

Step 2: Define $\tilde{z}\left(\rho_{1}\right)$ implicitly by

$$
\Phi\left(\rho_{1}, 0, \tilde{z}\left(\rho_{1}\right)\right)=\frac{\delta}{2}\left[V\left(\rho_{1}^{++}\left(0, \tilde{z}\left(\rho_{1}\right)\right)\right)-V\left(\rho_{1}^{+}\left(0, \tilde{z}\left(\rho_{1}\right)\right)\right)\right],
$$


which is equivalent to

$$
\frac{\rho_{1}}{1+\left(1-\rho_{1}\right)(1-2 \tilde{z})}=\frac{\delta}{2}\left(\frac{\rho_{1}}{\rho_{1}+2\left(1-\rho_{1}\right) \tilde{z}}-\frac{\rho_{1}}{1+\left(1-\rho_{1}\right)(1-2 \tilde{z})}\right) .
$$

Simplifying to solve for $\tilde{z}$ gives $\tilde{z}\left(\rho_{1}\right)=\frac{\delta\left(1-\rho_{1}\right)-\rho_{1}}{2\left(1-\rho_{1}\right)(\delta+1)}$. Note that $\tilde{z}\left(\rho_{1}\right)<1$ for all $\rho_{1} \in(0,1)$, while $\tilde{z}\left(\rho_{1}\right)>0$ if and only if $\rho_{1}<\frac{\delta}{1+\delta}$.

Now, in equilibrium, if $e_{1}\left(\rho_{1}\right)=0$ then $\Phi\left(\rho_{1}, 0, z_{1}\left(\rho_{1}\right)\right)>\frac{\delta}{2}\left[V\left(\rho_{1}^{++}\right)-V\left(\rho_{1}^{+}\right)\right]$implies $z_{1}\left(\rho_{1}\right)=0$ and $\Phi\left(\rho_{1}, 0, z\left(\rho_{1}\right)\right)<\frac{\delta}{2}\left[V\left(\rho_{1}^{++}\right)-V\left(\rho_{1}^{+}\right)\right]$implies $z\left(\rho_{1}\right)=1$. Thus, by construction of $\tilde{z}\left(\rho_{1}\right)$, in equilibrium, $e_{1}\left(\rho_{1}\right)=0$ implies $z\left(\rho_{1}\right)=\tilde{z}\left(\rho_{1}\right)$ if $\rho_{1} \leq \frac{\delta}{1+\delta}$ and $z\left(\rho_{1}\right)=0$ if $\rho_{1}>\frac{\delta}{1+\delta}$.

Step 3: Let $\bar{\rho}^{c}(\delta):=\frac{4 c-2 \delta}{2 c-1-\delta}$ and $\underline{\rho}^{c}(\delta)=\frac{2 c(2+\delta)}{1+2 \delta+\delta^{2}}$. Note that $\bar{\rho}^{c}(\delta) \in\left(\frac{\delta}{1+\delta}, 1\right) \Leftrightarrow \underline{\rho}^{c}(\delta) \in$ $\left(0, \frac{\delta}{1+\delta}\right) \Leftrightarrow \delta \in\left(\frac{2 c-1+\sqrt{1+12 c+4 c^{2}}}{2}, 1\right)$.

Fix $\delta \in\left(\frac{2 c-1+\sqrt{1+12 c+4 c^{2}}}{2}, 1\right)$. Consider a $\rho_{1} \geq \frac{\delta}{1+\delta}$. Step 2 ensures that an equilibrium with $e_{1}\left(\rho_{1}\right)=0$ exists if and only if: $\frac{\Phi\left(\rho_{1}, 0,0\right)}{2}+c \geq \frac{\delta V(1)}{2}$. This condition is equivalent to: $\rho_{1} \geq \bar{\rho}^{c}(\delta)$. Now consider a $\rho_{1} \leq \frac{\delta}{1+\delta}$. Step 2 ensures that an equilibrium with $e_{1}\left(\rho_{1}\right)=0$ exists if and only if: $\frac{\Phi\left(\rho_{1}, 0, \tilde{z}\left(\rho_{1}\right)\right)}{2}+c \geq \frac{\delta V\left(\rho_{1}^{++}\left(0, \tilde{z}\left(\rho_{1}\right)\right)\right)}{2}$. This condition is equivalent to: $\rho_{1} \leq \underline{\rho}^{c}(\delta)$. Thus, in light of step 1 , we conclude that for $\rho_{1} \in\left(\underline{\rho}^{c}(\delta), \bar{\rho}^{c}(\delta)\right)$ in equilibrium $e_{1}\left(\rho_{1}\right) \in(0,1)$.

Proof of Proposition 13: Note that $\Upsilon$ is continuous, weakly increasing in each of its arguments, and $\Upsilon(1, \cdot)=\Upsilon(\cdot, 1)=\frac{1}{2}$. Define $V(\rho):=\frac{\rho}{2}$. At $t=1$, the agency's expected intertemporal profit from shirking and announcing $r_{t}=1$ is equal to $\Upsilon\left(\rho_{1}, \hat{e}_{1}\right)+\frac{\delta}{2} V\left(\rho_{1}^{+}\right)$, the intertemporal profit from shirking and announcing $r_{t}=-1$ is equal to $\Upsilon\left(\rho_{1}, \hat{e}_{1}\right)+\frac{\delta}{2} V\left(\rho_{1}^{++}\right)$, and the intertemporal profit from acquiring information is equal to $\Upsilon\left(\rho_{1}, \hat{e}_{1}\right)+\frac{\delta}{2}\left[V\left(\rho_{1}^{+}\right)+V\left(\rho_{1}^{++}\right)\right]-c$. Observe that in any equilibrium $e_{1}\left(\rho_{1}\right)<1$ implies $z_{1}\left(\rho_{1}\right)=\frac{1}{2}$, since $z_{1}\left(\rho_{1}\right)>\frac{1}{2}$ (resp. $z_{1}\left(\rho_{1}\right)<$ $\left.\frac{1}{2}\right)$ implies $\rho_{1}^{+}>\rho_{1}^{++}\left(\operatorname{resp} . \rho_{1}^{+}<\rho_{1}^{++}\right)$and thus $V\left(\rho_{1}^{++}\right)<V\left(\rho_{1}^{+}\right)\left(\operatorname{resp} . V\left(\rho_{1}^{+}\right)<V\left(\rho_{1}^{++}\right)\right)$. Moreover, $e_{1}\left(\rho_{1}\right)=1$ implies $\rho_{1}^{+}=\rho_{1}^{++}=\rho_{1}$. We thus obtain $\rho_{1}^{+}=\rho_{1}^{++}=f\left(\rho_{1}, e_{1}\left(\rho_{1}\right)\right)$ in any equilibrium, where

$$
f\left(\rho_{1}, e_{1}\left(\rho_{1}\right)\right):=\frac{\rho_{1}}{\rho_{1}+\left(1-\rho_{1}\right)\left(e_{1}\left(\rho_{1}\right)+\frac{1}{2}\left(1-e_{1}\left(\rho_{1}\right)\right)\right)} .
$$

Next, an equilibrium in which $e_{1}\left(\rho_{1}\right)=0$ exists if and only if $\frac{\delta}{2} V\left(f\left(\rho_{1}, 0\right)\right) \leq c$ that is, if and 
only if either $\delta \leq 4 c$, or else $\delta>4 c$ and

$$
\rho_{1} \leq \frac{2 c}{\delta-2 c}
$$

Similarly, an equilibrium in which $e_{1}\left(\rho_{1}\right)=1$ exists if and only if $\frac{\delta}{2} V\left(f\left(\rho_{1}, 1\right)\right) \geq c$ that is, if and only if

$$
\rho_{1} \geq \frac{4 c}{\delta} .
$$

Note that $\frac{4 c}{\delta}<1 \Leftrightarrow \delta>4 c$.

An equilibrium with $e_{1}\left(\rho_{1}\right) \in(0,1)$ requires $\frac{\delta}{2} V\left(f\left(\rho_{1}, e_{1}\left(\rho_{1}\right)\right)\right)=c$. As $\frac{\partial f(\cdot)}{\partial e_{1}\left(\rho_{1}\right)}<0$, a (unique) equilibrium with $e_{1}\left(\rho_{1}\right) \in(0,1)$ exists if and only if $\delta>4 c$ and $\rho_{1} \in\left(\frac{2 c}{\delta-2 c}, \frac{4 c}{\delta}\right)$. 


\section{Online Appendix C}

We show in this appendix that our main results do not depend on the assumption that the agency obtains a fraction $\beta$ of all proceeds from selling projects to investors. Specifically, we generalize the baseline model by letting $\phi_{t}\left(r_{t}\right)$ satisfy

$$
\phi_{t}\left(r_{t}\right)= \begin{cases}f\left(\mathbb{E}\left[q_{t} \mid r_{t}=1, \rho_{t}, \hat{e}_{t}\right]\right) & \text { if } r_{t}=1 \\ 0 & \text { if } r_{t}=-1\end{cases}
$$

where $f(\cdot):[0,1] \rightarrow[0,1]$ denotes a strictly increasing continuous function satisfying $f(x) \leq x$ for all $x \in[0,1]$. We assume in line with the baseline model that $c<\frac{f(1)}{2}$. In what follows we first state the main results, and then provide all the proofs.

Proposition 14. An equilibrium exists and is unique. In equilibrium, $e(0)=0$; for $\rho>0$ the equilibrium is characterized by cutoffs $\underline{\rho}$ and $\bar{\rho}, \underline{\rho} \leq \bar{\rho}$, such that

$$
\left\{\begin{array}{l}
\text { if } \quad \rho \in[\bar{\rho}, 1] \quad \text { then } \quad e(\rho)=0, \\
\text { if } \rho \in(\underline{\rho}, \bar{\rho}) \quad \text { then } \quad e(\rho) \in(0,1), \\
\text { if } \rho \in(0, \underline{\rho}] \quad \text { then } \quad e(\rho)=1 .
\end{array}\right.
$$

Moreover, the equilibrium fee is a non-decreasing function of the agency's reputation.

The model with upfront fees is generalized by letting

$$
\phi_{t}(1)=\phi_{t}(-1)=f\left(\mathbb{P}\left(r_{t}=1 \mid \rho_{t}, \hat{e}_{t}\right) \mathbb{E}\left[q_{t} \mid r_{t}=1, \rho_{t}, \hat{e}_{t}\right]\right)
$$

Proposition 15. If $\delta<\frac{2 c}{f\left(\frac{1}{2}\right)+c}$ then $e(\rho)=0$ for all $\rho \in[0,1]$ is the unique equilibrium with upfront fees. If $\delta>\frac{2 c}{f\left(\frac{1}{2}\right)+c}$ the unique equilibrium is

$$
e(\rho)=\left\{\begin{array}{l}
1 \text { if } \rho>0 \\
0 \text { if } \rho=0
\end{array}\right.
$$

Combining Propositions 14 and 15 yields the next theorem.

Theorem 3. There exists $\tilde{\rho}$ such that, if $\delta \in\left(\frac{2 c}{f(1)+c}, \frac{2 c}{f\left(\frac{1}{2}\right)+c}\right)$ then, for $\rho_{1} \in(0, \tilde{\rho})$, contingent fees improve expected social welfare relative to upfront fees. Moreover if $\frac{2 c}{f\left(\frac{1}{2}\right)+c}<\frac{2 f(1)+4 c}{3 f(1)+2 c}$ then 
for $\delta \in\left(\frac{2 c}{f\left(\frac{1}{2}\right)+c}, \frac{2 f(1)+4 c}{3 f(1)+2 c}\right)$ upfront fees improve expected social welfare relative to contingent fees. In all other cases, expected social welfare is the same whether fees are upfront or contingent.

We prove in the rest of this appendix all of the previous results. Define $\Phi(\cdot, \cdot):[0,1] \times$ $[0,1] \rightarrow \mathbb{R}$ by

$$
\Phi(\rho, e):=\frac{1-(1-\rho)(1-e)}{1+(1-\rho)(1-e)} .
$$

Given a function $e:[0,1] \rightarrow[0,1]$, define

$$
\rho^{+}:= \begin{cases}0 & \text { if } \rho=0 \\ \frac{\rho}{\rho+(1-\rho) e(\rho)} & \text { if } \rho>0\end{cases}
$$

Lemma 7. In any equilibrium, $e(0)=0$ and $V(0)=0$, where $V(\cdot)$ denotes the equilibrium value function. For all $\rho>0$,

$$
\left\{\begin{array}{l}
\text { if } \quad \frac{\delta}{2} V\left(\rho^{+}\right)>\frac{1}{2} f(\Phi(\rho, e(\rho)))+c \text { then } \quad e(\rho)=1 \\
\text { if } \quad \frac{\delta}{2} V\left(\rho^{+}\right)<\frac{1}{2} f(\Phi(\rho, e(\rho)))+c \text { then } \quad e(\rho)=0
\end{array}\right.
$$

Proof: By virtue of Bellman's Principle of Optimality,

$$
V(\rho)=\max \left\{f(\Phi(\rho, e(\rho)))+\delta\left(\frac{1}{2} V(\rho)+\frac{1}{2} V(0)\right), \frac{1}{2} f(\Phi(\rho, e(\rho)))-c+\delta\left(\frac{1}{2} V(\rho)+\frac{1}{2} V\left(\rho^{+}\right)\right)\right\}
$$

for all $\rho \in[0,1]$, and the choice implied by $e(\rho)$ maximizes the right-hand side of the expression above. That is:

$$
\left\{\begin{array}{l}
e(\rho)=1 \quad \text { if } \quad \frac{1}{2} f(\Phi(\rho, e(\rho)))-c+\delta\left(\frac{1}{2} V(\rho)+\frac{1}{2} V\left(\rho^{+}\right)\right)>f(\Phi(\rho, e(\rho)))+\delta\left(\frac{1}{2} V(\rho)+\frac{1}{2} V(0)\right) \\
e(\rho)=0 \quad \text { if } \quad \frac{1}{2} f(\Phi(\rho, e(\rho)))-c+\delta\left(\frac{1}{2} V(\rho)+\frac{1}{2} V\left(\rho^{+}\right)\right)<f(\Phi(\rho, e(\rho)))+\delta\left(\frac{1}{2} V(\rho)+\frac{1}{2} V(0)\right)
\end{array}\right.
$$

We are only left to show that $e(0)=0$ and $V(0)=0$. That $e(0)=0$ follows from the above, noting that $\rho^{+}=0$ if $\rho=0$. Substituting back into (42) then yields $V(0)=f(\Phi(0,0))+$ $\delta V(0)=\delta V(0)$. Hence $V(0)=0$. 
Lemma 8. In any equilibrium,

$$
\left\{\begin{array}{l}
\text { if } e(\rho)=1 \text { then } V(\rho)=\frac{\frac{1}{2} f(\Phi(\rho, 1))-c}{1-\delta}=\max \left\{\frac{\frac{1}{2} f(\Phi(\rho, 1))-c}{1-\delta}, \frac{f(\Phi(\rho, 1))}{1-\frac{\delta}{2}}\right\} \\
\text { if } e(\rho)<1 \text { then } V(\rho)=\frac{f(\Phi(\rho, e(\rho)))}{1-\frac{\delta}{2}}
\end{array}\right.
$$

Furthermore, $V(1)=\max \left\{\frac{\frac{1}{2} f(\Phi(1, e(1)))-c}{1-\delta}, \frac{f(\Phi(1, e(1)))}{1-\frac{\delta}{2}}\right\} \geq V(\rho)$, for all $\rho \in[0,1]$.

Proof: The lemma follows from Bellman's Principle of Optimality together with the observations that (a) $e(\rho)=1$ implies $\rho^{+}=\rho$, (b) $\rho=1$ implies $\rho^{+}=1$, (c) $\Phi(1, e)=1$ for all $e \in[0,1]$, and $(\mathrm{d}) \Phi(\cdot, \cdot)$ is weakly increasing in both variables.

Proposition 16. If $\delta \geq \bar{\delta}:=\frac{2 f(1)+4 c}{3(1)+2 c}$ then

$$
e(\rho)=\left\{\begin{array}{l}
1 \text { if } \rho>0 \\
0 \text { if } \rho=0
\end{array}\right.
$$

is an equilibrium. If $\delta<\bar{\delta}$, in any equilibrium: $e(\rho)<1$ for all $\rho \in[0,1]$.

Proof: By Lemma 7, $e(0)=0$ for all $\delta$. Next, consider $\rho>0$. If in equilibrium $e(\rho)=1$ then by Lemma 7 and the observation that $\rho^{+}=\rho$ :

$$
\frac{\delta}{2} V(\rho) \geq \frac{1}{2} f(\Phi(\rho, 1))+c
$$

Applying Lemma 8 now yields

$$
\frac{\delta}{2}\left(\frac{\frac{1}{2} f(\Phi(\rho, 1))-c}{1-\delta}\right) \geq \frac{1}{2} f(\Phi(\rho, 1))+c,
$$

or, equivalently, $\delta \geq \bar{\delta}$ once we note that $\Phi(\rho, 1)=1$. The condition $\delta \geq \bar{\delta}$ is thus necessary for $e(\rho)=1$. Sufficiency follows from the one-shot deviation principle.

Lemma 9. The following are equivalent:

$$
\delta \geq \bar{\delta}
$$




$$
\begin{gathered}
\frac{1}{2} f(\Phi(\rho, 1))+c \leq \frac{\delta}{2}\left(\frac{\frac{1}{2} f(\Phi(\rho, 1))-c}{1-\delta}\right) \\
\frac{1}{2} f(\Phi(\rho, 1))+c \leq \frac{\delta}{2}\left(\frac{f(\Phi(\rho, 1))}{1-\frac{\delta}{2}}\right) \\
\min \left\{\frac{\frac{1}{2} f(\Phi(\rho, 1))-c}{1-\delta}, \frac{f(\Phi(\rho, 1))}{1-\frac{\delta}{2}}\right\}=\frac{f(\Phi(\rho, 1))}{1-\frac{\delta}{2}}
\end{gathered}
$$

Moreover, the equivalence between (44)-(46) continues to hold with strict inequalities instead of weak inequalities.

Proof: Equivalence is easily checked using $\Phi(\rho, 1)=1$.

Proposition 17. If $\delta>\bar{\delta}$ then (43) is the unique equilibrium.

Proof: By Lemma $7, e(0)=0$ in any equilibrium. So we are only left to show that, in any equilibrium, $e(\rho)=1$ for all $\rho>0$.

Suppose that an equilibrium exists such that $e(\hat{\rho})<1$ for some $\hat{\rho}>0$. Applying first Lemma 7 then Lemma 8:

$$
\frac{1}{2} f(\Phi(\hat{\rho}, e(\hat{\rho})))+c \geq \frac{\delta}{2} V\left(\hat{\rho}^{+}\right) \geq \frac{\delta}{2}\left(\frac{f\left(\Phi\left(\hat{\rho}^{+}, e\left(\hat{\rho}^{+}\right)\right)\right)}{1-\frac{\delta}{2}}\right) .
$$

We thus obtain, using the equivalence between (44) and (46) (with strict inequalities), the following sequence of inequalities:

$$
\frac{\delta}{2}\left(\frac{f(\Phi(\hat{\rho}, 1))}{1-\frac{\delta}{2}}\right)>\frac{1}{2} f(\Phi(\hat{\rho}, 1))+c \geq \frac{1}{2} f(\Phi(\hat{\rho}, e(\hat{\rho})))+c \geq \frac{\delta}{2}\left(\frac{f\left(\Phi\left(\hat{\rho}^{+}, e\left(\hat{\rho}^{+}\right)\right)\right)}{1-\frac{\delta}{2}}\right)
$$

from which we infer that $e\left(\hat{\rho}^{+}\right)<1$. We can thus repeat the steps above with $\hat{\rho}^{+}$instead of $\hat{\rho}$, and so on. This process determines a sequence $\left\{\rho_{n}\right\}$ such that, for all $n$ :

(i) $e\left(\rho_{n}\right)<1$

(ii) $\rho_{n+1}=\frac{\rho_{n}}{\rho_{n}+\left(1-\rho_{n}\right) e\left(\rho_{n}\right)}>\rho_{n}$,

(iii) $\frac{1}{2} f\left(\Phi\left(\rho_{n}, e\left(\rho_{n}\right)\right)\right)+c \geq \frac{\delta}{2}\left(\frac{f\left(\Phi\left(\rho_{n+1}, e\left(\rho_{n+1}\right)\right)\right)}{1-\frac{\delta}{2}}\right)$. 
By (i)-(ii), either $e\left(\rho_{n}\right) \rightarrow 1$ or $\rho_{n} \rightarrow 1$. Hence, taking limits in (iii) yields (using continuity of $\Phi(\cdot, \cdot)$, continuity of $f(\cdot)$ and the fact that $\Phi(1, e)=\Phi(\rho, 1)=1$ for all $e$ and $\rho$ in $[0,1])$ :

$$
\frac{1}{2} f(\Phi(1,1))+c \geq \frac{\delta}{2}\left(\frac{f(\Phi(1,1))}{1-\frac{\delta}{2}}\right) .
$$

The equivalence between (44) and (46) (with strict inequalities) establishes a contradiction between (48) and $\delta>\bar{\delta}$.

Proposition 18. If $\delta \leq \underline{\delta}:=\frac{2 c}{f(1)+c}$ then $e(\rho)=0$ for all $\rho \in[0,1]$ is the unique equilibrium. Proof: Note first that $\delta \leq \underline{\delta}$ if and only if

$$
c \geq \frac{\delta}{2}\left(\frac{f(\Phi(1, e(1)))}{1-\frac{\delta}{2}}\right) .
$$

Next, the assumption $f(1)>2 c>0$ implies $\underline{\delta}<\bar{\delta}$; combining Lemmas 8 and 9 thus shows that, in any equilibrium,

$$
V(1)=\frac{f(\Phi(1, e(1)))}{1-\frac{\delta}{2}}
$$

whenever $\delta<\underline{\delta}$. Combining (49), (50) and Lemma 8 now yields $c>\frac{\delta}{2} V(\rho)$, for all $\rho \in[0,1)$. Hence, by Lemma $7, e(\rho)=0$, for all $\rho \in[0,1]$.

That $e(\rho)=0$ for all $\rho \in[0,1]$ is an equilibrium is immediate from (49), (50), and the one-shot deviation principle.

Proposition 19. Let $\delta \in(\underline{\delta}, \bar{\delta})$. There exists a unique equilibrium. In equilibrium,

$$
\begin{cases}e(\rho)=0 & \text { if } \rho \in\{0\} \cup[\tilde{\rho}, 1] \\ e(\rho) \in(0, \tilde{e}] & \text { if } \rho \in(0, \tilde{\rho})\end{cases}
$$

where $\tilde{\rho} \in(0,1)$ and $\tilde{e} \in(0,1)$ are defined implicitly by

$$
\frac{1}{2} f(\Phi(\tilde{\rho}, 0))+c=\frac{\delta}{2}\left(\frac{f(\Phi(1,0))}{1-\frac{\delta}{2}}\right),
$$


and

$$
\frac{1}{2} f(\Phi(0, \tilde{e}))+c=\frac{\delta}{2}\left(\frac{f(\Phi(0,1))}{1-\frac{\delta}{2}}\right)
$$

respectively.

Proof: Applying Lemma 9,

$$
\delta<\bar{\delta} \Leftrightarrow \frac{1}{2} f(\Phi(\rho, 1))+c>\frac{\delta}{2}\left(\frac{f(\Phi(\rho, 1))}{1-\frac{\delta}{2}}\right)
$$

Moreover, by (49),

$$
\delta>\underline{\delta} \Leftrightarrow c<\frac{\delta}{2}\left(\frac{f(\Phi(\rho, 1))}{1-\frac{\delta}{2}}\right) .
$$

Thus $\tilde{\rho} \in(0,1)$ and $\tilde{e} \in(0,1)$.

We now prove the rest of the proposition. We will first proceed by induction to show that there can exist at most one equilibrium. We will then argue that the inductive procedure yields an equilibrium. As a preliminary step, observe that by Lemma 8 and the equivalence between (44) and (47), an equilibrium must satisfy:

$$
V(\rho)=\frac{f(\Phi(\rho, e(\rho)))}{1-\frac{\delta}{2}}
$$

for all $\rho \in[0,1]$.

The inductive procedure starts as follows. Combining (52) and (54), any equilibrium must be such that, for all $\rho>\tilde{\rho}$ :

$$
\frac{1}{2} f(\Phi(\rho, 0))+c>\frac{\delta}{2} V\left(\rho^{+}\right)
$$

Thus, by Lemma 7, if an equilibrium exists it must satisfy $e(\rho)=0$ for all $\rho>\tilde{\rho}$. A similar argument shows that in fact the same must be true for $\rho=\tilde{\rho}$.

By contrast, consider $\rho \in(0, \tilde{\rho})$. The combination of $(52),(54)$, and Lemma 7 shows that $e(\rho)=0$ is impossible in equilibrium. Similarly, the combination of (53), (54) and Lemma 7 shows that $e(\rho)>\tilde{e}$ is impossible in equilibrium. Thus, any equilibrium must satisfy (51). By virtue of Lemma 7 this in turn implies that the indifference condition

$$
\frac{\delta}{2} V\left(\rho^{+}\right)=\frac{1}{2} f(\Phi(\rho, e(\rho)))+c
$$

must hold for all $\rho \in(0, \tilde{\rho})$. 
Next define $\rho_{1}<\tilde{\rho}$ such that

$$
\tilde{\rho}=\frac{\rho_{1}}{\rho_{1}+\left(1-\rho_{1}\right) \tilde{e}} .
$$

By construction of $\rho_{1}$ and property (51), in any equilibrium: $\rho^{+} \geq \tilde{\rho}$ for all $\rho \in\left[\rho_{1}, \tilde{\rho}\right)$. (55), (51) and (54) now pin down a unique candidate equilibrium $e(\rho)$ for each $\rho \in\left[\rho_{1}, \tilde{\rho}\right)$ (which moreover is continuous in $\rho$ ). Repeating the step above with $\rho_{1}$ instead of $\tilde{\rho}$ yields $\rho_{2}<\rho_{1}$ and a unique candidate equilibrium $e(\rho)$ for each $\rho \in\left[\rho_{2}, \rho_{1}\right)$, and so on. This defines a sequence $\left\{\rho_{n}\right\}$ where, for all $n, \tilde{\rho}_{n}=\frac{\rho_{n+1}}{\rho_{n+1}+\left(1-\rho_{n+1}\right) \tilde{e}}$. As $\tilde{e}<1, \rho_{n} \rightarrow 0$. This inductive procedure therefore pins down a unique candidate equilibrium. That this candidate equilibrium is in fact an equilibrium is a consequence of the one-shot deviation principle.

Proof of Proposition 14: Follows from Propositions 16-19.

Define $\Upsilon(\cdot, \cdot):[0,1] \times[0,1] \rightarrow \mathbb{R}$ by

$$
\Upsilon(\rho, e):=\frac{1}{2}(1-(1-\rho)(1-e)) .
$$

Lemma 10. In any equilibrium, $e(0)=0$ and $V(0)=0$, where $V(\cdot)$ denotes the equilibrium value function. For all $\rho>0$,

$$
\begin{cases}\text { if } & \frac{\delta}{2} V\left(\rho^{+}\right)>c \text { then } \quad e(\rho)=1 \\ \text { if } & \frac{\delta}{2} V\left(\rho^{+}\right)<c \text { then } \quad e(\rho)=0 .\end{cases}
$$

Proof: By virtue of Bellman's Principle of Optimality,

$$
V(\rho)=\max \left\{f(\Upsilon(\rho, e(\rho)))+\delta\left(\frac{1}{2} V(\rho)+\frac{1}{2} V(0)\right), f(\Upsilon(\rho, e(\rho)))-c+\delta\left(\frac{1}{2} V(\rho)+\frac{1}{2} V\left(\rho^{+}\right)\right)\right\},
$$

for all $\rho \in[0,1]$, and the choice implied by $e(\rho)$ maximizes the right-hand side of $(56)$. That is:

$$
\begin{cases}e(\rho)=1 & \text { if } \quad f(\Upsilon(\rho, e(\rho)))-c+\delta\left(\frac{1}{2} V(\rho)+\frac{1}{2} V\left(\rho^{+}\right)\right)>f(\Upsilon(\rho, e(\rho)))+\delta\left(\frac{1}{2} V(\rho)+\frac{1}{2} V(0)\right), \\ e(\rho)=0 & \text { if } \quad f(\Upsilon(\rho, e(\rho)))-c+\delta\left(\frac{1}{2} V(\rho)+\frac{1}{2} V\left(\rho^{+}\right)\right)<f(\Upsilon(\rho, e(\rho)))+\delta\left(\frac{1}{2} V(\rho)+\frac{1}{2} V(0)\right) .\end{cases}
$$


We are only left to show that $e(0)=0$ and $V(0)=0$. That $e(0)=0$ follows from the above, noting that $\rho^{+}=0$ if $\rho=0$. Substituting back into (56) then yields $V(0)=f(\Phi(0,0))+$ $\delta V(0)=\delta V(0)$. Hence $V(0)=0$.

Lemma 11. In any equilibrium,

$$
\left\{\begin{array}{l}
\text { if } e(\rho)=1 \text { then } V(\rho)=\frac{f(\Upsilon(\rho, 1))-c}{1-\delta}=\max \left\{\frac{f(\Upsilon(\rho, 1))-c}{1-\delta}, \frac{f(\Upsilon(\rho, 1))}{1-\frac{\delta}{2}}\right\}, \\
\text { if } e(\rho)<1 \text { then } V(\rho)=\frac{f(\Upsilon(\rho, e(\rho)))}{1-\frac{\delta}{2}}
\end{array}\right.
$$

Furthermore, $V(1)=\max \left\{\frac{f(\Upsilon(1, e(1)))-c}{1-\delta}, \frac{f(\Upsilon(1, e(1)))}{1-\frac{\delta}{2}}\right\} \geq V(\rho)$, for all $\rho \in[0,1]$.

Proof: The lemma follows from Bellman's Principle of Optimality together with the observations that (a) $e(\rho)=1$ implies $\rho^{+}=\rho$, (b) $\rho=1$ implies $\rho^{+}=1$, (c) $\Upsilon(1, e)=\frac{1}{2}$ for all $e \in[0,1]$, and $(\mathrm{d}) \Upsilon(\cdot, \cdot)$ is weakly increasing in both variables.

Proposition 20. If $\delta>\frac{2 c}{f\left(\frac{1}{2}\right)+c}$ then

$$
e(\rho)=\left\{\begin{array}{l}
1 \text { if } \rho>0, \\
0 \text { if } \rho=0 .
\end{array}\right.
$$

is an equilibrium. If $\delta<\frac{c}{f\left(\frac{1}{2}\right)+c}$, in any equilibrium: $e(\rho)<1$ for all $\rho \in[0,1]$.

Proof: By Lemma 10, $e(0)=0$ for all $\delta$. Next, consider $\rho>0$. If in equilibrium $e(\rho)=1$ then Lemma 10 and the observation that $\rho^{+}=\rho$ yield

$$
\frac{\delta}{2} V(\rho) \geq c
$$

Applying Lemma 11 now gives

$$
\frac{\delta}{2}\left(\frac{f(\Upsilon(\rho, 1))-c}{1-\delta}\right) \geq c
$$

or, equivalently, $\delta \geq \frac{2 c}{f\left(\frac{1}{2}\right)+c}$ once we note that $\Upsilon(\rho, 1)=\frac{1}{2}$. The condition $\delta \geq \frac{2 c}{f\left(\frac{1}{2}\right)+c}$ is thus necessary for $e(\rho)=1$. Sufficiency follows from the one-shot deviation principle. 
Lemma 12. The following are equivalent:

$$
\begin{gathered}
\delta \geq \frac{2 c}{f\left(\frac{1}{2}\right)+c}, \\
c \leq \frac{\delta}{2}\left(\frac{f(\Upsilon(\rho, 1))-c}{1-\delta}\right), \\
c \leq \frac{\delta}{2}\left(\frac{f(\Upsilon(\rho, 1))}{1-\frac{\delta}{2}}\right), \\
\min \left\{\frac{f(\Upsilon(\rho, 1))-c}{1-\delta}, \frac{f(\Upsilon(\rho, 1))}{1-\frac{\delta}{2}}\right\}=\frac{f(\Upsilon(\rho, 1))}{1-\frac{\delta}{2}} .
\end{gathered}
$$

The equivalence between (58)-(60) continues to hold with strict inequalities instead of weak inequalities.

Proof: Equivalence is easily checked using $\Upsilon(\rho, 1)=\frac{1}{2}$.

Proposition 21. If $\delta>\frac{2 c}{f\left(\frac{1}{2}\right)+c}$ then (57) is the unique equilibrium.

Proof: By Lemma 10, $e(0)=0$ in any equilibrium. So we are only left to show that, in any equilibrium, $e(\rho)=1$ for all $\rho>0$.

Suppose an equilibrium exists such that $e(\hat{\rho})<1$ for some $\hat{\rho}>0$. Applying first Lemma 10 then Lemma 11:

$$
c \geq \frac{\delta}{2} V\left(\hat{\rho}^{+}\right) \geq \frac{\delta}{2}\left(\frac{f\left(\Upsilon\left(\hat{\rho}^{+}, e\left(\hat{\rho}^{+}\right)\right)\right)}{1-\frac{\delta}{2}}\right) .
$$

We thus obtain, using equivalence of (58) and (60) (with strict inequalities), the following sequence of inequalities:

$$
\frac{\delta}{2}\left(\frac{f(\Upsilon(\hat{\rho}, 1))}{1-\frac{\delta}{2}}\right)>c \geq \frac{\delta}{2}\left(\frac{f\left(\Upsilon\left(\hat{\rho}^{+}, e\left(\hat{\rho}^{+}\right)\right)\right)}{1-\frac{\delta}{2}}\right)
$$

from which we infer that $e\left(\hat{\rho}^{+}\right)<1$. We can thus repeat the steps above with $\hat{\rho}^{+}$instead of $\hat{\rho}$, and so on. This process determines a sequence $\left\{\rho_{n}\right\}$ such that, for all $n$ :

(i) $e\left(\rho_{n}\right)<1$,

(ii) $\rho_{n+1}=\frac{\rho_{n}}{\rho_{n}+\left(1-\rho_{n}\right) e\left(\rho_{n}\right)}>\rho_{n}$, 
(iii) $c \geq \frac{\delta}{2}\left(\frac{f\left(\Upsilon\left(\rho_{n+1}, e\left(\rho_{n+1}\right)\right)\right)}{1-\frac{\delta}{2}}\right)$.

By (i)-(ii), either $e\left(\rho_{n}\right) \rightarrow 1$ or $\rho_{n} \rightarrow 1$. Hence, taking limits in (iii) yields (using continuity of $\Upsilon(\cdot, \cdot)$ and the fact that $\Upsilon(1, e)=\Upsilon(\rho, 1)=\frac{1}{2}$ for all $e$ and $\rho$ in $\left.[0,1]\right)$ :

$$
c \geq \frac{\delta}{2}\left(\frac{f(\Upsilon(1,1))}{1-\frac{\delta}{2}}\right) .
$$

The equivalence between (58) and (60) (with strict inequalities) establishes a contradiction with (62).

Proposition 22. If $\delta<\frac{2 c}{f\left(\frac{1}{2}\right)+c}$ then $e(\rho)=0$ for all $\rho \in[0,1]$ is the unique equilibrium.

Proof: By Lemma 12, we have $\delta<\frac{2 c}{f\left(\frac{1}{2}\right)+c}$ if and only if

$$
c>\frac{\delta}{2}\left(\frac{f(\Upsilon(1, e(1)))}{1-\frac{\delta}{2}}\right) .
$$

Next, combining Lemmas 11 and 12 yields

$$
V(1)=\frac{f(\Upsilon(1, e(1)))}{1-\frac{\delta}{2}}
$$

Combining (63) and (64) gives $c>\frac{\delta}{2} V(1)$; hence, by Lemma 11, $c>\frac{\delta}{2} V(\rho)$ for all $\rho \in[0,1]$. Lemma 10 thus yields $e(\rho)=0$, for all $\rho \in[0,1]$.

That $e(\rho)=0$, for all $\rho \in[0,1]$ is an equilibrium is immediate from (63), (64), and the one-shot deviation principle.

Proof of Proposition 15: Follows from Propositions 20-22.

Proof of Theorem 3: Proposition 14 characterizes the unique equilibrium with contingent fees, and Proposition 15 characterizes the unique equilibrium with upfront fees. The cutoff $\bar{\delta}=\frac{2 f(1)+4 c}{3 f(1)+2 c}$ is taken from Proposition 16. The cutoff $\underline{\delta}=\frac{2 c}{f(1)+c}$ is taken from Proposition 18. With upfront fees, either the strategic agency shirks irrespective of $\rho_{t}$ or the strategic agency acquires information with probability 1 irrespective of $\rho_{t}$. Hence, all that remains to show is 
that the expected period- $t$ welfare is an increasing function of the probability with which the strategic agency chooses to acquire information. For this proof

The expected period- $t$ welfare is

$$
\begin{aligned}
\mathbb{P}\left(r_{t}=1\right) \mathbb{E}\left[q_{t} \mid r_{t}=1\right]-c\left(\rho_{t}+\left(1-\rho_{t}\right) e\left(\rho_{t}\right)\right)= & \left(\frac{1}{2} \cdot 1+\frac{1}{2}\left(1-\rho_{t}\right)\left(1-e\left(\rho_{t}\right)\right)\right) \frac{1-\left(1-\rho_{t}\right)\left(1-e\left(\rho_{t}\right)\right)}{1+\left(1-\rho_{t}\right)\left(1-e\left(\rho_{t}\right)\right)} \\
& -c\left(\rho_{t}+\left(1-\rho_{t}\right) e\left(\rho_{t}\right)\right) \\
= & \left(\frac{1}{2}-c\right)\left(\rho_{t}+\left(1-\rho_{t}\right) e\left(\rho_{t}\right)\right)
\end{aligned}
$$

the result follows, since $c<\frac{1}{2}$. 


\section{Online Appendix D}

In this appendix we generalize the model presented in Online Appendix B by letting the prior probability $\gamma$ of $q_{t}=1$ take any value in $(0,1)$ and show that all our main results continue to hold. We normalize a project's return so that either $q_{t}=1$ or $q_{t}=\frac{-\gamma}{1-\gamma}$. Hence $\mathbb{E}\left[q_{t}\right]=0$ irrespective of $\gamma$. The model described in Online Appendix B corresponds to the case $\gamma=\frac{1}{2}$. The definitions from Online Appendix B all apply here as well with the functions $\Phi$ and $\Upsilon$ now replaced, respectively, by

$$
\Phi^{\dagger}\left(\rho_{t}, \hat{e}_{t}, \hat{z}_{t}\right):=\frac{1-\left(1-\rho_{t}\right)\left(1-\hat{e}_{t}\right)}{1+\frac{1-\gamma}{\gamma}\left(1-\rho_{t}\right)\left(1-\hat{e}_{t}\right)\left(1-\frac{\hat{z}_{t}}{1-\gamma}\right)}
$$

and

$$
\Upsilon^{\dagger}\left(\rho_{t}, \hat{e}_{t}\right):=\gamma\left[1-\left(1-\rho_{t}\right)\left(1-\hat{e}_{t}\right)\right]
$$

The Equilibria. We next characterize the equilibria with, respectively, contingent fees and upfront fees. With 2 periods the strategic agency always shirks in period 2. So the focus is on information acquisition at $t=1$. Proofs of the propositions are relegated to the end of this appendix.

Proposition 23. Let fees be contingent. In equilibrium $e_{1}\left(\rho_{1}\right)<1$ for all $\rho_{1} \in(0,1)$. Moreover, the equilibrium is characterized by cutoffs $\bar{\rho}^{\dagger}, \underline{\rho}^{\dagger}$ and $\underline{\delta}$ such that, for $\delta>\underline{\delta}, e_{1}\left(\rho_{1}\right) \in(0,1)$ for all $\rho_{1} \in\left(\underline{\rho}^{\dagger}, \bar{\rho}^{\dagger}\right)$.

Proposition 24. Let fees be upfront. Let $x:=\min \{\gamma, 1-\gamma\}$. In equilibrium, if $\delta \leq \frac{c}{\gamma x}$ then $e_{1}\left(\rho_{1}\right)=0$ for all $\rho_{1} \in(0,1)$. If instead $\delta>\frac{c}{\gamma x}$ then

$$
\left\{\begin{aligned}
\rho_{1} & \in\left(\frac{c}{\delta \gamma x}, 1\right) \quad \text { implies } & e\left(\rho_{1}\right)=1 ; \\
\rho_{1} & \in\left(\frac{c}{\delta \gamma-c}, \frac{c}{\delta \gamma x}\right) \quad \text { implies } & e\left(\rho_{1}\right) \in(0,1) ; \\
\rho_{1} & \in\left(0, \frac{c}{\delta \gamma-c}\right) \quad \text { implies } & e\left(\rho_{1}\right)=0 .
\end{aligned}\right.
$$

Welfare Comparison. As in Online Appendix B, since the strategic agency shirks at $t=2$ irrespective of the fee structure, and, for the payoffs we consider, welfare does not depend on the agency's choice to inflate or deflate a rating in case it shirks, social welfare is uniquely determined by the probability that the agency acquires information at $t=1$. In the parameter 
region defined by $\delta \in\left(\underline{\delta}, \frac{c}{\gamma x}\right)$ and $\rho_{1} \in\left(\underline{\rho}^{\dagger}, \bar{\rho}^{\dagger}\right)$ the agency shirks at $t=1$ with upfront fees (Proposition 24) but acquires information with positive probability with contingent fees (Proposition 23), thus contingent fees improve expected social welfare. Since $\underline{\delta}<\frac{c}{\gamma x}$, the aforementioned region is non-empty (we call this observation Remark 1 and prove it below). Note too that, for $c$ sufficiently close to 0 , any $\rho_{1}$ and $\delta$ satisfy $\delta>\frac{c}{\gamma x}$ and $\rho_{1}>\frac{c}{\delta \gamma x}$, hence ensuring $e_{1}\left(\rho_{1}\right)=1$ with upfront fees (Proposition 24). As $e_{1}\left(\rho_{1}\right)<1$ everywhere with contingent fees (Proposition 23), then for any $\rho_{1}$ and $\delta$ upfront fees improve expected social welfare for sufficiently small $c$.

Proof of Proposition 23: The function $\Phi^{\dagger}$ is continuous, weakly increasing in each of its arguments, and $\Phi^{\dagger}(1, \cdot, \cdot)=\Phi^{\dagger}(\cdot, 1, \cdot)=1$. Notice that in any equilibrium the strategic agency must shirk and announce $r_{2}=1$ at $t=2$ with probability 1 giving $\pi_{2}=$ $\Phi^{\dagger}\left(\rho_{2}, 0,0\right)=\frac{\gamma \rho_{2}}{1-(1-\gamma) \rho_{2}}$. Define $V(\rho):=\frac{\gamma \rho}{1-(1-\gamma) \rho}$. Let $\rho_{1}^{+}(e, z)$ (respectively $\left.\rho_{1}^{++}(e, z)\right)$ denote the value of $\rho_{1}^{+}$(respectively $\rho_{1}^{++}$) for $e_{1}\left(\rho_{1}\right)=e$ and $z_{1}\left(\rho_{1}\right)=z$. At $t=1$, the agency's expected intertemporal profit from shirking and announcing $r_{t}=1$ is equal to $\Phi^{\dagger}\left(\rho_{1}, \hat{e}_{1}, \hat{z}_{1}\right)+\delta \gamma V\left(\rho_{1}^{+}\left(\hat{e}_{1}, \hat{z}_{1}\right)\right)$, the intertemporal profit from shirking and announcing $r_{t}=-1$ is equal to $\delta(1-\gamma) V\left(\rho_{1}^{++}\left(\hat{e}_{1}, \hat{z}_{1}\right)\right)$, and the intertemporal profit from acquiring information is equal to $\gamma \Phi^{\dagger}\left(\rho_{1}, \hat{e}_{1}, \hat{z}_{1}\right)-c+\delta\left[\gamma V\left(\rho_{1}^{+}\left(\hat{e}_{1}, \hat{z}_{1}\right)\right)+(1-\gamma) V\left(\rho_{1}^{++}\left(\hat{e}_{1}, \hat{z}_{1}\right)\right)\right]$. The rest of the proof contains 3 steps. Step 1 establishes than in equilibrium $e_{1}\left(\rho_{1}\right)<1$. Step 2 computes the probability with which the strategic agency must announce $r_{1}=-1$ if in equilibrium $e_{1}\left(\rho_{1}\right)=0$. Step 3 characterizes a parameter region in which $e_{1}\left(\rho_{1}\right)>0$.

Step 1: An equilibrium with $e_{1}\left(\rho_{1}\right)=1$ requires

$$
\delta(1-\gamma) V\left(\rho_{1}\right)-c \geq(1-\gamma) \Phi^{\dagger}\left(\rho_{1}, 1, z_{1}\right)
$$

for some $z_{1}$. Note that, for any $z_{1}$ and any $\rho_{1}: \Phi\left(\rho_{1}, 1, z_{1}\right)=1>V\left(\rho_{1}\right)$. Thus (65) is violated as $c>0$ and $\delta<1$. Hence in equilibrium $e\left(\rho_{1}\right)<1$.

Step 2: Define $\tilde{z}\left(\rho_{1}\right)$ implicitly by

$$
\Phi^{\dagger}\left(\rho_{1}, 0, \tilde{z}\left(\rho_{1}\right)\right)=\delta\left[(1-\gamma) V\left(\rho_{1}^{++}\left(0, \tilde{z}\left(\rho_{1}\right)\right)\right)-\gamma V\left(\rho_{1}^{+}\left(0, \tilde{z}\left(\rho_{1}\right)\right)\right)\right]
$$


which is equivalent to

$$
\frac{\rho_{1} \gamma}{1-\rho_{1}(1-\gamma)-\left(1-\rho_{1}\right) \tilde{z}}=\delta\left(\frac{\rho_{1} \gamma(1-\gamma)}{\rho_{1}+\left(1-\rho_{1}\right) \tilde{z}-(1-\gamma) \rho_{1}}-\frac{\gamma^{2} \rho_{1}}{1-\rho_{1}(1-\gamma)-\left(1-\rho_{1}\right) \tilde{z}}\right)
$$

Simplifying to solve for $\tilde{z}$ gives $\tilde{z}\left(\rho_{1}\right)=\frac{\delta(1-\gamma)}{1+\delta}+\frac{\rho_{1} \gamma}{1-\rho_{1}} \frac{\delta(1-2 \gamma)-1}{1+\delta}$. Note that $\tilde{z}\left(\rho_{1}\right)<1$ if and only if $\rho_{1}<\frac{1+\gamma \delta}{(1+\gamma \delta)-\left(\gamma(1-\delta)+2 \delta \gamma^{2}\right)}$ and this condition is satisfied for all $\rho_{1} \in(0,1)$; furthermore, $\tilde{z}\left(\rho_{1}\right)>0$ if and only if $\rho_{1}<\rho_{1}^{\dagger}(\delta)$ where $\rho_{1}^{\dagger}(\delta):=\frac{(1-\gamma) \delta}{(1-\gamma) \delta+\left(\gamma(1-\delta)+2 \gamma^{2} \delta\right)}$. Note that $\rho_{1}^{\dagger}(\delta) \in(0,1)$. By construction of $\tilde{z}\left(\rho_{1}\right)$, in equilibrium, $e_{1}\left(\rho_{1}\right)=0$ implies $z\left(\rho_{1}\right)=\tilde{z}\left(\rho_{1}\right)$ if $\rho_{1} \leq \rho_{1}^{\dagger}(\delta)$ and $z\left(\rho_{1}\right)=0$ if $\rho_{1}>\rho_{1}^{\dagger}(\delta)$.

Step 3: Define $\bar{\rho}^{\dagger}(\delta)=\frac{\delta(1-\gamma)-c}{\delta(1-\gamma)^{2}+(\gamma-c)(1-\gamma)}, \underline{\rho}^{\dagger}(\delta):=\frac{c(1+\delta \gamma)}{\delta^{2} \gamma^{2}-2 c \delta \gamma^{2}+c \delta \gamma+2 \delta \gamma^{2}-2 c \gamma+\gamma^{2}+c}$. Consider first $\rho_{1} \geq \rho_{1}^{\dagger}(\delta)$. Step 2 ensures that an equilibrium with $e_{1}\left(\rho_{1}\right)=0$ exists if and only if: $(1-$ $\gamma) \Phi^{\dagger}\left(\rho_{1}, 0,0\right) \geq(1-\gamma) \delta V(1)-c$. This condition is equivalent to: $\rho_{1} \leq \bar{\rho}^{\dagger}(\delta)$. We conclude that if $\rho_{1} \in\left[\rho_{1}^{\dagger}(\delta), \bar{\rho}^{\dagger}(\delta)\right)$ in equilibrium $e_{1} \in(0,1)$. Now consider a $\rho_{1} \leq \rho_{1}^{\dagger}(\delta)$. Step 2 ensures that an equilibrium with $e_{1}\left(\rho_{1}\right)=0$ exists if and only if $(1-\gamma) \Phi^{\dagger}\left(\rho_{1}, 0, \tilde{z}\left(\rho_{1}\right)\right)+c \geq$ $(1-\gamma) \delta V\left(\rho_{1}^{++}\left(0, \tilde{z}\left(\rho_{1}\right)\right)\right)$. This condition is equivalent to: $\rho_{1} \leq \rho^{c}(\delta)$. We conclude that if $\rho_{1}\left(\underline{\rho}^{c}(\delta), \rho_{1}^{\dagger}(\delta)\right)$ in equilibrium $e_{1} \in(0,1)$.

Finally, straightforward algebra shows that both $\bar{\rho}^{c}(\delta)>\rho^{\dagger}(\delta)$ and $\underline{\rho}^{c}(\delta)<\rho^{\dagger}(\delta)$ are equivalent to $c<\frac{\delta \gamma(1-\gamma)(1+\delta)}{1+\delta \gamma}$. This inequality is satisfied if $\delta \in(\underline{\delta}, 1)$ and violated if $\delta \in(0, \underline{\delta}]$, where $\underline{\delta}:=\frac{c \gamma+\gamma^{2}-\gamma+\sqrt{c^{2} \gamma^{2}+2 c \gamma^{3}+\gamma^{4}-6 c \gamma^{2}-2 \gamma^{3}+4 c \gamma+\gamma^{2}}}{2 \gamma(1-\gamma)}$. This concludes the proof.

Proof of Proposition 24: Note that $\Upsilon^{\dagger}$ is continuous, weakly increasing in each of its arguments, and $\Upsilon^{\dagger}(1, \cdot)=\Upsilon^{\dagger}(\cdot, 1)=\gamma$. Define $V(\rho):=\gamma \rho$. Let $\rho_{1}^{+}(e, z)$ (respectively $\rho_{1}^{++}(e, z)$ ) denote the value of $\rho_{1}^{+}$(respectively $\rho_{1}^{++}$) for $e_{1}\left(\rho_{1}\right)=e$ and $z_{1}\left(\rho_{1}\right)=z$. At $t=1$, the agency's expected intertemporal profit from shirking and announcing $r_{t}=1$ is equal to $\Upsilon^{\dagger}\left(\rho_{1}, \hat{e}_{1}\right)+\delta \gamma V\left(\rho_{1}^{+}\left(\hat{e}_{1}, \hat{z}_{1}\right)\right)$, the intertemporal profit from shirking and announcing $r_{t}=-1$ is equal to $\Upsilon^{\dagger}\left(\rho_{1}, \hat{e}_{1}\right)+\delta(1-\gamma) V\left(\rho_{1}^{++}\left(\hat{e}_{1}, \hat{z}_{1}\right)\right)$, and the intertemporal profit from acquiring information is equal to $\Upsilon^{\dagger}\left(\rho_{1}, \hat{e}_{1}\right)+\delta\left[\gamma V\left(\rho_{1}^{+}\left(\hat{e}_{1}, \hat{z}_{1}\right)\right)+(1-\gamma) V\left(\rho_{1}^{++}\left(\hat{e}_{1}, \hat{z}_{1}\right)\right)\right]-c$.

We now consider two cases. These cases together prove the proposition.

Case 1. Let $\gamma \in\left(0, \frac{1}{2}\right]$. In equilibrium, if $\delta<\frac{c}{\gamma^{2}}$ then $e_{1}\left(\rho_{1}\right)=0$ for all $\rho_{1} \in(0,1)$. If 
instead $\delta>\frac{c}{\gamma^{2}}$ then

$$
\left\{\begin{array}{l}
\text { if } \rho_{1} \in\left(\frac{c}{\delta \gamma^{2}}, 1\right) \quad \text { then } \quad e\left(\rho_{1}\right)=1, \\
\text { if } \rho_{1} \in\left(\frac{c}{\delta \gamma-c}, \frac{c}{\delta \gamma^{2}}\right) \quad \text { then } \quad e\left(\rho_{1}\right) \in(0,1), \\
\text { if } \quad \rho_{1} \in\left(0, \frac{c}{\delta \gamma-c}\right) \quad \text { then } \quad e\left(\rho_{1}\right)=0 .
\end{array}\right.
$$

Define $\tilde{z}\left(\rho_{1}\right)$ implicitly as follows:

$$
\gamma V\left(\rho_{1}^{+}\left(0, \tilde{z}\left(\rho_{1}\right)\right)\right)=(1-\gamma) V\left(\rho_{1}^{++}\left(0, \tilde{z}\left(\rho_{1}\right)\right)\right)
$$

Solving (66) we obtain $\tilde{z}\left(\rho_{1}\right)=1-\gamma+(1-2 \gamma) \frac{\rho_{1}}{1-\rho_{1}}$.

As $\gamma \in\left(0, \frac{1}{2}\right]$, then $\tilde{z}\left(\rho_{1}\right)>0$ for all $\rho_{1}$, while $\tilde{z}\left(\rho_{1}\right)<1$ if and only if $\rho_{1}<\frac{\gamma}{1-\gamma}$, where $\frac{\gamma}{1-\gamma} \in(0,1)$. By construction of $\tilde{z}\left(\rho_{1}\right)$, in equilibrium $e_{1}\left(\rho_{1}\right)=0$ implies $z\left(\rho_{1}\right)=\tilde{z}\left(\rho_{1}\right)$ if $\rho_{1}<\frac{\gamma}{1-\gamma}$, and $z\left(\rho_{1}\right)=1$ if $\rho_{1} \geq \frac{\gamma}{1-\gamma}$.

Fix first $\rho_{1} \geq \frac{\gamma}{1-\gamma}$. An equilibrium with $e_{1}\left(\rho_{1}\right)=0$ exists if and only if $\delta \gamma V\left(\rho_{1}^{+}(0,1)\right) \leq c$, which is equivalent to $\delta \leq \frac{c}{\gamma^{2}}$. Fix $\delta<\frac{c}{\gamma^{2}}$. As $V\left(\rho_{1}^{+}(0,1)\right)=V(1) \geq V\left(\rho_{2}\right)$ for all $\rho_{2} \in[0,1]$, then for and any $e_{1}$ and $z_{1}: \delta \gamma V\left(\rho_{1}^{+}\left(e_{1}, z_{1}\right)\right)<c$. Hence in any equilibrium $e_{1}\left(\rho_{1}\right)=0$ (and $\left.z_{1}\left(\rho_{1}\right)=1\right)$.

Fix now $\rho_{1}<\frac{\gamma}{1-\gamma}$. An equilibrium with $e_{1}\left(\rho_{1}\right)=0$ exists if and only if $\delta \gamma V\left(\rho_{1}^{+}\left(0, \tilde{z}\left(\rho_{1}\right)\right)\right) \leq$ $c$, which is equivalent to $\rho_{1} \leq \frac{c}{\delta \gamma-c}$. Fix $\rho_{1}<\frac{c}{\delta \gamma-c}$. Clearly in this region if in equilibrium with $z_{1}\left(\rho_{1}\right)=\tilde{z}\left(\rho_{1}\right)$ then $e_{1}\left(\rho_{1}\right)=0$. Suppose an equilibrium exists in which $z_{1}\left(\rho_{1}\right)>\tilde{z}\left(\rho_{1}\right)$; note that for any $\hat{e}_{1}: \delta(1-\gamma) V\left(\rho_{1}^{++}\left(\hat{e}_{1}, z_{1}\left(\rho_{1}\right)\right)\right) \leq \delta(1-\gamma) V\left(\rho_{1}^{++}\left(0, z_{1}\left(\rho_{1}\right)\right)\right)<$ $\delta(1-\gamma) V\left(\rho_{1}^{++}\left(0, \tilde{z}_{1}\left(\rho_{1}\right)\right)\right)=\delta \gamma V\left(\rho_{1}^{+}\left(0, \tilde{z}_{1}\left(\rho_{1}\right)\right)\right)<c$, hence in such an equilibrium $e_{1}\left(\rho_{1}\right)=0$. Suppose now that an equilibrium exists in which $z_{1}\left(\rho_{1}\right)<\tilde{z}\left(\rho_{1}\right)$; note that for any $\hat{e}_{1}$ : $\delta \gamma V\left(\rho_{1}^{+}\left(\hat{e}_{1}, z_{1}\left(\rho_{1}\right)\right)\right) \leq \delta \gamma V\left(\rho_{1}^{+}\left(0, z_{1}\left(\rho_{1}\right)\right)\right)<\delta \gamma V\left(\rho_{1}^{+}\left(0, \tilde{z}_{1}\left(\rho_{1}\right)\right)\right)<c$, hence in such an equilibrium $e_{1}\left(\rho_{1}\right)=0$. Hence in any equilibrium $e_{1}\left(\rho_{1}\right)=0\left(\right.$ and $\left.z_{1}\left(\rho_{1}\right)=\tilde{z}\left(\rho_{1}\right)\right)$.

Note that, for $\delta<\frac{c}{\gamma^{2}}, \frac{c}{\delta \gamma-c}>1 \geq \rho_{1}$ for all $\rho_{1}$. Hence if either $\delta<\frac{c}{\gamma^{2}}$ or $\delta>\frac{c}{\gamma^{2}}$ and $\rho_{1}<\frac{c}{\delta \gamma-c}$ then in equilibrium $e_{1}\left(\rho_{1}\right)=0$.

Consider now an equilibrium in which $e_{1}\left(\rho_{1}\right)=1$. In such equilibrium $\rho_{1}^{++}=\rho_{1}^{+}=\rho_{1}$. As $\gamma \leq \frac{1}{2}$, in such equilibrium $\gamma V\left(\rho_{1}^{+}\right)<(1-\gamma) V\left(\rho_{1}^{++}\right)$, hence $z_{1}\left(\rho_{1}\right)=1$. Thus an equilibrium with $e_{1}\left(\rho_{1}\right)=1$ exists if and only if $\delta \gamma V\left(\rho_{1}\right) \geq c$ that is, if and only if $\rho_{1} \geq \frac{c}{\delta \gamma^{2}}$. Let $\rho_{1}>\frac{c}{\delta \gamma^{2}}$. Note that for any $e_{1}$ and $z_{1}, \rho_{1}^{+}\left(e_{1}, z_{1}\right) \geq \rho_{1}$, hence $\delta \gamma V\left(\rho_{1}^{+}\right)>c$, i.e. there is no equilibrium in which the agency shirks and deflates with positive probability. Moreover, as $\gamma \leq \frac{1}{2}: \delta(1-\gamma) V\left(\rho_{1}^{+}\right) \geq \delta(1-\gamma) V\left(\rho_{1}\right) \geq \delta \gamma V\left(\rho_{1}\right)>c$, i.e. there is no equilibrium in which 
the agency shirks and inflates with positive probability. Thus for $\rho_{1}>\frac{c}{\delta \gamma^{2}}$ in equilibrium $e_{1}(\rho)=1$. Noting that an equilibrium exists for all parameter values concludes the proof of case 1 .

Case 2. Let $\gamma \in\left(\frac{1}{2}, 1\right)$. In equilibrium, if $\delta<\frac{c}{\gamma(1-\gamma)}$ then $e_{1}\left(\rho_{1}\right)=0$ for all $\rho_{1} \in(0,1)$. If instead $\delta>\frac{c}{\gamma(1-\gamma)}$ then

$$
\left\{\begin{array}{l}
\text { if } \quad \rho_{1} \in\left(\frac{c}{\delta \gamma(1-\gamma)}, 1\right) \quad \text { then } \quad e\left(\rho_{1}\right)=1, \\
\text { if } \quad \rho_{1} \in\left(\frac{c}{\delta \gamma-c}, \frac{c}{\delta \gamma(1-\gamma)}\right) \quad \text { then } \quad e\left(\rho_{1}\right) \in(0,1), \\
\text { if } \quad \rho_{1} \in\left(0, \frac{c}{\delta \gamma-c}\right) \quad \text { then } \quad e\left(\rho_{1}\right)=0 .
\end{array}\right.
$$

Consider $\tilde{z}\left(\rho_{1}\right)$, as defined by $(66)$. As $\gamma \in\left(\frac{1}{2}, 1\right)$, then $\tilde{z}\left(\rho_{1}\right)<1$ for all $\rho_{1}$, while $\tilde{z}\left(\rho_{1}\right)>0$ if and only if $\rho_{1}<\frac{1-\gamma}{\gamma}$, where $\frac{1-\gamma}{\gamma} \in(0,1)$. By construction of $\tilde{z}\left(\rho_{1}\right)$, in equilibrium $e_{1}\left(\rho_{1}\right)=0$ implies $z\left(\rho_{1}\right)=\tilde{z}\left(\rho_{1}\right)$ if $\rho_{1}<\frac{1-\gamma}{\gamma}$, and $z\left(\rho_{1}\right)=0$ if $\rho_{1} \geq \frac{1-\gamma}{\gamma}$.

So for $\rho_{1} \geq \frac{1-\gamma}{\gamma}$ an equilibrium with $e_{1}\left(\rho_{1}\right)=0$ exists if and only if $\delta(1-\gamma) V\left(\rho_{1}^{++}(0,0)\right) \leq c$, which is equivalent to $\delta \leq \frac{c}{\gamma(1-\gamma)}$. Consider $\delta<\frac{c}{\gamma(1-\gamma)}$. As $V\left(\rho_{1}^{++}(0,0)\right)=V(1) \geq V\left(\rho_{2}\right)$ for all $\rho_{2}$, then for any $e_{1}$ and $z_{1}: \delta(1-\gamma) V\left(\rho_{1}^{++}\left(e_{1}, z_{1}\right)\right)<c$. Thus in this region, in equilibrium, $e_{1}\left(\rho_{1}\right)=0$.

For $\rho_{1}<\frac{1-\gamma}{\gamma}$ instead, an equilibrium with $e_{1}\left(\rho_{1}\right)=0$ exists if and only if $\delta \gamma V\left(\rho_{1}^{+}\left(0, \tilde{z}\left(\rho_{1}\right)\right)\right) \leq$ $c$, which is equivalent to $\rho_{1} \leq \frac{c}{\delta \gamma-c}$. The proof that in equilibrium $e_{1}\left(\rho_{1}\right)=0$ for $\rho_{1}<\frac{c}{\delta \gamma-c}$ follows the same steps as the proof that $e_{1}\left(\rho_{1}\right)=0$ for $\gamma \in\left(0, \frac{1}{2}\right]$ and $\rho_{1}<\frac{c}{\delta \gamma-c}$ discussed above.

Consider now an equilibrium in which $e_{1}\left(\rho_{1}\right)=1$. As $\gamma>\frac{1}{2}$, in such equilibrium $z_{1}\left(\rho_{1}\right)=0$. Thus an equilibrium with $e_{1}\left(\rho_{1}\right)=1$ exists if and only if $\delta(1-\gamma) V\left(\rho_{1}\right) \geq c$ that is, if and only if $\rho_{1} \geq \frac{c}{\delta \gamma(1-\gamma)}$. Let $\rho_{1}>\frac{c}{\delta \gamma(1-\gamma)}$. Note that for any $e_{1}$ and $z_{1}, \rho_{1}^{++}\left(e_{1}, z_{1}\right) \geq \rho_{1}$, hence $\delta(1-\gamma) V\left(\rho_{1}^{++}\right)>c$. Moreover, as $\gamma>\frac{1}{2}: \delta \gamma V\left(\rho_{1}^{+}\right) \geq \delta \gamma V\left(\rho_{1}\right)>\delta(1-\gamma) V\left(\rho_{1}\right)>c$. Thus for $\rho_{1}>\frac{c}{\delta \gamma(1-\gamma)}$ in equilibrium $e_{1}(\rho)=1$. Noting that an equilibrium exists for all parameter values concludes the proof of case 2 .

Proof of Remark 1: As shown in the Proof of Proposition 23, $\delta>\underline{\delta}$ implies

$$
c<\frac{\delta \gamma(1-\gamma)(1+\delta)}{1+\delta \gamma}
$$


We prove the remark by showing that for $\delta=\frac{c}{\gamma x}$ condition (67) holds. We consider the cases $\gamma \leq \frac{1}{2}$ and $\gamma>\frac{1}{2}$ separately.

Let $\gamma \leq \frac{1}{2}$, hence $x=\gamma$. For $\delta=\frac{c}{\gamma^{2}}$, (67) reduces to:

$$
\begin{gathered}
c<\frac{\frac{c}{\gamma}(1-\gamma)\left(1+\frac{c}{\gamma^{2}}\right)}{1+\frac{c}{\gamma}} \quad \Leftrightarrow \\
(2 \gamma-1) \gamma^{2}<\left(1-\gamma-\gamma^{2}\right) c .
\end{gathered}
$$

This last inequality holds as $(2 \gamma-1) \gamma^{2}<0<\left(1-\gamma-\gamma^{2}\right) c$.

Let now $\gamma>\frac{1}{2}$, hence $x=1-\gamma$. For $\delta=\frac{c}{\gamma(1-\gamma)},(67)$ reduces to:

$$
c<\frac{\frac{c}{\gamma(1-\gamma)} \gamma(1-\gamma)\left(1+\frac{c}{\gamma(1-\gamma)}\right)}{1+\frac{c}{\gamma(1-\gamma)} \gamma}
$$

This condition is equivalent to $\gamma<1$, which clearly holds. 


\section{Online Appendix E}

In this appendix we check the robustness of our analysis by assuming that investors and firms never observe $q$. We show that, as in the baseline model:

- for some parameter values contingent fees improve expected social welfare relative to upfront fees;

- upfront fees improve expected social welfare relative to contingent fees as long as the cost of information acquisition, $c$, is sufficiently small.

The Model. The agency lives for two periods. The model differs from the one discussed in Section 3 only in that investors and firms never observe $q_{t}$ (and we set $\beta=1$ ).

Given $e:[0,1] \rightarrow[0,1]$, define

$$
\rho^{+}:=\left\{\begin{array}{ll}
0 & \text { if } \rho=0 \\
\frac{\rho}{\rho+(1-\rho) e(\rho)} & \text { if } \rho>0
\end{array} \quad ; \quad \rho^{-}:= \begin{cases}0 & \text { if } \rho=0 \\
\frac{\rho}{1+(1-\rho)(1-e)} & \text { if } \rho>0\end{cases}\right.
$$

The next definition is immediately adapted from the equilibrium concept of Section 2 .

Definition 5. An equilibrium with contingent fees comprises functions $e_{t}:[0,1] \rightarrow[0,1]$ specifying the probabilities $e_{t}\left(\rho_{t}\right)$ that the strategic agency acquires information in period $t$ given reputation $\rho_{t}$, for $t=1,2$, such that each period:

(i) the choice(s) implied by $e_{t}\left(\rho_{t}\right)$ maximize the agency's expected intertemporal profit given by (35), and

$$
\rho_{2}= \begin{cases}\rho_{1}^{+} & \text {if } r_{1}=-1 \\ \rho_{1}^{-} & \text {if } r_{1}=1\end{cases}
$$

(ii) firms and investors' beliefs satisfy $\hat{e}_{t}=e_{t}\left(\rho_{t}\right)$.

The definition of an equilibrium with upfront fees is obtained by replacing (35) with (36).

The Equilibria. We next characterize the equilibria with, respectively, contingent fees and upfront fees. Proofs of the propositions are relegated to the end of this appendix. 
Proposition 25. With contingent fees, in equilibrium $e_{1}\left(\rho_{1}\right)>0$ if and only if $\delta>2 c$ and $\rho_{1}<\rho_{c}(\delta)$, where $\rho_{c}(\delta)$ is defined implicitly by

$$
\delta=\left(\frac{4-3 \rho_{c}}{2\left(1-\rho_{c}\right)}\right)\left(\frac{\rho_{c}}{2-\rho_{c}}+c\right) .
$$

Moreover $\lim _{c \rightarrow 0} e_{1}\left(\rho_{1}\right)<1$ for any $\rho_{1}$ and $\delta$.

Proposition 26. With upfront fees, in equilibrium $e_{1}\left(\rho_{1}\right)>0$ if and only if $\delta>4 c$ and $\rho_{1}<\rho_{u}(\delta):=\frac{4 c-\delta}{2-\delta}$. Moreover $\lim _{c \rightarrow 0} e_{1}\left(\rho_{1}\right)=1$ for any $\rho_{1}$ and $\delta$.

Welfare Comparison. When fees are contingent, for $\delta \in(2 c, 4 c)$ the strategic agency acquires information at $t=1$ with positive probability provided its reputation is not too high (Proposition 25). By contrast, for $\delta \in(2 c, 4 c)$ the strategic agency shirks with probability 1 when fees are upfront (Proposition 26). For $\delta \in(2 c, 4 c)$ and sufficiently low reputation expected social welfare is therefore higher under contingent fees than under upfront fees. Moreover for any $\delta$ and $\rho_{1}, \lim _{c \rightarrow 0} e_{1}\left(\rho_{1}\right)=1$ only in the case of upfront fees. Thus upfront fees increase expected social welfare if the cost of information acquisition is sufficiently low.

Proof of Proposition 25: Define $V(\rho):=\frac{\rho}{2-\rho}$. At $t=1$, the agency's expected intertemporal profit from shirking and announcing $r_{t}=1$ is equal to $\Phi\left(\rho_{1}, \hat{e}_{1}\right)+\delta V\left(\rho_{1}^{-}\right)$. The intertemporal profit from acquiring information is equal to $\frac{1}{2} \Phi\left(\rho_{1}, \hat{e}_{1}\right)-c+\frac{\delta}{2}\left[V\left(\rho_{1}^{+}\right)+V\left(\rho_{1}^{-}\right)\right]$.

Fix $\rho_{1} \in(0,1)$. An equilibrium in which $e_{1}\left(\rho_{1}\right)=1$ exists if and only if

$$
\Phi\left(\rho_{1}, 1\right)+\delta V\left(\rho_{1}\right) \leq \frac{1}{2} \Phi\left(\rho_{1}, 1\right)-c+\delta V\left(\rho_{1}\right)
$$

This condition is always violated, thus in equilibrium $e_{1}\left(\rho_{1}\right)<1$. An equilibrium in which $e_{1}\left(\rho_{1}\right)=0$ in turn exists if and only if

$$
\Phi\left(\rho_{1}, 0\right)+\delta V\left(\frac{\rho_{1}}{2-\rho_{1}}\right) \geq \frac{1}{2} \Phi\left(\rho_{1}, 0\right)+\frac{\delta}{2}\left(V(1)+V\left(\frac{\rho_{1}}{2-\rho_{1}}\right)\right)-c .
$$

Substituting for $\Phi(\cdot)$ and $V(\cdot)$ and simplifying yields

$$
\delta \leq \tilde{\delta}\left(\rho_{1}\right):=\left(\frac{4-3 \rho_{1}}{2\left(1-\rho_{1}\right)}\right)\left(\frac{\rho_{1}}{2-\rho_{1}}+c\right)
$$


Notice that $\tilde{\delta}\left(\rho_{1}\right)$ is continuous and increasing in $\rho_{1}$ for $\rho_{1} \in(0,1)$ as it is the product of 2 terms, each of which is continuous and increasing in $\rho_{1}$ for $\rho_{1} \in(0,1)$. Hence $\delta<\tilde{\delta}\left(\rho_{1}\right)$ is equivalent to $\rho_{1}>\rho_{c}(\delta)$; moreover as $\tilde{\delta}(0)=2 c$, then $e_{1}\left(\rho_{1}\right)>0$ only if $\delta>2 c$. The proof that $\delta>\tilde{\delta}\left(\rho_{1}\right)$ is necessary and sufficient for an equilibrium in which $e_{1} \in(0,1)$ follows standard arguments.

Pick a pair $\delta$ and $\rho_{1}$. For sufficiently small $c, \delta>2 c$, thus in equilibrium $e_{1}\left(\rho_{1}\right) \in(0,1)$. Note also that for $c=0$ and $e\left(\rho_{1}\right)=1$ (68) is violated. Thus, by continuity of $V$ and $\Phi$ in equilibrium $\lim _{c \rightarrow 0} e_{1}\left(\rho_{1}\right)<1$.

Proof of Proposition 26: Define $V(\rho):=\frac{\rho}{2}$. At $t=1$, the agency's expected intertemporal profit from shirking and announcing $r_{t}=1$ is equal to $\Upsilon\left(\rho_{1}, \hat{e}_{1}\right)+\delta V\left(\rho_{1}^{-}\right)$. The intertemporal profit from acquiring information is equal to $\Upsilon\left(\rho_{1}, \hat{e}_{1}\right)-c+\frac{\delta}{2}\left[V\left(\rho_{1}^{+}\right)+V\left(\rho_{1}^{-}\right)\right]$. Fix $\rho_{1} \in(0,1)$. An equilibrium in which $e_{1}\left(\rho_{1}\right)=1$ exists if and only if

$$
\delta V\left(\rho_{1}\right) \leq \delta V\left(\rho_{1}\right)-c
$$

This condition is always violated for $c>0$. An equilibrium in which $e_{1}\left(\rho_{1}\right)=0$ exists if and only if

$$
\frac{\delta}{2} V\left(\frac{\rho_{1}}{2-\rho_{1}}\right) \geq \frac{\delta}{2} V(1)-c .
$$

Substituting for $V(\cdot)$ and simplifying yields

$$
\delta \leq \bar{\delta}\left(\rho_{1}\right):=\frac{2 c\left(2-\rho_{1}\right)}{1-\rho_{1}}
$$

As $\bar{\delta}\left(\rho_{1}\right)$ is continuous and increasing in $\rho_{1}$, then $\delta<\bar{\delta}\left(\rho_{1}\right)$ is equivalent to $\rho_{1}>\rho_{u}(\delta)$; moreover, as $\bar{\delta}(0)=4 c$, then $e_{1}\left(\rho_{1}\right)>0$ only if $\delta>4 c$. The proof that $\delta>\bar{\delta}\left(\rho_{1}\right)$ is necessary and sufficient for an equilibrium in which $e_{1} \in(0,1)$ follows standard arguments.

Next, fix $\delta$ and $\rho_{1}$. Note also that, for $c=0, e\left(\rho_{1}\right)=1$ satisfies (69). Continuity of $\Upsilon$ and $V$ thus ensure that in equilibrium $\lim _{c \rightarrow 0} e_{1}\left(\rho_{1}\right)=1$. 


\section{Online Appendix F}

In this appendix we present and analyze the model with observable information acquisition. The model and the main result of this appendix (Proposition 27) are presented in Subsection F.1. The analysis of an auxiliary game needed in the proof of Proposition 27 is carried out in Subsection F.2. Subsection F.3 contains the proof of Proposition 27.

\section{F.1 The model}

Fees. Each period, firms and investors form beliefs regarding the probability that the strategic agency will truthfully report what it observes. Let $\hat{a}_{t}$ denote the beginning-of-period- $t$ belief that, conditional on acquiring information and observing $q_{t}=-1$, the strategic agency truthfully assigns the rating $r_{t}=-1$. We maintain the notation $\hat{e}_{t}$ for the beginning-of-period- $t$ belief that the strategic agency will acquire information in period $t$, and set $\beta=1$ to reduce notation. So the contingent fee is

$$
\phi_{t}\left(r_{t}\right)= \begin{cases}\mathbb{E}\left[q_{t} \mid r_{t}=1, \rho_{t}, \hat{e}_{t}, \hat{a}_{t}\right] & \text { if } r_{t}=1 \\ 0 & \text { if } r_{t}=-1\end{cases}
$$

while the upfront fee is $\phi_{t}(1)=\phi_{t}(-1)=\mathbb{P}\left(r_{t}=1 \mid \rho_{t}, \hat{e}_{t}, \hat{a}_{t}\right) \mathbb{E}\left[q_{t} \mid r_{t}=1, \rho_{t}, \hat{e}_{t}, \hat{a}_{t}\right]$.

Timing. The timing within period $t$ is as follows. The agency first decides whether or not to acquire information. In case the agency shirks the game moves on to the next period. This captures the idea that a regulatory authority prevents the agency from rating firm $t$ in case the agency is caught shirking. In case it chose to acquire information and observed $q_{t}=1$ the agency publicly announces the rating $r_{t}=1$. If it observed $q_{t}=-1$ the agency chooses whether to truthfully assign $r_{t}=-1$, or inflate the rating and assign $r_{t}=1$. The agency then receives $\phi_{t}\left(r_{t}\right)$, all players observe $q_{t}$ and the game moves on to the next period.

Strategies and Payoffs. A stationary strategy for the agency now comprises a pair $(e(\cdot), a(\cdot))$, where $e:[0,1] \rightarrow[0,1]$ and $a:[0,1] \rightarrow[0,1]$, specifying respectively the probability of acquiring information and the probability of truthfully assigning the rating $r_{t}=-1$ conditional on observing $q_{t}=-1$, both expressed as a function of the agency's reputation $\rho_{t}$. The payoffs are as in Section 2. The next definition adapts the equilibrium concept of that section. 
Definition 6. An equilibrium with observable information acquisition comprises a pair $(e(\cdot), a(\cdot))$ such that:

(i) in period $t$, the strategic agency acquires information with probability $e\left(\rho_{t}\right)$ and, conditional on observing $q_{t}=-1$, truthfully assigns the rating $r_{t}=-1$ with probability $a\left(\rho_{t}\right)$;

(ii) the strategy $(e(\cdot), a(\cdot))$ maximizes the agency's expected intertemporal profit given $\rho_{t+1}=$ $\Psi\left(\rho_{t}, r_{t}, q_{t}\right)$, where ${ }^{36}$

$$
\Psi\left(\rho_{t}, r_{t}, q_{t}\right):= \begin{cases}\frac{\rho_{t}}{\rho_{t}+\left(1-\rho_{t}\right) \hat{e}_{t}} & \text { if } q_{t}=1=r_{t} \text { and } \rho_{t}>0 ; \\ \frac{\rho_{t}}{\rho_{t}+\left(1-\rho_{t}\right) \hat{e}_{t} \hat{a}_{t}} & \text { if } q_{t}=-1=r_{t} \text { and } \rho_{t}>0 ; \\ 0 & \text { if } r_{t}=\emptyset, \text { or } q_{t}=-1=-r_{t}, \text { or } \rho_{t}=0 ;\end{cases}
$$

(iii) firms and investors' beliefs satisfy $\hat{e}_{t}=e\left(\rho_{t}\right)$ and $\hat{a}_{t}=a\left(\rho_{t}\right)$.

In equilibrium investors correctly infer the probabilities with which the strategic agency chooses to acquire information and to truthfully assign $r_{t}=-1$ when observing $q_{t}=-1$, and these choices are optimal for the agency. Firms and investors' beliefs are updated using Bayes' rule whenever possible. The agency loses its reputation whenever it is caught shirking. If it acquires information and $q_{t}=1$ then reputation is updated based on the belief $\hat{e}_{t}=e\left(\rho_{t}\right)$ alone, that is, reputation jumps up to

$$
\rho_{t}^{+}:=\frac{\rho_{t}}{\rho_{t}+\left(1-\rho_{t}\right) e\left(\rho_{t}\right)}
$$

By contrast, two cases arise if the agency acquires information and $q_{t}=-1: r_{t}=1$ reveals that the agency inflated the rating (and thus, that the agency is strategic), and $r_{t}=-1$ that the agency truthfully reported what it observed. In the latter case reputation is updated based both on the belief $\hat{e}_{t}=e\left(\rho_{t}\right)$ and on the belief $\hat{a}_{t}=a\left(\rho_{t}\right)$, that is, reputation jumps up to $^{37}$

$$
\rho_{t}^{++}:=\frac{\rho_{t}}{\rho_{t}+\left(1-\rho_{t}\right) e\left(\rho_{t}\right) a\left(\rho_{t}\right)} .
$$

We proceed to characterize the equilibrium behavior of the strategic agency. If the agency shirks, the agency is revealed to be strategic and the game moves on to the next period. So in

\footnotetext{
${ }^{36}$ We let $r_{t}=\emptyset$ denote the case in which the agency shirks in period $t$.

${ }^{37}$ As usual zero-probability events are dealt with by assuring that $\rho_{t}=0$ is an absorbing state of the Markov process, and by ascribing any misreporting to the strategic agency.
} 
order to obtain a positive payoff the agency is now forced to acquire information. Conditional on acquiring information, the agency can inflate the rating in case $q_{t}=-1$ or truthfully report what it observes. Inflating the rating guarantees the fee $\phi_{t}(1)$. The downside is that the agency could lose its reputation: either $q_{t}=1$ in which case $\rho_{t+1}=\rho_{t}^{+}$, or $q_{t}=-1$ in which case $\rho_{t+1}=0$. By contrast, truthfully reporting what the agency observes lowers the probability of receiving $\phi_{t}(1)$ to just $\frac{1}{2}$, but could induce a reputation boost: either $q_{t}=1$ in which case $\rho_{t+1}=\rho_{t}^{+}$, or $q_{t}=-1$ in which case $\rho_{t+1}=\rho_{t}^{++}$. By virtue of Bellman's Principle of Optimality an equilibrium with value function $V(\cdot)$ therefore satisfies the Bellman equation

$$
V(\rho)=\max \left\{\phi(\rho)-c+\delta\left(\frac{1}{2} V\left(\rho^{+}\right)+\frac{1}{2} V(0)\right), \frac{\phi(\rho)}{2}-c+\delta\left(\frac{1}{2} V\left(\rho^{+}\right)+\frac{1}{2} V\left(\rho^{++}\right)\right)\right\} .
$$

We show later that $V(0)=0$. Hence, the strategic agency is either indifferent between inflating the rating and truthful reporting, or $\frac{\phi(\rho)}{2}>\frac{\delta}{2} V\left(\rho^{++}\right)$in which case inflating the rating is uniquely optimal, or, lastly, $\frac{\phi(\rho)}{2}<\frac{\delta}{2} V\left(\rho^{++}\right)$in which case truthful reporting is uniquely optimal.

The following proposition is the main result of this appendix.

Proposition 27. With observable information acquisition:

1. if $\delta<\frac{2}{3-2 c}$ then upfront fees improve expected social welfare relative to contingent fees;

2. if $\delta>\frac{2}{3-2 c}$ then expected social welfare is the same whether fees are upfront or contingent.

\section{F.2 An Auxiliary Game}

We analyze in this subsection the auxiliary game in which, by assumption, the strategic agency (i) shirks if $\rho_{t}=0$, and (ii) acquires information if $\rho_{t}>0$. Hence, $e(0)=0$ and $e(\rho)=1$ for all $\rho>0$, and in this setting a stationary strategy for the agency is simply a mapping $a:(0,1] \rightarrow[0,1]$ specifying the probability of truthfully assigning the rating $r_{t}=-1$ when observing $q_{t}=-1$, as a function of the agency's reputation in period $t$. Our objective is to prove the following result:

Proposition 28. Let $\delta<\frac{2}{3-2 c}$. There exists a unique equilibrium of the auxiliary game. Its value function, $\tilde{V}(\cdot)$, is strictly increasing and continuous over $(0,1]$, and $\tilde{V}(1)>0$.

We start with two simple lemmas. Define, $\Xi(\cdot, \cdot):(0,1] \times[0,1] \times[0,1] \rightarrow \mathbb{R}$ such that

$$
\Xi(\rho, e, a):=\frac{\rho+(1-\rho) e a}{\rho+(1-\rho) e(2-a)} .
$$


Note that $\mathbb{E}\left[q_{t} \mid r_{t}=1, \rho_{t}, \hat{e}_{t}, \hat{a}_{t}\right]=\Xi\left(\rho_{t}, \hat{e}_{t}, \hat{a}_{t}\right)$ for all $\rho_{t}>0$, and that $\Xi(\cdot, \cdot, \cdot)$ is continuous, weakly increasing in $\rho$ and in $a$, weakly decreasing in $e, \Xi(\rho, e, a)>0$, and $\Xi(1, e, a)=$ $\Xi(\rho, e, 1)=\Xi(\rho, 0, a)=1$.

Given a function $a:[0,1] \rightarrow[0,1]$, define

$$
\rho^{\dagger}:= \begin{cases}0 & \text { if } \rho=0 \\ \frac{\rho}{\rho+(1-\rho) a(\rho)} & \text { if } \rho>0 .\end{cases}
$$

Lemma 13. In any equilibrium of the auxiliary game with value function $\tilde{V}(\cdot)$, for all $\rho>0$,

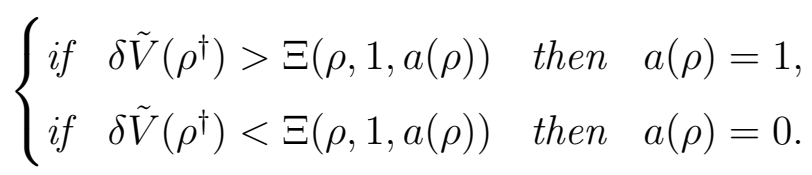

Proof: By virtue of Bellman's Principle of Optimality,

$$
\tilde{V}(\rho)=\max \left\{\Xi(\rho, 1, a(\rho))-c+\frac{\delta}{2} \tilde{V}(\rho), \frac{1}{2} \Xi(\rho, 1, a(\rho))-c+\delta\left(\frac{1}{2} \tilde{V}(\rho)+\frac{1}{2} \tilde{V}\left(\rho^{\dagger}\right)\right)\right\}
$$

for all $\rho>0$, and the choice implied by $a(\rho)$ maximizes the right-hand side of $(71)$.

Lemma 14. In any equilibrium of the auxiliary game,

$$
\left\{\begin{array}{l}
\text { if } a(\rho)=1 \text { then } \tilde{V}(\rho)=\frac{\frac{1}{2} \Xi(\rho, 1,1)-c}{1-\delta}=\max \left\{\frac{\frac{1}{2} \Xi(\rho, 1,1)-c}{1-\delta}, \frac{\Xi(\rho, 1,1)-c}{1-\frac{\delta}{2}}\right\}, \\
\text { if } a(\rho)<1 \text { then } \tilde{V}(\rho)=\frac{\Xi(\rho, 1, a(\rho))-c}{1-\frac{\delta}{2}} .
\end{array}\right.
$$

Furthermore, $\tilde{V}(1)=\max \left\{\frac{\frac{1}{2} \Xi(1,1,1)-c}{1-\delta}, \frac{\Xi(1,1,1)-c}{1-\frac{\delta}{2}}\right\} \geq \tilde{V}(\rho)$, for all $\rho \in[0,1]$.

Proof: The lemma follows from Bellman's Principle of Optimality together with the observations that (a) $a(\rho)=1$ implies $\rho^{\dagger}=\rho$, (b) $\rho=1$ implies $\rho^{\dagger}=1$, (c) $\Xi(1,1, a)=1$ for all $a \in[0,1]$, and $(\mathrm{d}) \Xi(\cdot, 1, \cdot)$ is weakly increasing in both variables.

Proof of Proposition 28: Define $\bar{\rho}$ and $\bar{a}$ implicitly by

$$
\Xi(\bar{\rho}, 1,0)=\delta\left(\frac{\Xi(1,1,1)-c}{1-\frac{\delta}{2}}\right),
$$


and

$$
\Xi(0,1, \bar{a})=\delta\left(\frac{\Xi(1,1,1)-c}{1-\frac{\delta}{2}}\right),
$$

respectively. As $\delta<\frac{2}{3-2 c} \Leftrightarrow \Xi(1,1,1)>\delta\left(\frac{\Xi(1,1,1)-c}{1-\frac{\delta}{2}}\right)$, we have $\bar{\rho}<1$ and $\bar{a}<1$. Noting that (since $c<\frac{\beta}{2}=\frac{1}{2}$ ) the right-hand side in (72) and (73) is strictly positive whereas $\Xi(0,1,0)=0$ yields $\bar{\rho}>0$ and $\bar{a}>0$. Thus $\bar{\rho} \in(0,1)$ and $\bar{a} \in(0,1)$.

We now show that there is a unique equilibrium of the auxiliary game, and that the equilibrium satisfies

$$
\begin{cases}a(\rho)=0 & \text { if } \rho \geq \bar{\rho} \\ a(\rho) \in(0, \bar{a}] & \text { if } \rho \in(0, \bar{\rho}) .\end{cases}
$$

We will first proceed by induction to show that there can exist at most one equilibrium. We will then argue that the inductive procedure yields an equilibrium.

As a preliminary step observe that, as $\delta<\frac{2}{3-2 c}$, then $\max \left\{\frac{\frac{1}{2} \Xi(1,1,1)-c}{1-\delta}, \frac{\Xi(1,1,1)-c}{1-\frac{\delta}{2}}\right\}=$ $\frac{\Xi(1,1,1)-c}{1-\frac{\delta}{2}}$. Hence, by Lemma 14

$$
\tilde{V}(\rho)=\frac{\Xi(\rho, 1, a(\rho))-c}{1-\frac{\delta}{2}},
$$

for all $\rho>0$.

The inductive procedure starts as follows. Combining (72) and (75) any equilibrium must be such that, for all $\rho>\bar{\rho}$ :

$$
\Xi(\rho, 1,0)>\delta \tilde{V}\left(\rho^{\dagger}\right)
$$

Thus, by Lemma 13, if an equilibrium exists it must satisfy $a(\rho)=0$ for all $\rho>\bar{\rho}$. A similar argument shows that in fact the same must be true for $\rho=\bar{\rho}$.

By contrast, consider $\rho \in(0, \bar{\rho})$. The combination of $(72),(75)$, and Lemma 13 shows that $a(\rho)=0$ is impossible in equilibrium. Similarly, the combination of (73), (75) and Lemma 13 shows that $a(\rho)>\bar{a}$ is impossible in equilibrium. Thus, any equilibrium must satisfy (74). By Lemma 13 this in turn implies that the indifference condition

$$
\delta \tilde{V}\left(\rho^{\dagger}\right)=\Xi(\rho, 1, a(\rho))
$$

must hold for all $\rho \in(0, \bar{\rho})$. 
Next define $\rho_{1}$ such that

$$
\bar{\rho}=\frac{\rho_{1}}{\rho_{1}+\left(1-\rho_{1}\right) \bar{a}} .
$$

Thus $\rho_{1}<\bar{\rho}$. By construction of $\rho_{1}$ and property (74), in any equilibrium: $\rho^{\dagger} \geq \bar{\rho}$ for all $\rho \in\left[\rho_{1}, \bar{\rho}\right)$. (76), (74) and (75) now pin down a unique candidate equilibrium $a(\rho)$ for each $\rho \in\left[\rho_{1}, \bar{\rho}\right.$ ) (which moreover is continuous in $\rho$ ). Repeating the step above with $\rho_{1}$ instead of $\bar{\rho}$ yields $\rho_{2}<\rho_{1}$ and a unique candidate equilibrium $a(\rho)$ for each $\rho \in\left[\rho_{2}, \rho_{1}\right)$, and so on. This defines a sequence $\left\{\rho_{n}\right\}$ where, for all $n, \rho_{n}=\frac{\rho_{n+1}}{\rho_{n+1}+\left(1-\rho_{n+1}\right) \bar{a}}$. As $\bar{a}<1, \rho_{n} \rightarrow 0$. This inductive procedure therefore pins down a unique candidate equilibrium, whose value function is continuous over $(0,1]$ (see $(75))$. That this candidate equilibrium is in fact an equilibrium is a consequence of the one-shot deviation principle.

It remains only to show that the value function $\tilde{V}$ of the unique equilibrium is strictly increasing over the interval $(0,1]$. We proceed by induction. $\tilde{V}$ is trivially increasing over $[\bar{\rho}, 1]$. Next, suppose that we can find $\rho_{a}$ and $\rho_{b}$ with $\bar{\rho}>\rho_{b}>\rho_{a} \geq \rho_{1}$ and $\Xi\left(\rho_{a}, 1, a\left(\rho_{a}\right)\right) \geq$ $\Xi\left(\rho_{b}, 1, a\left(\rho_{b}\right)\right)$. Then we must also have $a\left(\rho_{a}\right)>a\left(\rho_{b}\right)$, which in turn implies $\rho_{b}^{\dagger}>\rho_{a}^{\dagger} \geq \bar{\rho}$ and $\tilde{V}\left(\rho_{b}^{\dagger}\right)>\tilde{V}\left(\rho_{a}^{\dagger}\right)$. The latter inequality contradicts Lemma 13. Hence, $\Xi\left(\rho_{a}, 1, a\left(\rho_{a}\right)\right)<$ $\Xi\left(\rho_{b}, 1, a\left(\rho_{b}\right)\right)$. (75) thus yields $\tilde{V}\left(\rho_{a}\right)<\tilde{V}\left(\rho_{b}\right)$ and establishes that $\tilde{V}$ is increasing over $\left[\rho_{1}, 1\right]$. Repeating the step above with $\rho_{1}$ instead of $\bar{\rho}$, and so on, establishes that $\tilde{V}$ is increasing over $(0,1]$.

\section{F.3 Proof of Proposition 27}

Define $\Xi(\cdot, \cdot, \cdot):(0,1] \times[0,1] \times[0,1] \times \rightarrow \mathbb{R}$ such that

$$
\Xi(\rho, e, a):=\frac{\rho+(1-\rho) e a}{\rho+(1-\rho) e(2-a)} .
$$

Note that $\mathbb{E}\left[q_{t} \mid r_{t}=1, \rho_{t}, \hat{e}_{t}, \hat{a}_{t}\right]=\Xi\left(\rho_{t}, \hat{e}_{t}, \hat{a}_{t}\right)$ for all $\rho_{t}>0$, and that $\Xi(\cdot, \cdot, \cdot)$ is continuous, weakly increasing in $\rho$ and in $a$, weakly decreasing in $e, \Xi(\rho, e, a)>0$, and $\Xi(1, e, a)=$ $\Xi(\rho, e, 1)=\Xi(\rho, 0, a)=1$.

Given two functions $e:[0,1] \rightarrow[0,1]$ and $a:[0,1] \rightarrow[0,1]$, define

$$
\rho^{+}:= \begin{cases}0 & \text { if } \rho=0, \\ \frac{\rho}{\rho+(1-\rho) e(\rho)} & \text { if } \rho>0,\end{cases}
$$


and

$$
\rho^{++}:= \begin{cases}0 & \text { if } \rho=0 \\ \frac{\rho}{\rho+(1-\rho) e(\rho) a(\rho)} & \text { if } \rho>0 .\end{cases}
$$

Lemma 15. In any equilibrium, with $V(\cdot)$ denoting the value function of the equilibrium:

1. $e(0)=a(0)=V(0)=0$,

2. $e(\rho)>0$ for all $\rho>0$,

3. for all $\rho>0$ :

$$
\begin{aligned}
& V(\rho)= \\
& \max \left\{\Xi(\rho, e(\rho), a(\rho))-c+\frac{\delta}{2} V\left(\rho^{+}\right), \frac{1}{2} \Xi(\rho, e(\rho), a(\rho))-c+\delta\left(\frac{1}{2} V\left(\rho^{+}\right)+\frac{1}{2} V\left(\rho^{++}\right)\right)\right\} .
\end{aligned}
$$

Proof: Consider an arbitrary equilibrium; by virtue of Bellman's Principle of Optimality, since $\rho=0$ is an absorbing state, $a(0)>0$ implies $0 \geq \frac{1}{2} \mathbb{E}\left[q_{t} \mid r_{t}=1,0, e(0), a(0)\right]$. However, if $a(0)>0$ then $\mathbb{E}\left[q_{t} \mid r_{t}=1,0, e(0), a(0)\right]=\frac{a(0)}{2-a(0)}>0$. Thus, by contradiction, $a(0)=0$ and the fee of the agency with reputation $\rho_{t}=0$ is 0 . As $c>0$, for $\rho_{t}=0$ the agency's expected intertemporal profit from acquiring information in period $t$ is strictly negative. This implies $e(0)=0$ and $V(0)=0$.

Next observe that, whichever $e$ and $a$,

$$
V(1) \geq \frac{\frac{1}{2} \Xi(1, e, a)-c}{1-\delta}=\frac{\frac{1}{2}-c}{1-\delta}>0 .
$$

Thus $e(1)=1$, since each period the agency's payoff from shirking is 0 . Suppose now that $e(\hat{\rho})=0$ for some $\hat{\rho}>0$, and $\rho_{t}=\hat{\rho}$. Then by acquiring information in period $t$ the agency would (i) command in period $t$ the fee $\Xi(\hat{\rho}, 0, a(\hat{\rho}))=1$ and (ii) guarantee itself $\rho_{t+1}=1$. In other words, the agency's expected intertemporal profit from acquiring information in period $t$ equals $V(1)$. But then acquiring information strictly dominates shirking, contradicting the initial assumption that $e(\hat{\rho})=0$. This shows that $e(\rho)>0$ for all $\rho>0$. We can thus compute the value function of the equilibrium by conditioning on the strategic agency acquiring information, yielding the Bellman equation (77). The first expression on the righthand side is the expected intertemporal profit conditional on acquiring information and lying 
about $q_{t}$ in case $q_{t}=-1$. The second expression on the right-hand side is the expected intertemporal profit conditional on acquiring information and truthfully assigning $r_{t}=-1$ in case $q_{t}=-1$.

Proposition 29. Let $\delta>\frac{2}{3-2 c}$. There exists a unique equilibrium. In equilibrium:

1. $e(0)=a(0)=0$,

2. $e(\rho)=a(\rho)=1$, for all $\rho>0$.

Proof: Let $\hat{V}$ denote the value function corresponding to the strategy described in the statement of the proposition. Thus $\hat{V}(0)=0$ and $\hat{V}(\rho)=\frac{\frac{1}{2}-c}{1-\delta}>0$ for all, $\rho>0$. By virtue of the one-shot deviation principle if no single deviation is profitable then the strategy considered is an equilibrium. It is easy to see that there is no profitable deviation if $\rho_{t}=0$. It is also clear that if $\rho_{t}>0$ then shirking is not a profitable deviation. So we only have to check that if $\rho_{t}>0$ then lying about $q_{t}$ when $q_{t}=-1$ is not profitable either. That is, we have to check that $\Xi(\rho, 1,1) \leq \delta\left(\frac{\frac{1}{2}-c}{1-\delta}\right)$, which is equivalent to $\delta \geq \frac{2}{3-2 c}$.

We proceed to show that the strategy described in the statement of the proposition is the unique equilibrium. If $a(\rho)=1$ for all $\rho>0$ then $V(\rho) \geq \frac{\frac{1}{2}-c}{1-\delta}>0$ for all $\rho>0$. Therefore $a(\rho)=1$ for all $\rho>0$ implies $e(\rho)=1$ for all $\rho>0$. Next, suppose that an equilibrium exists such that $a(\hat{\rho})<1$ for some $\hat{\rho} \in(0,1)$. By virtue of $(77)$,

$$
\Xi(\hat{\rho}, e(\hat{\rho}), a(\hat{\rho})) \geq \delta V\left(\hat{\rho}^{++}\right)
$$

If $a\left(\hat{\rho}^{++}\right)$were equal to 1 we would then have (recall, from Lemma $15, e(\hat{\rho})>0$ ),

$$
1>\Xi(\hat{\rho}, e(\hat{\rho}), a(\hat{\rho})) \geq \delta\left(\frac{\frac{1}{2} \Xi\left(\hat{\rho}^{++}, e\left(\hat{\rho}^{++}\right), 1\right)-c}{1-\delta}\right),
$$

that is, $\delta<\frac{2}{3-2 c}$. Hence by contradiction $a\left(\hat{\rho}^{++}\right)<1$. We can thus repeat the steps above with $\hat{\rho}^{++}$instead of $\hat{\rho}$, and so on. This process determines a sequence $\left\{\rho_{n}\right\}$ such that, for all $n$ :

(i) $a\left(\rho_{n}\right)<1$,

(ii) $\rho_{n+1}=\frac{\rho_{n}}{\rho_{n}+\left(1-\rho_{n}\right) e\left(\rho_{n}\right) a\left(\rho_{n}\right)}>\rho_{n}$, 
(iii) $\Xi\left(\rho_{n}, e\left(\rho_{n}\right), a\left(\rho_{n}\right)\right) \geq \delta V\left(\rho_{n+1}\right) \geq \delta\left(\frac{\Xi\left(\rho_{n+1}, e\left(\rho_{n+1}\right), a\left(\rho_{n+1}\right)\right)-c}{1-\frac{\delta}{2}}\right)$.

By (i)-(ii), either $a\left(\rho_{n}\right) \rightarrow 1$ or $\rho_{n} \rightarrow 1$. Hence, taking limits in (iii) yields (using continuity of $\Xi(\cdot, \cdot, \cdot)$ and the fact that $\Xi(1, e, a)=\Phi(\rho, e, 1)=1$ for all $\rho, e$ and $a)$ :

$$
\Xi(1,1,1) \geq \delta\left(\frac{\Xi(1,1,1)-c}{1-\frac{\delta}{2}}\right)
$$

This simplifies to $\delta \leq \frac{2}{3-2 c}$.

Lemma 16. Let $\delta<\frac{2}{3-2 c}$. There exists $\bar{\rho} \in(0,1)$ and $\bar{a} \in(0,1)$ such that, in any equilibrium:

1. $e(\rho)=1$ for all $\rho \geq \bar{\rho}$,

2. $a(\rho) \leq \bar{a}$ for all $\rho \in[0,1]$.

Proof: Since $\Xi(1, e, a)=1$ for all $e$ and $a$ and $\Xi(\cdot, \cdot, \cdot)$ is continuous, (77) yields $V(\rho)>0$ for all $\rho$ sufficiently close to 1 . But $V(\rho)>0$ implies $e(\rho)=1$.

Next, define $\bar{a}$ implicitly by

$$
\Xi(0,1, \bar{a})=\delta\left(\frac{\Xi(1,1,1)-c}{1-\frac{\delta}{2}}\right)
$$

Observe that $\bar{a}<1$ since $\delta<\frac{2}{3-2 c}$, and $\bar{a}>0$ since the right-hand side of (80) is strictly positive.

Suppose that we can find an equilibrium with $a(\hat{\rho})>\bar{a}$ for $\hat{\rho}>0$. Then, by (77) and Bellman's Principle of Optimality, $\delta V\left(\hat{\rho}^{++}\right) \geq \Xi(\hat{\rho}, e(\hat{\rho}), a(\hat{\rho}))$. Combined with (80), this yields $V\left(\hat{\rho}^{++}\right)>\frac{\Xi(1,1,1)-c}{1-\frac{\delta}{2}}$. Yet, given $\delta<\frac{2}{3-2 c}$, we have

$$
V(1)=\max \left\{\frac{\Xi(1,1,1)-c}{1-\frac{\delta}{2}}, \frac{\frac{1}{2} \Xi(1,1,1)-c}{1-\delta}\right\}=\frac{\Xi(1,1,1)-c}{1-\frac{\delta}{2}},
$$

yielding $V\left(\hat{\rho}^{++}\right)>V(1)$, which cannot be.

In what follows let $\tilde{a}(\cdot)$ denote the unique equilibrium of the auxiliary game analyzed in 
Subsection F.2, $\tilde{V}(\cdot)$ the corresponding value function, and for $\delta<\frac{2}{3-2 c}$ define $\tilde{\rho}$ implicitly by

$$
\begin{cases}\tilde{\rho}=0 & \text { if } \tilde{V}(\rho)>0 \text { for all } \rho>0 \\ \tilde{V}(\tilde{\rho})=0 & \text { otherwise. }\end{cases}
$$

By virtue of Proposition 28, $\tilde{\rho}$ is well defined. Finally, given $\rho>0$, let

$$
\rho^{\dagger}:=\frac{\rho}{\rho+(1-\rho) \tilde{a}(\rho)}
$$

We will now show that there can be at most one equilibrium for $\delta<\frac{2}{3-2 c}$. The proof is somewhat complicated. To help the reader get the gist of the argument, we defer the main result and start with a slightly weaker version of the result, by focusing on the class of equilibria with a non-decreasing value function.

Lemma 17. Let $\delta<\frac{2}{3-2 c}$. In any equilibrium whose value function is non-decreasing:

1. if $\tilde{\rho}=0: e(\rho)=1$ for all $\rho>0$,

2. if $\tilde{\rho}>0$ : $e(\rho)=1$ for all $\rho \geq \tilde{\rho}$ and $e(\rho) \in(0,1)$ for all $\rho \in(0, \tilde{\rho})$.

Proof: We first show the proof of the lemma for the case in which $\tilde{\rho}=0$. The case $\tilde{\rho}>0$ is considered at the end.

Define $\rho_{1}$ implicitly by

$$
\bar{\rho}=\frac{\rho_{1}}{\rho_{1}+\left(1-\rho_{1}\right) \bar{a}},
$$

with $\bar{\rho}$ and $\bar{a}$ satisfying the conditions stated in Lemma 16. Thus $\rho_{1}<\bar{\rho}$ (since $\bar{a}<1$ ), and in any equilibrium $\rho^{++} \geq \bar{\rho}$ for all $\rho \geq \rho_{1}$.

The proof is by induction: we have $e(\rho)=1$ for all $\rho \geq \bar{\rho}$ and we proceed to show that $e(\rho)=1$ for all $\rho \geq \rho_{1}$.

Step 1: for all $\rho \geq \rho_{1}, \Xi(\rho, e(\rho), a(\rho)) \geq \Xi(\rho, 1, \tilde{a}(\rho))$. If $e(\rho)=1$ then $a(\rho)=\tilde{a}(\rho)$ and so the result is trivial. Suppose now that $e(\rho)<1$, and $\Xi(\rho, e(\rho), a(\rho))<\Xi(\rho, 1, \tilde{a}(\rho))$. Then $a(\rho)<\tilde{a}(\rho)$, which in turn implies

$$
\Xi(\rho, e(\rho), a(\rho)) \geq \delta V\left(\rho^{++}\right)=\delta \tilde{V}\left(\rho^{++}\right)>\delta \tilde{V}\left(\rho^{\dagger}\right) \geq \Xi(\rho, 1, \tilde{a}(\rho))
$$

The first inequality follows from $(77)$ and $a(\rho)<1$. The subsequent equality follows from 
noting that $\rho^{++} \geq \bar{\rho}$ while $V(\rho)=\tilde{V}(\rho)$ for all $\rho \geq \bar{\rho}$. The second inequality is due to the fact that since $a(\rho)<\tilde{a}(\rho), \rho^{++}>\rho^{\dagger}$, while $\tilde{V}$ is strictly increasing (Proposition 28). The last inequality follows from $(71)$ and $\tilde{a}(\rho)>0$.

Step 2: for all $\rho \geq \rho_{1}, \tilde{V}\left(\rho^{++}\right) \geq \tilde{V}\left(\rho^{\dagger}\right)$. First, suppose $a(\rho)=0$. Then $\rho^{++}=1$ and, since $\tilde{V}(\cdot)$ is increasing on $(0,1]$ (Proposition 28), $\tilde{V}\left(\rho^{++}\right) \geq \tilde{V}\left(\rho^{\dagger}\right)$.

Next, suppose $\tilde{a}(\rho)=0$. Then $\rho^{\dagger}=1$ and $\Xi(\rho, 1, \tilde{a}(\rho)) \geq \delta \tilde{V}\left(\rho^{\dagger}\right)=\delta \tilde{V}(1)$. Therefore, if we had $\tilde{V}\left(\rho^{++}\right)<\tilde{V}\left(\rho^{\dagger}\right)$ we would have (by Step 1) $\Xi(\rho, e(\rho), a(\rho))>\delta \tilde{V}\left(\rho^{++}\right)$, and since $V(\rho)=\tilde{V}(\rho)$ for all $\rho \geq \bar{\rho}, \Xi(\rho, e(\rho), a(\rho))>\delta V\left(\rho^{++}\right)$. Given $(77)$, this would imply $a(\rho)=0$, and $\rho^{++}=1$. But then $\tilde{V}\left(\rho^{++}\right)=\tilde{V}\left(\rho^{\dagger}\right)$.

Finally, suppose $a(\rho)>0$ and $\tilde{a}(\rho)>0$. We then obtain, in view of (71), (77), Step 1 and $V(\rho)=\tilde{V}(\rho)$ for all $\rho \geq \bar{\rho}$,

$$
\delta \tilde{V}\left(\rho^{++}\right)=\delta V\left(\rho^{++}\right)=\Xi(\rho, e(\rho), a(\rho)) \geq \Xi(\rho, 1, \tilde{a}(\rho))=\delta \tilde{V}\left(\rho^{\dagger}\right) .
$$

Step 3: for all $\rho \geq \rho_{1}, V(\rho) \geq \tilde{V}(\rho)$. Using $V(\rho)=\tilde{V}(\rho)$ for all $\rho \geq \bar{\rho}$, if $V(\cdot)$ is non-decreasing then (77), Step 1 and Step 2 yield

$$
V(\rho) \geq \max \left\{\Xi(\rho, 1, \tilde{a}(\rho))-c+\frac{\delta}{2} V(\rho), \frac{1}{2} \Xi(\rho, 1, \tilde{a}(\rho))-c+\delta\left(\frac{1}{2} V(\rho)+\frac{1}{2} \tilde{V}\left(\rho^{\dagger}\right)\right)\right\},
$$

for all $\rho \geq \rho_{1}$. Comparing (81) with $(71)$ gives $V(\rho) \geq \tilde{V}(\rho)$, for all $\rho \geq \rho_{1}$.

We conclude from Step 3 that $V(\rho)>0$ for all $\rho \geq \rho_{1}$, which in turn implies that $e(\rho)=1$ for all $\rho \geq \rho_{1}$. We can thus repeat Steps 1-3 with $\rho_{1}$ instead of $\bar{\rho}$, and so on. This process defines a sequence $\left\{\rho_{n}\right\}$ such that, for all $n, e(\rho)=1$ for all $\rho \geq \rho_{n}$, and $\rho_{n}=\frac{\rho_{n+1}}{\rho_{n+1}+\left(1-\rho_{n+1}\right) \bar{a}}$. As $\bar{a}<1, \rho_{n} \rightarrow 0$. Thus $e(\rho)=1$ for all $\rho>0$.

We now show the proof of the lemma for the case in which $\tilde{\rho}>0$. Reasoning as in the previous case shows that $V(\rho)>0$ for all $\rho>\tilde{\rho}$, and therefore that $e(\rho)=1$ and $V(\rho)=\tilde{V}(\rho)$ for all $\rho>\tilde{\rho}$. Furthermore $V(1) \geq \frac{\frac{1}{2} \Xi(1,1,1)-c}{1-\delta}>0$, from which follows that $e(\rho)>0$ for all $\rho>0$; if this were not the case, acquiring information would yield the strategic agency $V(1)>0$. Hence, we are only left to show that $e(\tilde{\rho})=1$. Suppose that $e(\tilde{\rho})<1$. Then 
$\tilde{\rho}^{+}>\tilde{\rho}$, and so $\tilde{V}\left(\tilde{\rho}^{+}\right)>0$. Hence, we obtain

$$
\begin{aligned}
V(\tilde{\rho}) & \geq \frac{1}{2} \Xi(\tilde{\rho}, e(\tilde{\rho}), a(\tilde{\rho}))-c+\delta\left(\frac{1}{2} V\left(\tilde{\rho}^{+}\right)+\frac{1}{2} V\left(\tilde{\rho}^{++}\right)\right) \\
& =\frac{1}{2} \Xi\left(\tilde{\rho}^{+}, 1, a(\tilde{\rho})\right)-c+\delta\left(\frac{1}{2} V\left(\tilde{\rho}^{+}\right)+\frac{1}{2} V\left(\tilde{\rho}^{++}\right)\right) \\
& =\frac{1}{2} \Xi\left(\tilde{\rho}^{+}, 1, \tilde{a}\left(\tilde{\rho}^{+}\right)\right)-c+\delta\left(\frac{1}{2} \tilde{V}\left(\tilde{\rho}^{+}\right)+\frac{1}{2} \tilde{V}\left(\tilde{\rho}^{++}\right)\right) \\
& =\tilde{V}\left(\tilde{\rho}^{+}\right)>0 .
\end{aligned}
$$

The first line follows from (77), and the second from noting that $\Xi(\tilde{\rho}, e(\tilde{\rho}), a(\tilde{\rho}))=\Xi\left(\tilde{\rho}^{+}, 1, \tilde{a}(\tilde{\rho})\right)$. The third line is obtained by noting that, since $e\left(\tilde{\rho}^{+}\right)=1=e\left(\tilde{\rho}^{++}\right)$, the trade off between lying and telling the truth faced by the strategic agency with reputation $\tilde{\rho}$ is the same as the trade off faced by the strategic agency with reputation $\tilde{\rho}^{+}$in the auxiliary game. Therefore, $a(\tilde{\rho})=\tilde{a}\left(\tilde{\rho}^{+}\right)$. The sequence above yields $V(\tilde{\rho})>0$, and so $e(\tilde{\rho})=1$.

Lemma 18. Let $\delta<\frac{2}{3-2 c}$. In any equilibrium:

1. if $\tilde{\rho}=0: e(\rho)=1$ for all $\rho>0$,

2. if $\tilde{\rho}>0$ : $e(\rho)=1$ for all $\rho \geq \tilde{\rho}$ and $e(\rho) \in(0,1)$ for all $\rho \in(0, \tilde{\rho})$.

Proof: We will show the proof of the lemma for the case in which $\tilde{\rho}=0$. We omit the proof of the case $\tilde{\rho}>0$, which is very similar to the case we consider.

First, notice that $V(1) \geq \frac{\frac{1}{2}-c}{1-\delta}>0$. It ensues that $e(\rho)>0$ for all $\rho>0$; if this were not the case, acquiring information would yield the strategic agency $V(1)>0$. Since $a(\rho)<1$ for all $\rho>0$ (Lemma 16) Bellman's Principle of Optimality yields

$$
V(\rho)=\Xi(\rho, e(\rho), a(\rho))-c+\frac{\delta}{2} V\left(\rho^{+}\right)
$$

Define $\rho_{1}$ implicitly by

$$
\bar{\rho}=\frac{\rho_{1}}{\rho_{1}+\left(1-\rho_{1}\right) \bar{a}}
$$

with $\bar{\rho}$ and $\bar{a}$ satisfying the conditions stated in Lemma 16. Thus $\rho_{1}<\bar{\rho}$ (since $\bar{a}<1$ ), and in any equilibrium $\rho^{++} \geq \bar{\rho}$ for all $\rho \geq \rho_{1}$.

The proof is by induction: we have $e(\rho)=1$ for all $\rho \geq \bar{\rho}$ and we proceed to show that $e(\rho)=1$ for all $\rho \geq \rho_{1}$. 
Suppose that we can find $\rho \geq \rho_{1}$ such that $e(\rho)<1$. We claim that $e(\rho)<1$ implies $e\left(\rho^{+}\right)<1$. To see this, observe that if $e\left(\rho^{+}\right)=1$ then

$$
\begin{aligned}
V(\rho) & \geq \frac{1}{2} \Xi(\rho, e(\rho), a(\rho))-c+\delta\left(\frac{1}{2} V\left(\rho^{+}\right)+\frac{1}{2} V\left(\rho^{++}\right)\right) \\
& =\frac{1}{2} \Xi\left(\rho^{+}, 1, a(\rho)\right)-c+\delta\left(\frac{1}{2} V\left(\rho^{+}\right)+\frac{1}{2} V\left(\rho^{++}\right)\right) \\
& =\frac{1}{2} \Xi\left(\rho^{+}, 1, \tilde{a}\left(\rho^{+}\right)\right)-c+\delta\left(\frac{1}{2} \tilde{V}\left(\rho^{+}\right)+\frac{1}{2} \tilde{V}\left(\rho^{++}\right)\right) \\
& =\tilde{V}\left(\rho^{+}\right) .
\end{aligned}
$$

The first line follows from (77), and the second from noting that $\Xi(\rho, e(\rho), a(\rho))=\Xi\left(\rho^{+}, 1, a(\rho)\right)$. The third line is obtained by noting that, since $e\left(\rho^{+}\right)=1=e\left(\rho^{++}\right)$, the trade-off between lying and telling the truth faced by the strategic agency with reputation $\rho$ is the same as the trade-off faced by the strategic agency with reputation $\rho^{+}$in the auxiliary game. Therefore, $a(\rho)=\tilde{a}\left(\rho^{+}\right)$. The sequence (83) yields $V(\rho) \geq \tilde{V}\left(\rho^{+}\right)>0$, and so $e(\rho)=1$, contradicting our initial assumption.

Since we showed that $e(\rho)<1$ implies $e\left(\rho^{+}\right)<1$, if we can find $\rho \geq \rho_{1}$ such that $e(\rho)<1$ then there exists a strictly increasing sequence $\left\{\rho_{n}\right\}$ with $e\left(\rho_{n}\right)<1$ for all $n$, and $\rho_{n+1}=\rho_{n}^{+}$. Let $\hat{\rho}=\lim _{n \rightarrow \infty} \rho_{n}$; by Lemma 16, $\hat{\rho}<\bar{\rho}+z$ for all $z>0$, as otherwise for $n$ large enough we would get $e\left(\rho_{n}\right)=1$. As $\bar{\rho}<1$ this is turn implies that $\lim _{n \rightarrow \infty} e\left(\rho_{n}\right)=1$.

We next claim that for all $\epsilon>0$ there exists $N$ such that, for all $n>N$ :

$$
\left|\Xi\left(\rho_{n}, e\left(\rho_{n}\right), a\left(\rho_{n}\right)\right)-\Xi(\hat{\rho}, 1, \tilde{a}(\hat{\rho}))\right|<\epsilon \text {. }
$$

To see this, suppose that $\tilde{a}(\hat{\rho})>0$ (the case $\tilde{a}(\hat{\rho})=0$ can be dealt with in a similar way). Hence,

$$
\delta \tilde{V}\left(\hat{\rho}^{\dagger}\right)=\Xi(\hat{\rho}, 1, \tilde{a}(\hat{\rho}))
$$

We therefore have

$$
\lim _{n \rightarrow \infty} \Xi\left(\rho_{n}, e\left(\rho_{n}\right), 0\right)=\Xi(\hat{\rho}, 1,0)<\Xi(\hat{\rho}, 1, \tilde{a}(\hat{\rho}))=\delta \tilde{V}\left(\hat{\rho}^{\dagger}\right) \leq \delta \tilde{V}(1),
$$

from which follows that, for $n$ large enough, $a(\rho)>0$. Hence $\delta V\left(\rho_{n}^{++}\right)=\Xi\left(\rho_{n}, e\left(\rho_{n}\right), a\left(\rho_{n}\right)\right)$ for all $n$ large enough. Since, $\rho_{n}^{++} \geq \bar{\rho}$ for all $n$, we obtain

$$
\delta \tilde{V}\left(\rho_{n}^{++}\right)=\Xi\left(\rho_{n}, e\left(\rho_{n}\right), a\left(\rho_{n}\right)\right),
$$


for all $n$ large enough. That (84) holds for sufficiently large $n$ now follows from (85), (86) and continuity of $\tilde{V}(\cdot)$ (see Proposition 28).

Now, by construction of $\left\{\rho_{n}\right\}$, (82) yields

$$
V\left(\rho_{n}\right)=\sum_{k=0}^{\infty}\left(\frac{\delta}{2}\right)^{k}\left(\Xi\left(\rho_{n+k}, e\left(\rho_{n+k}\right), a\left(\rho_{n+k}\right)\right)-c\right)
$$

for all $n$. Moreover, we showed above that for all $\epsilon>0$ there exists $N$ such that, for all $n>N$, (84) holds. Thus, for all $\eta>0$, choosing $\epsilon$ sufficiently small and $n$ sufficiently large gives

$$
\left|V\left(\rho_{n}\right)-\sum_{k=0}^{\infty}\left(\frac{\delta}{2}\right)^{k}(\Xi(\hat{\rho}, 1, \tilde{a}(\hat{\rho}))-c)\right|<\eta,
$$

i.e., noting that $\sum_{k=0}^{\infty}\left(\frac{\delta}{2}\right)^{k}(\Xi(\hat{\rho}, 1, \tilde{a}(\hat{\rho}))-c)=\frac{\Xi(\hat{\rho}, 1, \tilde{a}(\hat{\rho}))-c}{1-\frac{\delta}{2}}=\tilde{V}(\hat{\rho})$,

$$
\left|V\left(\rho_{n}\right)-\tilde{V}(\hat{\rho})\right|<\eta
$$

As $\tilde{V}(\hat{\rho})>0$, we conclude that $V\left(\rho_{n}\right)>0$, implying $e\left(\rho_{n}\right)=1$. This contradicts the construction of the sequence $\left\{\rho_{n}\right\}$. Thus $e(\rho)=1$ for all $\rho \geq \rho_{1}$.

We can now repeat the steps above with $\rho_{1}$ instead of $\bar{\rho}$, and so on. This process defines a sequence $\left\{\hat{\hat{\rho}}_{n}\right\}$ such that, for all $n, e(\rho)=1$ for all $\rho \geq \hat{\hat{\rho}}_{n}$, and $\hat{\hat{\rho}}_{n}=\frac{\hat{\hat{\rho}}_{n+1}}{\hat{\hat{\rho}}_{n+1}+\left(1-\hat{\hat{\rho}}_{n+1}\right) \bar{a}}$. As $\bar{a}<1$, $\hat{\hat{\rho}}_{n} \rightarrow 0$. Thus $e(\rho)=1$ for all $\rho>0$.

Proposition 30. Let $\delta<\frac{2}{3-2 c}$. There exists a unique equilibrium. This equilibrium is such that, for some cutoff $\rho_{c}$ :

1. $e(0)=a(0)=0$;

2. $e(\rho)=1$ for all $\rho \in(\tilde{\rho}, 1]$, and $e(\rho) \in(0,1)$ for all $\rho \in(0, \tilde{\rho})$;

3. $a(\rho)=0$ for all $\rho \in\left[\rho_{c}, 1\right]$, and $a(\rho)>0$ for all $\rho \in\left(0, \rho_{c}\right)$.

Proof: We take up the case in which $\tilde{\rho}>0$. The case in which $\tilde{\rho}=0$ is similar and simpler, we therefore omit the proof. 
By virtue of the one-shot deviation principle, the following strategy is an equilibrium:

$$
\begin{cases}e(0)=a(0)=0 ; & \text { for all } \rho \geq \tilde{\rho} \\ e(\rho)=1 & \text { for all } \rho<\tilde{\rho} ; \\ \tilde{\rho}=\frac{\rho}{\rho+(1-\rho) e(\rho)} & \text { for all } \rho \geq \tilde{\rho} ; \\ a(\rho)=\tilde{a}(\rho) & \text { for all } \rho \in(0, \tilde{\rho}) . \\ a(\rho)=\tilde{a}(\tilde{\rho}) & \end{cases}
$$

Uniqueness follows from Lemma 18. The existence of the cutoff $\rho_{c}$ is a consequence of Proposition 28.

Proposition 31. Let $\delta \neq \frac{2}{3-2 c}$. There is a unique equilibrium with observable information acquisition and contingent fees. In this equilibrium, if $\delta>\frac{2}{3-2 c}$, then $e(\rho)=a(\rho)=1$ for all $\rho>0$. If instead $\delta<\frac{2}{3-2 c}$ then the equilibrium is characterized by cutoffs $\rho_{c_{1}}<1$ and $\rho_{c_{2}}<1$ such that:

1. $e(\rho)=1$ if $\rho>\rho_{c_{1}}$ and $e(\rho) \in(0,1)$ if $\rho \in\left(0, \rho_{c_{1}}\right)$;

2. $a(\rho)=0$ if $\rho>\rho_{c_{2}}$ and $a(\rho)>0$ if $\rho \in\left(0, \rho_{c_{2}}\right)$.

Furthermore, $e(0)=a(0)=0$ for all $\delta$.

Proof: Follows from Propositions 29 and 30.

Proposition 32. There is a unique equilibrium with observable information acquisition and upfront fees. In this equilibrium, $e(\rho)=a(\rho)=1$ for all $\rho>0$.

Proof: Consider an equilibrium of the game with observable information acquisition and fees received upfront by the agency such that $e(0)=a(0)=0$. Let $V$ denote the value function. Thus $V(0)=0$, while $V(1) \geq \frac{\frac{1}{2} \Xi(1,1,1)-c}{1-\delta}>0$. Hence $e(1)=1$. It immediately follows that $e(\rho)>0$ for all $\rho>0$; if this were not the case, acquiring information would yield the strategic 
agency $V(1)>0$. This gives

$$
\begin{aligned}
& V(\rho)= \\
& \max \left\{\mathbb{P}\left(r_{t}=1 \mid \rho, e(\rho), a(\rho)\right) \Xi(\rho, e(\rho), a(\rho))-c+\frac{\delta}{2} V\left(\rho^{+}\right),\right. \\
& \left.\quad \mathbb{P}\left(r_{t}=1 \mid \rho, e(\rho), a(\rho)\right) \Xi(\rho, e(\rho), a(\rho))-c+\delta\left(\frac{1}{2} V\left(\rho^{+}\right)+\frac{1}{2} V\left(\rho^{++}\right)\right)\right\},
\end{aligned}
$$

for all $\rho>0$. Moreover, no matter $e(\rho)$, we have $\mathbb{P}\left(r_{t}=1 \mid \rho, e(\rho), 1\right) \Xi(\rho, e(\rho), 1)=1$. This implies that we can find $\bar{a}<1$ such that, either (i) $V(\rho)>0$ or (ii) $a(\rho)<\bar{a}$ and $V\left(\rho^{++}\right)=0$. Case (ii) is however impossible as it implies the existence of a sequence $\left\{\rho_{n}\right\}$ tending to 1 as $n \rightarrow \infty$ and such that $V\left(\rho_{n}\right)=0$ for all $n$. Therefore, $V(\rho)>0$ for all $\rho>0$. It ensues that $e(\rho)=a(\rho)=1$ for all $\rho>0$.

Proof of Proposition 27: Follows from Propositions 31 and 32. 


\section{Online Appendix G}

In this appendix we characterize the set of socially optimal fee structures. Consider a fee structure such that: if $\rho_{t}=\rho_{1}$ then $\phi_{t}(1)=a$ and $\phi_{t}(-1)=b$ while if $\rho_{t}=0$ then $\phi_{t}(1)=y$ and $\phi_{t}(-1)=z$. The fee structure is socially optimal if: firms prefer to get a rating as long as $\rho_{t}=\rho_{1}$ and their beliefs satisfy $\hat{e}_{t}=1$ (call this condition 1 ) and there exist an equilibrium of the game with such a fee structure in which $e\left(\rho_{1}\right)=1$ (call this condition 2). Condition 1 boils down to:

$$
\frac{a}{2}+\frac{b}{2} \leq\left(\frac{1}{2} \cdot 1+\frac{1}{2} \cdot 0\right)
$$

where the left-hand side is the firm's expected payment to the agency and the right-hand side is the firm's expected revenue from investors if firm $t$ decides to obtain a rating for $\rho_{t}=\rho_{1}$ and $\hat{e}_{t}=1$. Condition 1 is equivalent to:

$$
a+b \leq 1
$$

Condition 2 boils down to:

$$
\frac{a-b}{2}+c \leq \frac{\delta}{2}\left(\cdot \frac{1}{1-\delta}\left(\frac{a+b}{2}-c\right)-\max \left\{\frac{y}{1-\delta}, \frac{y+2}{2(1-\delta)}-c\right\}\right)
$$

where the left-hand side the CRA's short-run incentive to shirk and the right-hand side is the CRA's long-run incentive to acquire information for $\rho_{t}=\rho_{1}$ and $\hat{e}_{t}=1$. Rearranging terms, this condition can be written as:

$$
\frac{b-a}{2}+\frac{a \delta}{2-\delta} \geq c+\max \left\{\frac{y}{1-\delta}, \frac{y+2}{2(1-\delta)}-c\right\} \frac{2(1-\delta)}{2-\delta} .
$$

The largest set of $a$ and $b$ for which this condition holds is obtained for $y=z=0$, in which case the condition reduces to:

$$
\frac{b-a}{2}+\frac{a \delta}{2-\delta} \geq c
$$

